\title{
A spatial map in the somatosensory cortex
}

2 Xiaoyang Long and Sheng-Jia Zhang*

3 Department of Neurosurgery, Xinqiao Hospital, Army Medical University,

4 Chongqing, China

$5 \quad *$ To whom correspondence should be addressed.

6 Correspondence and requests for materials should be addressed to:

7 sheng-jia.zhang@tmmu.edu.cn or sheng-jia.zhang@outlook.com 


\section{Abstract}

9 Spatially selective firing in the forms of place cells, grid cells, boundary vector/border cells and head direction cells are the basic building blocks of a canonical spatial navigation system centered on the hippocampal-entorhinal complex. While head direction cells can be found throughout the brain, spatial tuning outside the hippocampal formation are often non-specific or conjunctive to other representations such as a reward. Although the precise mechanism of spatially selective activities is not understood, various studies show sensory inputs (particularly vision) heavily modulate spatial representation in the hippocampal-entorhinal circuit. To better understand the contribution from other sensory inputs in shaping spatial representation in the brain, we recorded from the primary somatosensory cortex in foraging rats. To our surprise, we were able to identify the full complement of spatial activity patterns reported in the hippocampal-entorhinal network, namely, place cells, head direction cells, boundary vector/border cells, grid cells and conjunctive cells. These newly identified somatosensory spatial cell types form a spatial map outside the hippocampal formation and support the hypothesis that location information is necessary for body representation in the somatosensory cortex, and may be analogous to spatially tuned representations in the motor cortex relating to the movement of body parts. Our findings are transformative in our understanding of how spatial information is used and utilized in the brain, as well as functional operations of the somatosensory cortex in the context of rehabilitation with brain-machine interfaces. 


\section{Introduction}

Spatial representation in the brain has been largely associated with the hippocampus and the entorhinal cortex ${ }^{1,2}$. Place cells ${ }^{3}$ were originally discovered in the hippocampus, with boundary vector/border cells ${ }^{4,5}$, grid cells ${ }^{6}$ and conjunctive cells ${ }^{7}$ discovered later in the entorhinal cortex. Unlike the location-specific spatial cells in the hippocampal-entorhinal circuit, head direction cells ${ }^{8}$ can be found in areas ranging from the diencephalon to thalamic, striatal and cortical regions 9 .

Despite the focus on hippocampal-entorhinal contributions to spatial representation, location-dependent firing conjunctive to other sensory/cognitive (e.g. reward) information has been reported in sensory areas ${ }^{10-12}$ and cortical regions heavily interconnected with the hippocampal-entorhinal system such as the septum, subicular complex, cingulate cortex, parietal cortex and retrosplenial cortex ${ }^{13-20}$. In rodents, the only extra-parahippocampal region exhibiting apparent location-dependent firing restricted to space alone is the anterior claustrum, where stable, visually-anchored place cell- and border cell-like activities can be recorded ${ }^{21}$. A preliminary report also suggests the dorsal geniculate nucleus may contain place cells similar to those found in the hippocampus ${ }^{22}$. In humans, hexadirectional grid-like signals have been observed in many brain regions outside the hippocampal formation including the posterior cingulate cortex, the medial prefrontal cortex, the retrosplenial cortex, the medial parietal cortex and the frontal cortex ${ }^{23-26}$ while grid-cell-like neuronal representations were identified with intracranial electroencephalography recordings on presurgical epilepsy patients and fMRI studies in the human entorhinal cortex ${ }^{27-29}$. Computational modelling also predicted the existence of grid cell-like neurons in all cortical regions throughout the neocortex ${ }^{30}$. These results therefore indicate that spatial tuning is much more distributed into multiple cortical domains beyond the classical hippocampal formation than we previously thought.

The exact physiological mechanism of spatial selectivity in the hippocampal-entorhinal 
system is still incompletely understood. Lesions to either area severely affect, but do not abolish spatially selective activities in the other ${ }^{31,32}$. Past studies have shown manipulation of visual cues ${ }^{33,34}$, olfaction ${ }^{35}$ and vibrissae inputs ${ }^{36}$ all impact on hippocampal place cell activity, yet none is necessary. Recent theoretical work has suggested the somatosensory area may contain higher-order presentations of the body itself beyond elementary sensory information, of which location presentation is integral in a "body simulation" model of somatosensation ${ }^{37}$. While there are place cell-like activities in the wheelchair-seated monkey sensorimotor cortices ${ }^{38}$, it is unclear if these spatial representations are comparable to their freely moving rodent equivalents. Based on the assumption that somatosensation should play a major role in sensory inputs shaping parahippocampal spatial activity 39,40 and spatial presentation has been theorized to be crucial for body representation ${ }^{37}$, we sought to identify spatially selective activities in the primary somatosensory cortex (S1). Remarkably, we were able to detect all spatial cell types reported in the hippocampal-entorhinal system ${ }^{1,2}$ within the S1. We show these spatial cell types within this newly discovered somatosensory spatial representation system share similar properties to their parahippocampal counterparts in terms of stability and response to environmental manipulations ${ }^{41}$.

\section{Results}

\section{Extracellular recording from the somatosensory cortex}

We obtained neurophysiological recordings in freely behaving rats by using tetrodes mounted on movable microdrives. We recorded 2025 putative single units from eight implanted rats across 287 recording sessions. Recordings were obtained mainly from layers IV to VI within the S1 area (Fig. 1a and Fig. S1), as confirmed by the histological reconstruction. The tetrodes were generally lowered in steps of either $25 \mu \mathrm{m}$ or 50 $\mu \mathrm{m}$ per day. The recording sessions lasted between 10 to 30 minutes to ensure sufficient coverage of the whole arena, which was facilitated by foraging food pellets intermittently thrown into the arena. Spike sorting was performed manually offline 
using TINT (Axona, St. Albans, U.K.). The separation of the clusters and waveforms was quantitatively measured with an isolation distance and the L-ratio ${ }^{42}$ (Fig. S2).

We applied the same analysis described in previous work ${ }^{7,17,43}$ on 2025 somatosensory cells and 392 met the criteria for various types (e.g. place, head direction, boundary vector/border and grid cells) of spatial tuning (Fig. S3). The number of each functionally distinct somatosensory spatial cell type from individual implanted animals was summarized in the supplementary Table S1. No significant differences were observed among spatial information, mean vector length and grid score across different animals (ANOVA test, $P=0.38,0.08$ and 0.18 , respectively, Figs. S4a, b and d). Both border score and grid spacing remained similar across interindividual animals except that they are slightly different in one of eight animals (ANOVA test, $* P<0.05$, Figs. S4c-d). The distribution of different spatial cell types across different animals suggested, at least between layers IV and VI, there was a tendency for head direction cells to avoid layer VI and the presence of spatially tuned somatosensory cells was sparse in superficial layer V (Figs. S3b-f). Place cells appeared to predominate deeper layers (Figs. S3a-c).

\section{Place cells in the somatosensory cortex}

As illustrated in Fig. 1, somatosensory cells possessing place cell-like properties were the most abundant spatial cell type encountered (Figs. S3a-c). Spatially selective firing patterns similar to those originally described for hippocampal place cells ${ }^{3}$ can be observed (Fig. 1b and Fig. S5). These S1 place cells were classified if their spatial information (SI) score ${ }^{44}$ exceeds a stringent $99^{\text {th }}$ percentile threshold (SI $>1.50$ cut-off) from the population-based distribution of shuffled rate maps (Fig. 1d). 195 out of the 2025 S1 neurons $(9.63 \%)$ met criterion and thus are referred to as "place cells" from here on. These place cells also passed the spatial threshold for the within-cell shuffling (Fig. 1c and Fig. S6a), which is less stringent than the population shuffling. The withincell shuffling outcome is also true of other types of somatosensory spatial cells (Figs.

S6b-d). Accordingly, we used the population shuffling for defining all different types 
111 of somatosensory spatial cells throughout this study. Furthermore, we applied the maximum-likelihood approach ${ }^{45,46}$ to evaluate inhomogeneous sampling biases and

113 found that somatosensory place cells showed a robust locational effect, which was not altered by the correction algorithm (Figs. S7a-c). The average SI in bits per spike of all 195 classified S1 place cells was $2.19 \pm 0.04$ (mean \pm s.e.m., all data are shown as mean \pm s.e.m. unless otherwise indicated). This percentage was significantly higher than expected by random selection from the entire shuffled population (Fig. 1 d; $Z=39.03$, $P<0.001$; binomial test with expected $P_{0}$ of 0.01 among large samples).

We noted that if a $95^{\text {th }}$ percentile criterion was applied, 511 out of the $2025 \mathrm{~S} 1$ neurons $(24.00 \%)$ would be classified as place cells (average SI of $1.59 \pm 0.03$; cut-off $=.81$ ). This percentage was substantially higher than expected with a random selection from the entire shuffled population $($ Fig. $1 d ; Z=109.61, P<0.001$; binomial test with expected $P_{0}$ of 0.01 among large samples). We found that the percentage of identified $\mathrm{S} 1$ place cells is lower within the somatosensory cortex than that of hippocampal place cells ${ }^{47}$. The average peak firing rate and the mean firing rate of identified somatosensory location-specific place cells were $10.32 \pm 0.37 \mathrm{~Hz}$ and $0.70 \pm 0.04 \mathrm{~Hz}$ (Fig. S8a), respectively. The histograms of peak-to-peak amplitude and peak-to-trough spike width were shown in Fig. S8e. Like a mixture of a single place field and multiple place fields identified from the hippocampal place cells ${ }^{48}$, we found that the proportion of multi-field place cells was $25.64 \%$. The average somatosensory spatial firing field size was $891.82 \pm 32.65 \mathrm{~cm}^{2}$ (Fig. 1f). To quantify the smoothness of recorded somatosensory place fields, we calculated the spatial coherence by computing the mean correlation between the firing rate of each bin and the averaged firing rate of the 8 neighboring bins from unsmoothed spatial firing rate maps ${ }^{34}$. For 195 recorded somatosensory place cells, the average spatial coherence was $0.61 \pm 0.01$ (Fig. 1g). Furthermore, we also calculated spatial sparsity to evaluate the extent of spatial information in each spike discharged by $\mathrm{S} 1$ place cells ${ }^{44}$. The spatial sparsity measure of S1 place cells was $0.12 \pm 0.01$ (Fig. 1h). Besides, S1 place cells can be found at any position within the arena for the uniformity testing based on the Friedman-Rafsky's 
MST test (Fig. 1e, Friedman-Rafsky's MST test, $P=0.42$, two-tailed $z$-test) as in the hippocampus ${ }^{49}$.

Place cell activity was defined not only by its location-specific firing but also by the stability of its spatial representation. To this end, we next evaluated the spatial stability of somatosensory place fields by computing the spatial correlations between single intra-trial behavioral sessions. The average spatial correlation for S1 place cells was $0.64 \pm 0.01$ (Fig. S9d) between the two halves of a recording session (Figs. S9a-c), which remain stable in extended recording sessions up to $30 \mathrm{~min}$ (Figs. S10a-c). Hippocampal place cell activities were known to be heavily modulated by visual cues ${ }^{33}, 34$, and were known to rotate with the positioning of visual cues ${ }^{34}$. We performed similar experiments to test if S1 place cell activities were modulated by visual cues by moving it $90^{\circ}$ from its original position and back. Remarkably, the relative position of S1 place fields did indeed rotate with the placement of the visual cue and remained stable after the cue was moved back to its original position (Figs. S11a-c). Furthermore, as hippocampal place codes were reorganized to remap under different geometric environments ${ }^{50,51}$, we also found that $\mathrm{S} 1$ place fields remapped between a square box and a circular box in the same recording room with changed firing rate and firing location (Figs. S12a-c).

To measure the animal's running speed within and outside the firing fields of S1 place cells, we calculated the average "in-field" and "out-field" running speed $(>2.5 \mathrm{~cm} / \mathrm{s})$ for 195 identified place cells in S1. We quantified the average running speed across all the spatial bins within the firing field of the place cell ("in-field" running speed) as well as outside the firing field of the place cell ("out-field" running speed) (Fig. S13a). We found that there was no significant difference between the average "in-field" and "outfield" running speed $(14.58 \pm 0.25 \mathrm{~cm} / \mathrm{s}$ vs $15.00 \pm 0.18 \mathrm{~cm} / \mathrm{s}, n=195, P=0.10$, twotailed paired $t$-test, Fig. S13b). To evaluate the effect of the animal's running speed on spatial responses, we calculated the spatial information scores of S1 place cells for running speed above $2.5 \mathrm{~cm} / \mathrm{s}$ and below $2.5 \mathrm{~cm} / \mathrm{s}$, respectively. We found that the place 
fields during inactive mobility are less prevalent with lower spatial information score border cells (Figs. S27a-c) and S1 grid cells (Figs. S30a-c). As for S1 head direction cells, it appeared that head directional responses were less influenced with immobility than other three S1 spatial cell types (Figs. S21a-c).

a

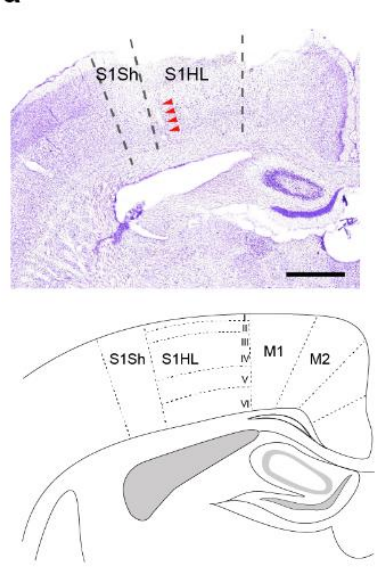

b

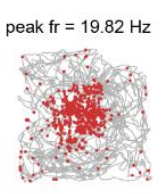

peak $\mathrm{fr}=21.43 \mathrm{~Hz}$
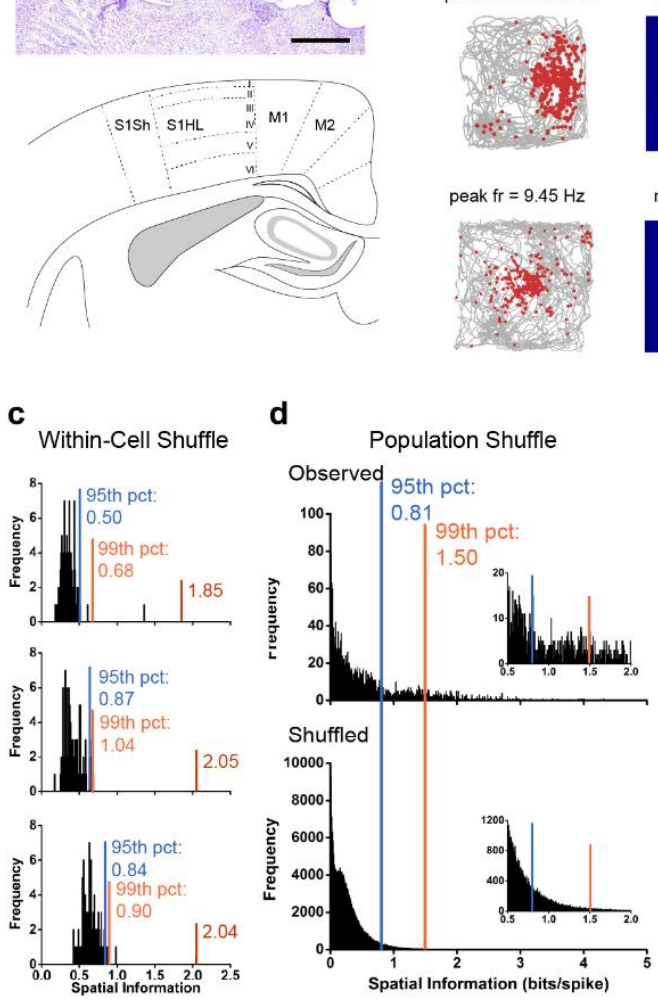

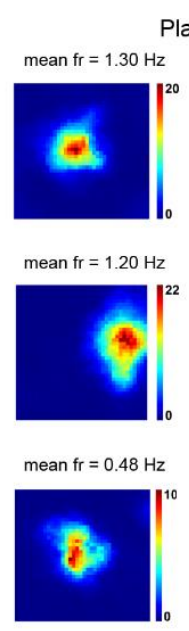

e

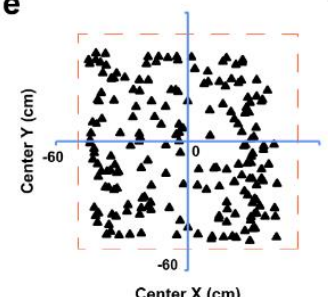

g

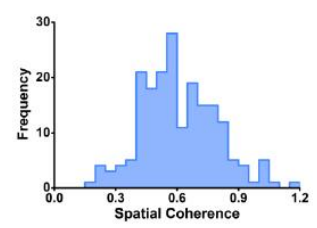

Place Cells
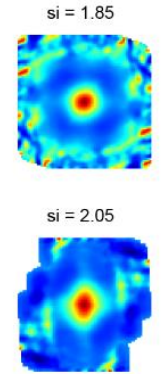

si $=2.04$

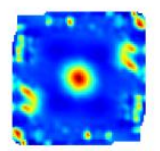

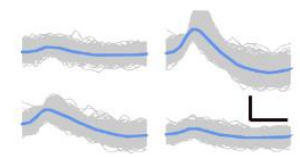
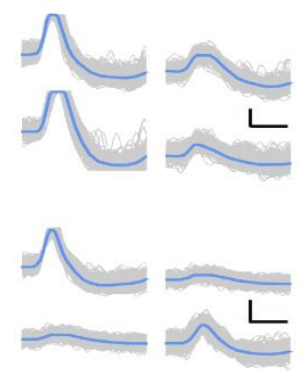

$\mathbf{f}$

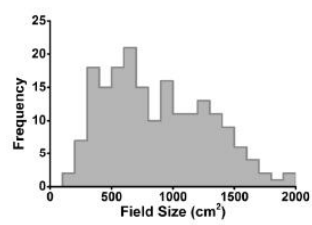

h

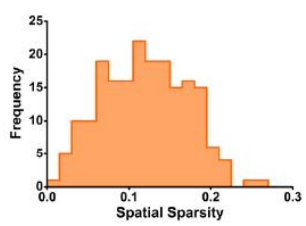

Fig. 1 Place cells in the somatosensory cortex.

(a) A Nissl-stained coronal section (upper) showing tetrode tracking trajectory (arrowheads) through all of six layers across the rat primary somatosensory cortex. Broken dashes depict the boundaries of the hindlimb (S1HL) and shoulder (S1Sh) regions of the primary somatosensory cortex (S1). The bottom panel shows the schematic delimitation of the different layers and sub-regions of the primary somatosensory cortex. Scale bar, $1 \mathrm{~mm}$. (b) Trajectory (grey line) with superimposed spike locations (red dots) (left column); heat maps of spatial firing rate (middle left 
column) and autocorrelation (middle right column) are color-coded with dark blue indicating minimal firing rate and dark red indicating maximal firing rate. The scale of the autocorrelation maps is twice that of the spatial firing rate maps. Peak firing rate (fr), mean firing rate (fr) and spatial information (si) for each representative cell are labelled at the top of the panels. Spike waveforms on four electrodes are shown on the right column. Scale bar, $150 \mu \mathrm{V}, 300 \mu \mathrm{s}$. (c) Distribution of within-cell shuffled spatial information for three representative somatosensory place cells. The orange and blue stippled lines mark the $99^{\text {th }}$ and the $95^{\text {th }}$ percentile significance level of each randomly shuffled distribution, respectively. The red line indicates the observed spatial information. (d) Distribution of spatial information for all isolated somatosensory units. The top panel shows the distribution for observed spatial information. The bottom panel shows the distribution for randomly shuffled data from the same sample. The orange and blue stippled lines mark the $99^{\text {th }}$ and the $95^{\text {th }}$ percentile significance level of each randomly shuffled distribution, respectively. A zoomed panel shows the magnification of the specified area marked by the red dashed rectangle. (e) The uniformly areal distribution of various place firing fields relative to the field center. (f-h) The population histograms of place firing field sizes (f), spatial coherence (g) and spatial sparsity (h) for all of 195 identified somatosensory place cells.

\section{Head direction cells in the somatosensory cortex}

Head direction cells discharge only when the animal's head is at a particular angle respective to the environment ${ }^{8}$. To classify the preferred firing angle of each cell, the mean vector length was used to compute head direction tuning ${ }^{7}$. Cells were categorized as head direction cells if the mean vector length exceeded the $99^{\text {th }}$ percentile of the total distribution of the shuffling procedure from the entire pool of putative single cells (Fig. 2c). 80 cells $(3.95 \%$ of all recorded cells) met the criterion, showing significant modulation with the animal's head direction (Fig. 2a and Fig. S15), with the average mean vector length found to be $0.53 \pm 0.01$ and the mean firing rate to be $0.96 \pm 0.11$

(Fig. S8b). The histograms of peak-to-peak amplitude and peak-to-trough spike width were displayed in Fig. S8f. Besides, the within-cell shuffling for those three 
representative somatosensory head direction cells generated similar results to the population shuffling (Fig. 2b). This percentage is significantly higher than expected by random selection from the entire shuffled population (Fig. 2c; $Z=13.34, P<0.001$; binomial test with expected $P_{0}$ of 0.01 among large samples). Notably, 352/2025 identified S1 neurons $(17.38 \%)$ fulfilled the lower $95^{\text {th }}$ percentile criterion with the threshold of mean vector length being 0.26. To correct for the possible effects of direction and location for identified head direction cells, we applied the maximumlikelihood correction approach ${ }^{45,46}$ and found that the somatosensory head directional responses remain unaltered from the corrected directional firing rate histograms (Figs. S16a-c).

To measure the sharpness of the head directional tuning curve, we quantified the average full width at half maximum (FWHM) of somatosensory head direction cells. The average FWHM of the angular distributions was $77.58 \pm 4.36$ degrees (mean \pm s.e.m.) (Fig. 2d). The distribution of head direction preferences was uniformly distributed across $360^{\circ}$ (Rayleigh test for uniformity: $P=0.17$, Fig. 2e). We next evaluated the intra-trial directional stability by correlating the distributed firing rate across all directional bins between the first and second halves of individual recording trials. We found head direction related firing to be stable within sessions (Figs. S17ac), with the average angular correlation coefficient for S1 head direction cells being $0.58 \pm 0.02$ (Fig. S17d). Like hippocampal place cells, head direction cells also anchored to salient visual cues ${ }^{52}$. We further tested the specificity and stability of S1 head direction cells by rotating a salient visual cue $90^{\circ}$ from its original position in the enclosure and back (Fig. S18, as in S1 place cells described above). As with S1 place cells, S1 head direction cells also rotated with the movement of the salient visual cue (Figs. S18a-c).

To measure the difference of the animal's angular velocity within and outside preferred 237 firing directions for those identified head direction cells in S1, we calculated the average angular velocity in the "preferred firing directions" and "non-preferred firing 
239 directions" for 80 identified head direction cells in S1 (Fig. S19a). We found that there

240 was no significant difference between the average angular velocity in the "preferred

241 firing directions" and "non-preferred firing directions" for head direction cells in S1

$242 \quad(52.97 \pm 1.15$ degrees/s vs $55.52 \pm 1.34$ degrees $/ \mathrm{s}, n=80, P=0.12$, two-tailed paired $t$ -

243 test, Fig. S19b).

244 Although the previous study had shown that food chasing had little effect on head

245 directional firing in the rat anterior thalamic nuclei ${ }^{53}$, we evaluated the firing patterns

246 of head direction cells in the S1 without food pellet dispersion. We recorded the same

247 head direction cell in two consecutive secessions with (bottom panels in Fig. S20a) and

248 without (upper panels in Fig. S20a) food pellets, respectively. No significant

249 differences were observed to suggest that food chasing did not modulate preferred firing

250 angles in S1 head direction cells. Similar results were observed for place cells in the S1

251 without throwing food pellets within the running arena (upper panels in Fig. S20b). 
a

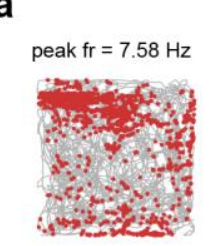

peak $\mathrm{fr}=4.17 \mathrm{~Hz}$
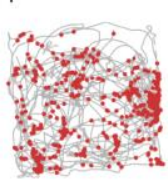

peak $\mathrm{fr}=4.50 \mathrm{~Hz}$
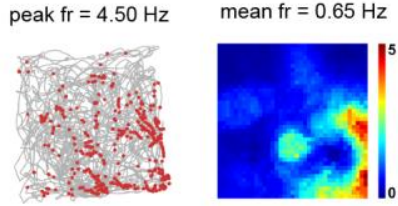

b
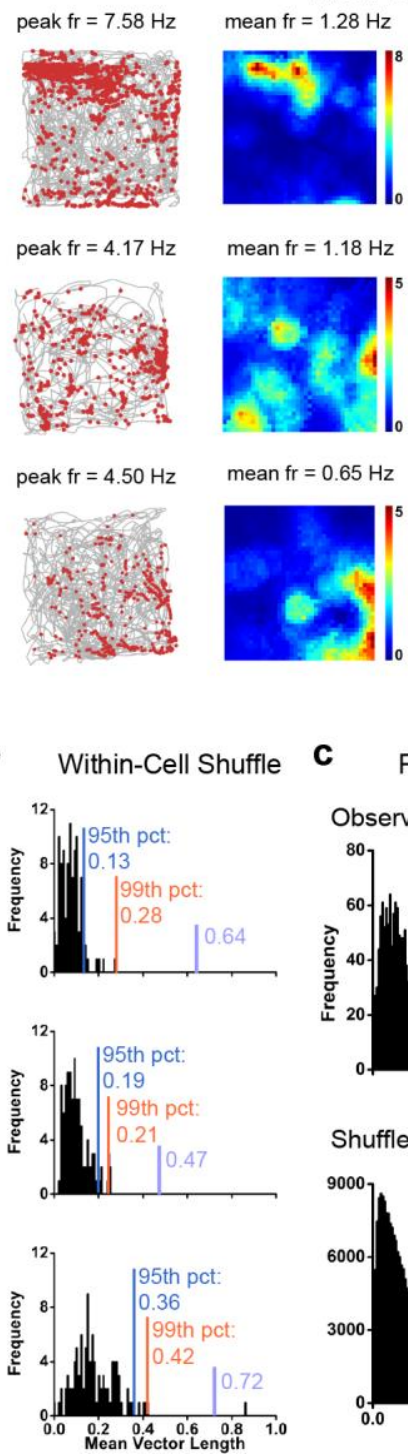

mean $\mathrm{fr}=1.18 \mathrm{~Hz}$

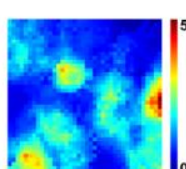

Head Direction Cells
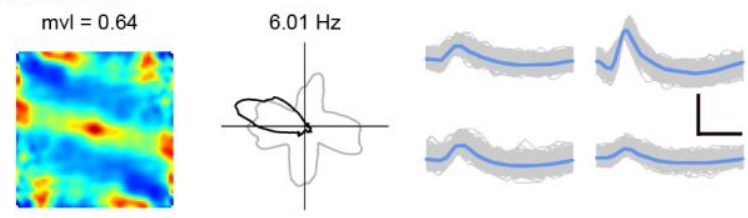

$\mathrm{mvl}=0.47$
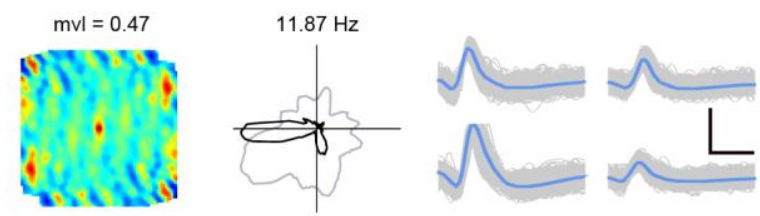

$\mathrm{mvl}=0.72$
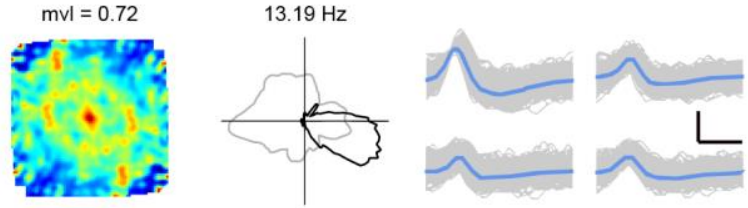

d

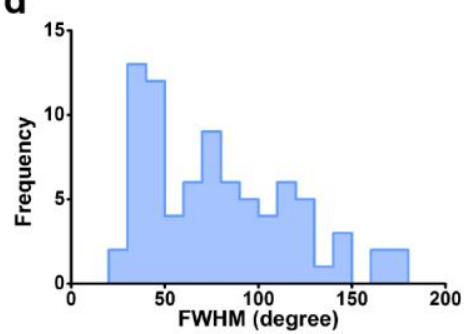

e
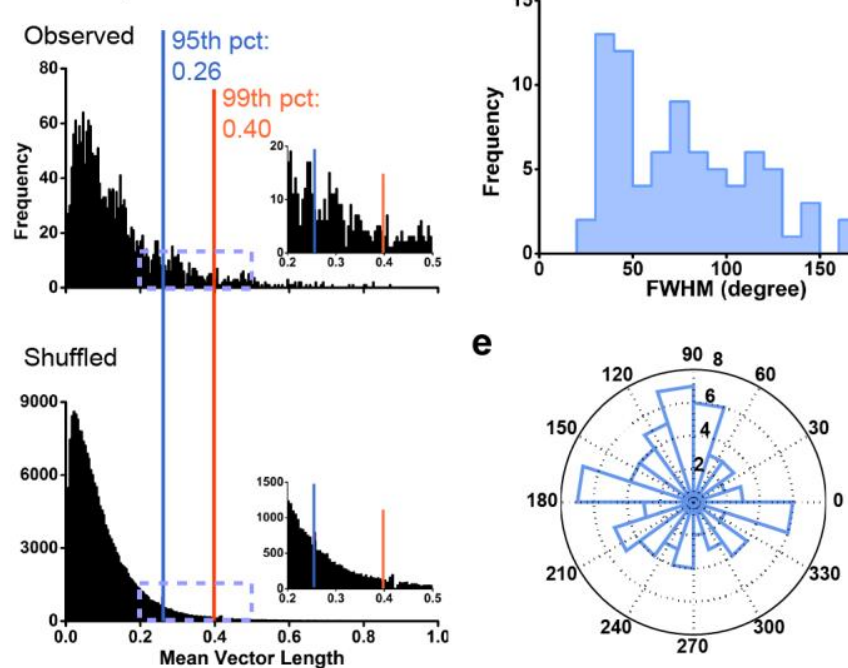

Fig. 2 Head direction cells recorded from the somatosensory cortex.

254 (a) Three examples of somatosensory head direction cells. Trajectory (grey line) with superimposed spike locations (red dots) (left column); spatial firing rate maps (middle left column), autocorrelation diagrams (middle right column) and head direction tuning curves (black) plotted against dwell-time polar plot (grey) (right column). Firing rate is color-coded with dark blue indicating minimal firing rate and dark red indicating maximal firing rate. The scale of the autocorrelation maps is twice that of the spatial firing rate maps. Peak firing rate (fr), mean firing rate (fr), mean vector length (mvl) and angular peak rate for each representative head direction cell are labelled at the top of the panels. The directional plots show strong head direction tuning. Spike waveforms on four electrodes are shown on the right column. Scale bar, $150 \mu \mathrm{V}, 300 \mu \mathrm{s}$. (b) 
Distribution of within-cell shuffled mean vector length for three representative somatosensory head direction cells. The orange and blue stippled lines mark the $99^{\text {th }}$ and the $95^{\text {th }}$ percentile significance level of each randomly shuffled distribution, respectively. The purple line indicates the observed mean vector length. (c) Distribution of mean vector length for all identified somatosensory units. The top panel shows the distribution for observed values. The bottom panel shows the distribution for randomly shuffled data from the same sample. The orange and blue stippled lines mark the $99^{\text {th }}$ and the $95^{\text {th }}$ percentile significance level of each randomly shuffled distribution, respectively. A zoomed panel shows the magnification of the specified area marked by the purple dashed rectangle.

(d) Distribution of head directional tuning width. (e) Preferred direction of recorded head direction cells from S1. The polar plot shows the distribution of the peak firing direction of all identified somatosensory head direction cells. The preferred head direction exhibits a uniform distribution $(P=0.17$, Rayleigh's test).

\section{Border/Boundary vector coding in the somatosensory cortex}

Boundary vector cells and border cells discharge exclusively whenever an animal is physically close, at a specific distance and direction, to one or several environmental boundaries, for example, the enclosure walls of the recording arena ${ }^{4,5,54}$. S1 cells were defined as border cells if border scores (see Methods) were larger than the $99^{\text {th }}$ percentile of population shuffled scores (Fig. 3c). A total of 86/2025 (4.25\%) S1 cells were classified as border cells, and this percentage was significantly higher than expected by random selection from the entire shuffled population (Fig. $3 \mathbf{c} ; Z=14.68$, $P<0.001$; binomial test with expected $P_{0}$ of 0.01 among large samples). Similar results were observed by performing cell-specific shuffling for those three representative somatosensory border cells (Fig. 3b). Most border cells $(n=58,67.44 \%)$ fired along a single boundary while others were active along with two $(n=15,17.44 \%)$, three $(n=$ 6, 6.98\%) or even four $(n=7,8.14 \%)$ boundaries within the enclosure (Fig. 3a and Fig. 
293 spike width were summarized in Fig. S8g. The number of S1 border cells seemed to be 294 higher in layer V than in layer IV or layer VI (Fig. S3b and Fig. S3e). At a lower $95^{\text {th }}$ 295 percentile criterion, 234/2025 (11.56\%) somatosensory cells were classified as border 296 cells.

297 Qualitatively, S1 border cells maintained their border-specific firing fields between 298 circular and square enclosures (Figs. S23a-c), comparable to boundary vector cells and 299 border cells from the presubiculum, parasubiculum and the medial entorhinal cortex 300 (MEC) ${ }^{4,5,17,54}$. S1 border cells also responded to a new wall insert to a familiar 301 enclosure (Figs. S24a-c), as described in the MEC ${ }^{5,54}$. As observed in the MEC and 302 the subiculum ${ }^{4,5}$, removal of walls from the enclosure on an elevated platform 303 preserved similar "border" specificity to that in the walled arena ${ }^{54}$. These results 304 confirmed that somatosensory border cells continued to code for geometric boundaries 305 instead of physical borders in the unwalled environments (Figs. S25a-c). Interestingly, 306 we also detected a certain number of boundary vector cells ${ }^{4,54}$ which discharged further away from the edge of the enclosure (Fig. S26). Further quantitative characterization 308 of somatosensory boundary vector cells combined with the previously described 309 "perimeter" coding might reveal how somatosensory boundary vector cell closely resemble their counterparts in the subiculum ${ }^{1,4,54}$. 
a

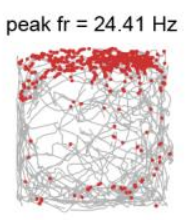

peak $\mathrm{fr}=15.68 \mathrm{~Hz}$
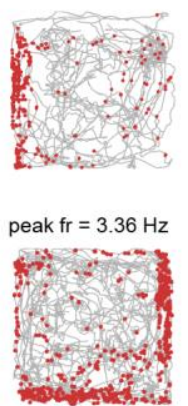

b

Within-Cell Shuffle
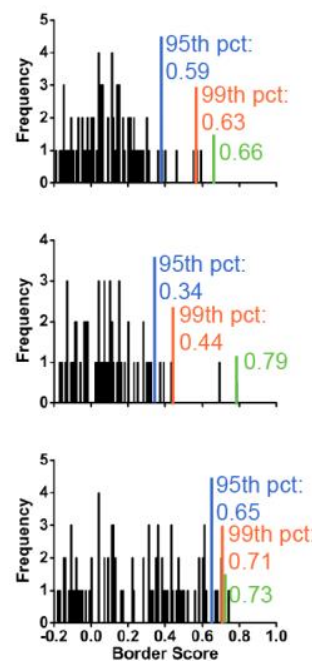

Border Cells
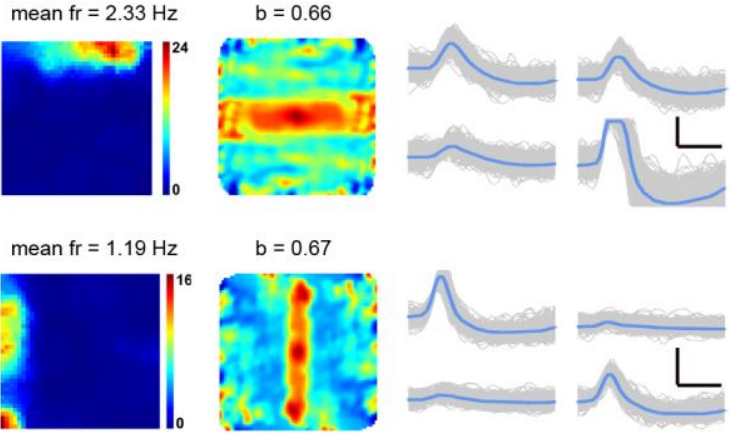

$b=0.67$
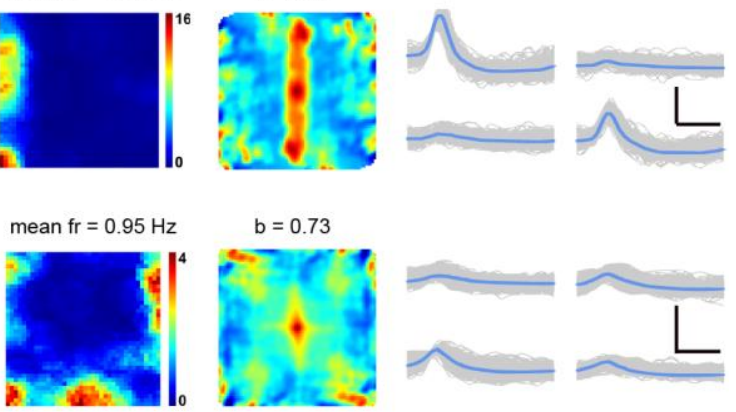

C

Population Shuffle

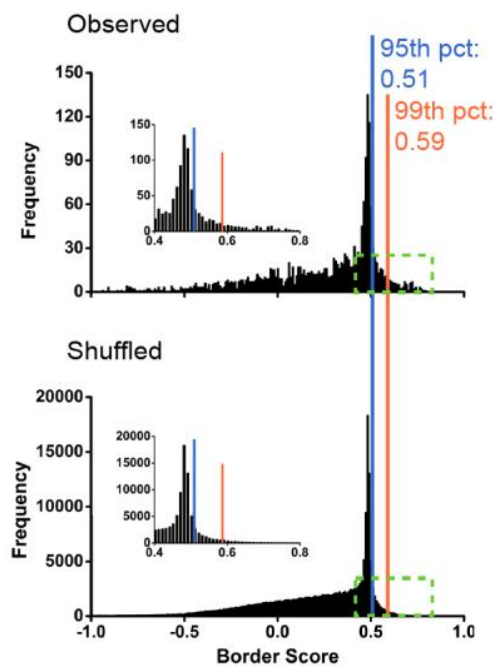

Fig. 3 Border cells recorded from the somatosensory cortex.

313 (a) Three representative examples of somatosensory border cells. Trajectory (grey line)

314 with superimposed spike locations (red dots) (left column); heat maps of spatial firing rate (middle column) and autocorrelation diagrams (right column). Firing rate is colorcoded with dark blue indicating minimal firing rate and dark red indicating maximal firing rate. The scale of the autocorrelation maps is twice that of the spatial firing rate maps. Peak firing rate (fr), mean firing rate (fr) and border score (b) for each representative border cell are labelled at the top of the panels. Spike waveforms on four electrodes are shown on the right column. Scale bar, $150 \mu \mathrm{V}, 300 \mu$ s. (b) Distribution of within-cell shuffled border score for three representative somatosensory border cells. 
322 The orange and blue stippled lines mark the $99^{\text {th }}$ and the $95^{\text {th }}$ percentile significance

323 level of each randomly shuffled distribution, respectively. The green line indicates the

324 observed border score. (c) Distribution of border scores for pooled somatosensory cells.

325 The top panel shows the distribution for observed values. The bottom panel shows the

326 distribution for randomly shuffled versions from the same sample. The orange and blue

327 stippled lines mark the $99^{\text {th }}$ and the $95^{\text {th }}$ percentile significance level of each randomly

328 shuffled distribution, respectively. A zoomed panel shows the magnification of the

329 specified area marked by the green dashed rectangle.

\section{Grid cells in the somatosensory cortex}

Hexagonal firing patterns identified in the MEC ${ }^{6}$ can also be observed in the somatosensory cortex. To quantify the regularity of hexagonal firing patterns of the somatosensory cells (Fig. 4a and Fig. S28), we calculated grid scores based on rotated autocorrelograms of rate maps. A cell was categorized as a grid cell if its grid score was higher than the $99^{\text {th }}$ percentile of the shuffled data. $72 / 2025$ cells $(3.55 \%)$ met this criterion (Fig. 4d), and this percentage was significantly higher than expected by random selection from the entire shuffled population (Fig. 4d; $Z=12.90, P<0.001$; binomial test with expected $P_{0}$ of 0.01 among large samples). Similar results were observed by performing within-cell shuffling (Fig. 4b). To eliminate the possible influence of inhomogeneous sampling on the multiple firing fields of somatosensory grid cells and verify S1 grid cells through spike shuffling, we performed additional "field shuffling" 22 and found that these exampled grid cells also passed the gridness threshold for the "field shuffling' procedure (Fig. 4c). The average firing rate and the grid score were $1.18 \pm 0.12 \mathrm{~Hz}$ (Fig. S8d) and $0.55 \pm 0.02$ (Fig. S4d), respectively. The histograms of peak-to-peak amplitude and peak-to-trough spike width were presented in Fig. S8h.

Grid spacing in somatosensory grid cells (Fig. 4e) was $42.72 \pm 1.30 \mathrm{~cm}$, and the average grid size was $842.44 \pm 54.22 \mathrm{~cm}^{2}$ (Fig. 4f). The fluctuation in somatosensory grid spacings is not a result of interindividual differences across different animals (Fig. S4d). 
Moreover, the orientation of the somatosensory grid cells varied and was on the average found to be $32.63 \pm 2.64$ degrees (Fig. 4g). Again, with a lower threshold at the $95^{\text {th }}$ percentile, 218/2025 S1 neurons (10.37\%) were classified as grid cells, with the grid score threshold of 0.17 (blue line in Fig. 4d). When recorded in a larger environment (1.5 $\mathrm{m} \times 1.5 \mathrm{~m}$ box), the discharging patterns of somatosensory grid cells showed similar multiple regular triangular structures in a larger enclosure to those within the smaller enclosure (1.0 m x $1.0 \mathrm{~m}$ ) with a high grid score (Figs. S29a-c), confirming that those identified grid cells were not false-positive grid cells due to accidental triangular node structures. It appeared that somatosensory grid cells were less prevalent and a little noisier than place cells, head direction cells and grid cells identified in the S1. It remains to be determined whether multisensory inputs and intrinsic oscillatory dynamics within the somatosensory cortex might generate different rate-coded grid computations from those within the hippocampal-entorhinal cortex. Taken together, the local configuration of grid cells in the somatosensory cortex exhibited similar features as those in MEC. Furthermore, we detected irregular grid cells with negative grid scores in the S1 along with regular grid cells (Fig. 4 h and Fig. S31).

a
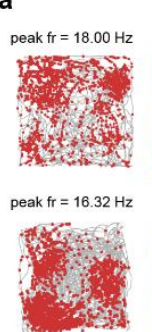

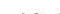

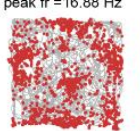

d

d Population Shuffle
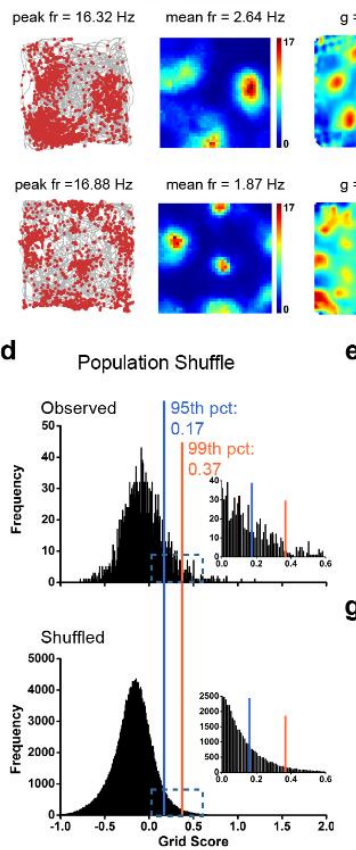

Grid Cells
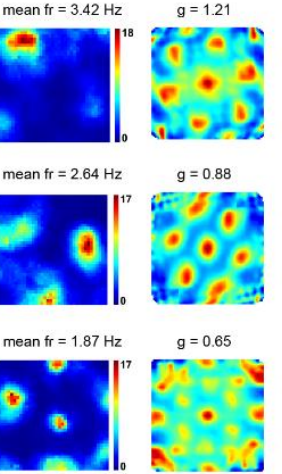

e

हुำ
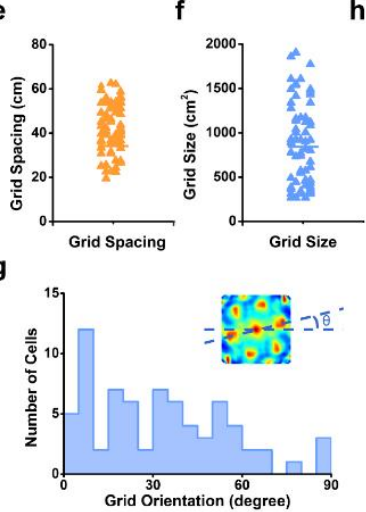
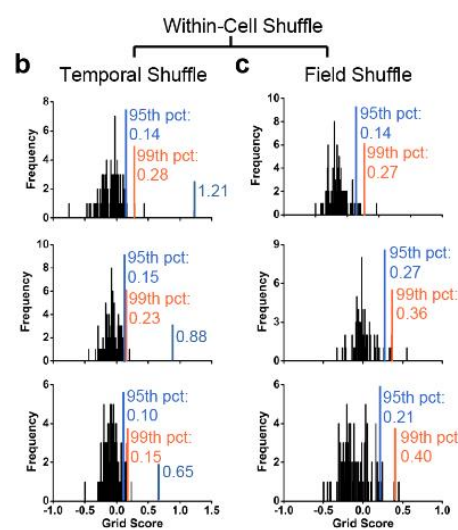

h
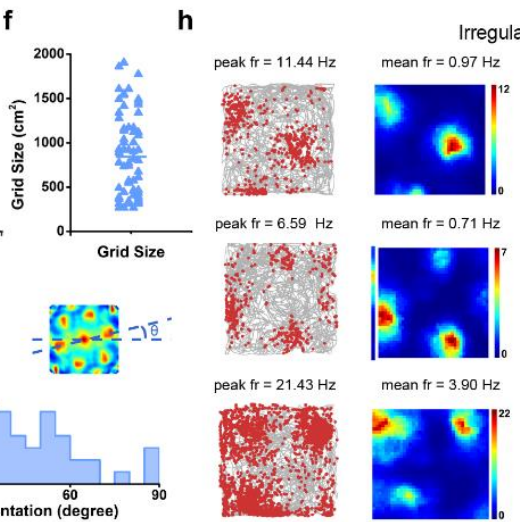

regular Grid Cells
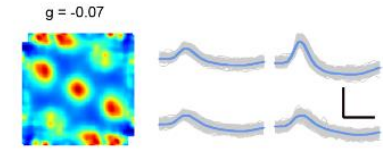

$g=-0.11$
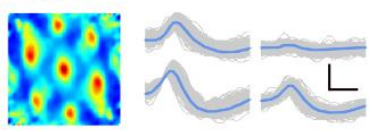

$g=0.20$
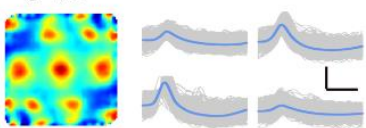


\section{Fig. 4 Grid cells in the somatosensory cortex.}

(a) Three representative examples of somatosensory grid cells. Trajectory (grey line) with superimposed spike locations (red dots) (left column); heat maps of spatial firing rate (middle column) and autocorrelation diagrams (right column). Firing rate is colorcoded with dark blue indicating minimal firing rate and dark red indicating maximal firing rate. The scale of the autocorrelation maps is twice that of the spatial firing rate maps. Peak firing rate (fr), mean firing rate (fr) and grid score ( $g$ ) for each representative grid cell are labelled at the top of the panels. A crystal-like hexagonal firing pattern was observed. Spike waveforms on four electrodes are shown on the right column. Scale bar, $100 \mu \mathrm{V}, 300 \mu \mathrm{s}$. (b) Distribution of within-cell shuffled grid score for three representative somatosensory grid cells. The orange and blue stippled lines mark the $99^{\text {th }}$ and the $95^{\text {th }}$ percentile significance level of each randomly shuffled distribution, respectively. The cyan line indicates the observed grid score. (c) The same as (b) but for field shuffle. (d) Distribution of grid scores for somatosensory cells. The top panel shows the distribution for observed values. The bottom panel shows the distribution for randomly shuffled versions from the same sample. The orange and blue stippled lines mark the $99^{\text {th }}$ and the $95^{\text {th }}$ percentile significance level of each randomly shuffled distribution, respectively. A zoomed panel shows the magnification of the specified area marked by the cyan dashed rectangle. (e-f) The raster plots show the distribution of grid spacing and grid size of the classified somatosensory grid cells. (g) The histogram of grid orientation from categorized somatosensory grid cells. (h) Three representative examples of irregular somatosensory grid cells. Spike waveforms on four electrodes are shown on the right column. Scale bar, $150 \mu \mathrm{V}, 300 \mu \mathrm{s}$.

\section{Conjunctive cells in the somatosensory cortex}

To establish the existence of S1 neurons coding more than one spatial correlate, we assessed the strength of head direction tuning for all somatosensory place cells, border cells and grid cells ${ }^{7}$. A certain number of somatosensory neurons were found to be conjunctive cells (Fig. 5 and Fig. S3a). Specifically, 25/195 place cells (Fig. 5a), 11/86 border cells (Fig. 5b) and 5/72 grid cells (Fig. 5c) were significantly tuned by head 
direction. Interestingly, we also detected irregular hexagonal grid cells modulated with head direction (Fig. 5d).

The degree of directionality in conjunctive cells showed no obvious difference from that of pure head direction cells (Watson's $\mathrm{U}^{2}$ test, $P=0.09$ ). These results suggested that the somatosensory cortex carried similar conjunctive spatial $\mathrm{x}$ directional, border $\mathrm{x}$ directional and gird $\mathrm{x}$ directional signal to those identified previously in the rat subiculum ${ }^{45}$ and the rat MEC ${ }^{5,7}$.

a

Conjunctive Place $\times$ Head Direction Cell
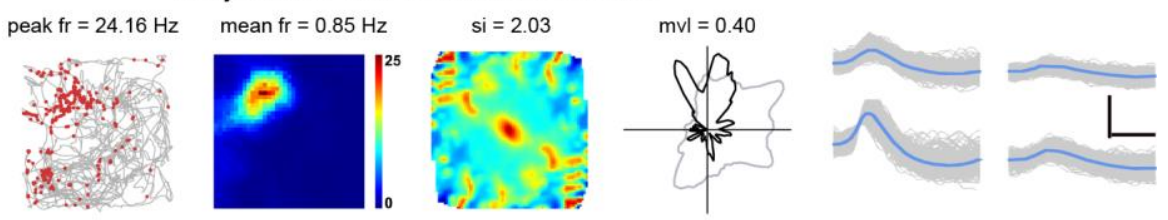

b

Conjunctive Border $\times$ Head Direction Cell
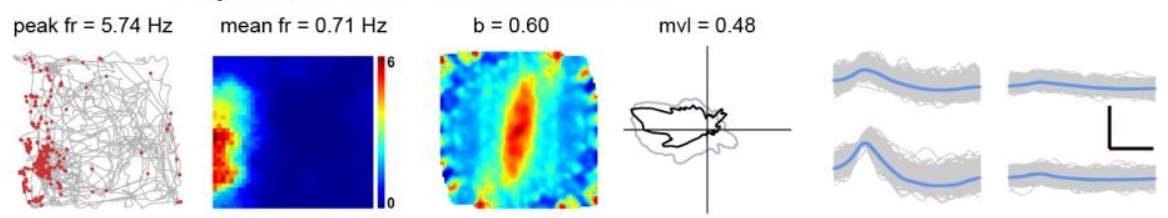

C

Conjunctive Grid $\times$ Head Direction Cell
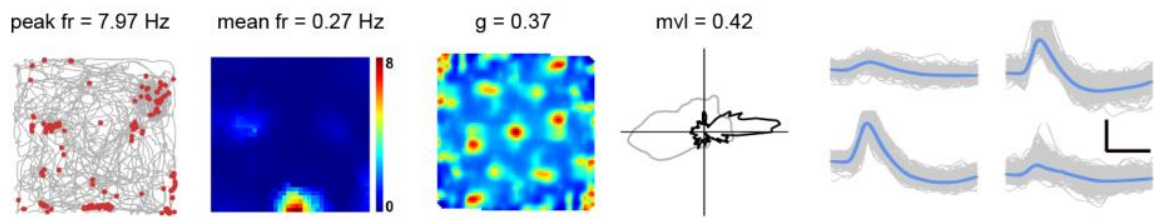

d

Conjunctive Irregular Grid $\times$ Head Direction Cell
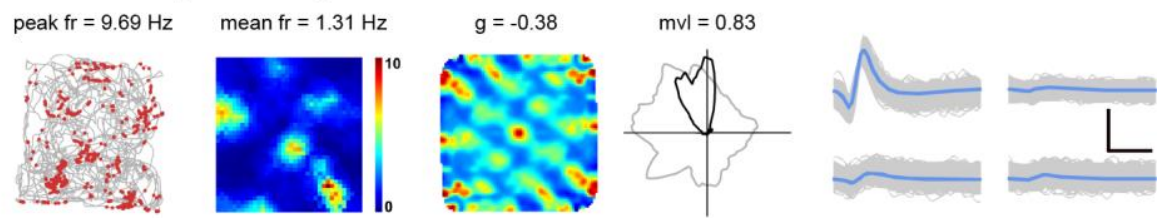

Fig. 5 Four different types of conjunctive cells in the somatosensory cortex.

(a) A representative conjunctive place $\mathrm{x}$ head direction cell. (b) A representative conjunctive border $\mathrm{x}$ head direction cell. (c) A representative conjunctive grid $\mathrm{x}$ head direction cell. (d) A representative conjunctive irregular grid $\mathrm{x}$ head direction cell. Trajectory (grey line) with superimposed spike locations (red dots) (left column); heat maps of spatial firing rate (middle left column), autocorrelation diagrams (middle right column) and head direction tuning curves (black) plotted against dwell-time polar plot 
411 (grey) (right column). Firing rate is color-coded with dark blue indicating minimal

412 firing rate and dark red indicating maximal firing rate. The scale of the autocorrelation 413 maps is twice that of the spatial firing rate maps. Peak firing rate (fr), mean firing rate 414 (fr), spatial information (si), border score (b), grid score (g) and mean vector length 415 (mvl) for each representative cell are labelled at the top of the panels. Spike waveforms on four electrodes are shown on the right column. Scale bar, $150 \mu \mathrm{V}, 300 \mu \mathrm{s}$.

\section{Impact of the whisker trimming on the somatosensory spatial activities}

Since rodents use their whiskers to actively explore the external environment for whisker-based navigation 55, 56, we implanted two additional rats (Fig. S1b) and recorded neuronal activity before and after repeated whisker trimming (Fig. S32a and Fig. S32c) to gauge the involvement of the vibrissae. Two consecutive recording sessions from the same rat before and right after whisker trimming showed that the distribution of clusters and waveforms in the S1 was qualitatively similar (Fig. S32b and Fig. S32d).

To further quantify the change of well-isolated spike clusters before and after whisker trimming, we calculated the isolation distance and L-ratio ${ }^{42}$. The median isolation distances were 122.35 and 132.9 before and after whisker trimming, respectively. There was no significant difference in cluster separation and L-ratio in the S1 before and right after whisker trimming (Fig. S32e and Fig. S32f, $n=78, P=0.73$ and 0.76 , respectively, two-tailed paired $t$-test). The average drift of center of mass for the total cell sample before and after whisker trimming was $0.13 \pm 0.01$, indicating there was minimal change in spike clusters before and after whisker trimming. Besides, both peak and average firing rates of the same neurons before and after whisker trimming did not change significantly (Fig. S32g and Fig. S32h, $n=78, P=0.75$ and 0.47 , respectively, two-tailed paired $t$-test).

To further assess whether whisker trimming affected the S1 spatial firing properties, we were able to continuously record the same S1 border cell before and after whisker 
trimming. The spatial firing patterns of the border cell persisted and responded to a new wall inserted into the square enclosure (Fig. S33a and Fig. S33b). Overall, both the spatial responses and firing properties in the $\mathrm{S} 1$ were not significantly affected by the whisker trimming.

When we pooled and analyzed a total of 461 cells recorded after whisker trimming, we were still able to identify all different spatial cell types including 43 place cells (Fig. 6a), 15 head direction cells (Fig. 6b), 29 border cells (Fig. 6c) and 17 grid cells (Fig. 6d). The percentage of all recorded spatially tuned cell types after whisker trimming were similar to those recorded from rats without whisker trimming. Furthermore, the cutoffs for defining place cells (Fig. 6e), head direction cells (Fig. 6f), border cells (Fig. 6g) and grid cells (Fig. 6h) after whisker trimming highly resembled those for different somatosensory spatial cell types without whisker trimming. Taken together, the persistence of recorded somatosensory spatial cells after whisker trimming demonstrated that our identified cells were not significantly influenced by the whisker 452 trimming.

a

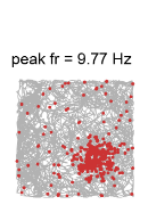

c

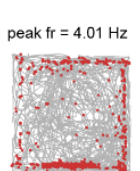

e

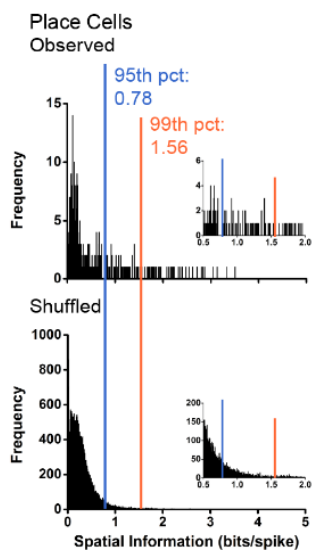

Place Cell

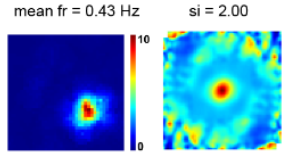

Border Cell
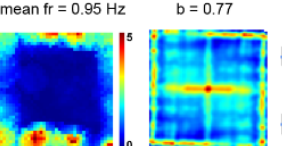
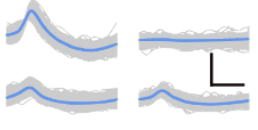

d

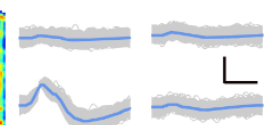

peak fr $=9.81 \mathrm{~Hz}$

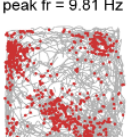

f Head Direction Cells Observed
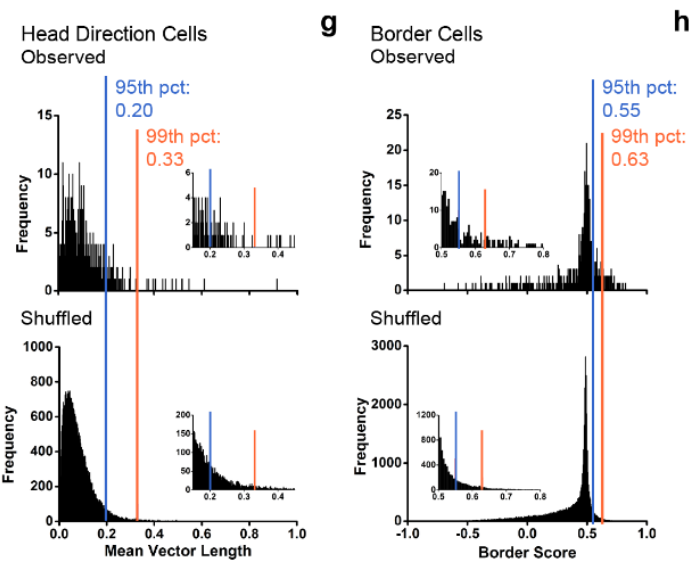

Grid Cells Observed

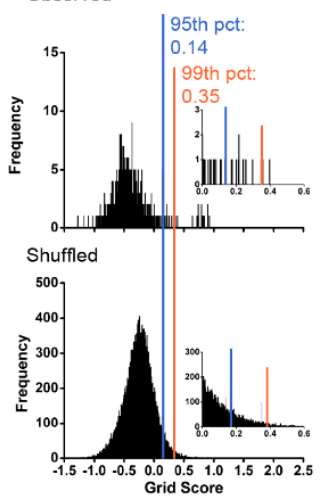


455 (a-d) Four representative examples of somatosensory place cell (a), head direction cell (b), border cell (c) and grid cell (d) after whisker trimming. Trajectory (grey line) with 457 superimposed spike locations (red dots) (left column); spatial firing rate maps (middle left column), autocorrelation diagrams (middle right column) and head direction tuning curves (black) plotted against dwell-time polar plot (grey) (right column). Firing rate is color-coded with dark blue indicating minimal firing rate and dark red indicating maximal firing rate. The scale of the autocorrelation maps is twice that of the spatial

462 firing rate maps. Peak firing rate (fr), mean firing rate (fr), spatial information (si), mean vector length (mvl), border score (b) or grid score (g) and angular peak rate for each representative cell are labelled at the top of the panels. Spike waveforms on four electrodes are shown on the right column. Scale bar, $150 \mu \mathrm{V}, 300 \mu$ s. (e-h) Distribution of spatial information (e), mean vector length (f), border score $(\mathbf{g})$ or grid score $(\mathbf{h})$ for somatosensory cells after whisker trimming. The top panel shows the distribution for observed values. The bottom panel shows the distribution for randomly shuffled data from the same sample. The orange and blue stippled lines mark the $99^{\text {th }}$ and the $95^{\text {th }}$ percentile significance level of each randomly shuffled distribution, respectively.

\section{Discussion}

472 This study provides the first demonstration and characterization of spatially tuned neuronal discharges in the primary somatosensory cortex. We have shown place, grid,

474 boundary vector/border, head direction and conjunctive cell activities, as reported in parahippocampal and associated areas ${ }^{39}$, to co-exist in the single-domain primary somatosensory cortex. These spatially tuned activities encoded within the newly

477 identified somatosensory spatial navigation system appear to be specific, stable, 478 anchored to external visual cues and robust against non-spatial environmental 479 perturbations.

480 Based on the assumption that proprioception makes a significant contribution to spatial representation in the parahippocampal cortices ${ }^{39,40}$, we predicted spatially selective activities should exist in the somatosensory cortex. To our surprise, we were able to 
record the full complement of spatially selective cells (place, grid, boundary vector/border, head direction and conjunctive cells) in the primary somatosensory cortex. Given the paucity of direct projections from the parahippocampal areas to the S1 region ${ }^{57,58}$, we assume somatosensory spatial activities do not arise from the hippocampal-entorhinal circuit. However, we cannot completely eliminate the possibility that the somatosensory spatial positioning system may be at least partially dependent on the hippocampal-entorhinal system. Whether the newly found somatosensory spatial navigation system is independent of, parallel with or convergent onto the classical one within the hippocampal-entorhinal microcircuit remains an interesting question and needs to be addressed in future studies ${ }^{1}$. The availability of single-cell transcriptomics and connectomics for the somatosensory cortex might help to unravel the interaction or interdependence between these two genetically preconfigured spatial maps 59,60 . Since there is very little overlap between septal projections to the hippocampal-entorhinal areas ${ }^{61}$, and that septal inactivation severely attenuates place and grid cell activities in both regions ${ }^{62-64}$, how $\mathrm{S} 1$ responds to septal inactivation may provide the necessary insight for hippocampal-entorhinal dependency.

The laminar organization of the S1 in relation to connectivity and function has been well-characterized, largely arising from studies on the vibrissae barrel fields ${ }^{65,66}$. There is insufficient information on the specific connectivity patterns of S1 in the rat, but mouse data suggest similar cortico-cortical, thalamic-cortical, cortical-thalamic, cortico-striatal and corticofugal connection patterns between S1 and other parts of the $\mathrm{S} 1{ }^{67}$. Our current study is roughly limited to sampling layers IV through VI. There is a pattern for head direction cells to be absent in layer VI and place cells to predominate in deeper layers (Figs. S3e-h). From such distribution of spatial cell types across cortical depth, we could infer that head direction information is not present in corticothalamic communications, given the lack of head direction cells at depths corresponding to layer VI in the somatosensory cortex. There also appears to be a gap in the most superficial aspect of layer V (Va) where all spatial cell types appear to be under-represented. Interestingly, layer Va projections are mostly recurrent ${ }^{68}$ and appear 
to serve as a trans-columnar and trans-laminar integration hub within the $\mathrm{S} 1{ }^{69-71}$. Layer

Va may serve as an area that integrates spatial and proprioceptive information in the $\mathrm{S} 1$, hence salient spatial activity is not readily observable in this layer. If true, this would also suggest that the major extra-cortical output from S1 combines, but do not contain conventional spatial information as characterized here. A full sampling of cortical layers in S1 using high-density linear probes ${ }^{72}$ is necessary to address how spatial information is generated, integrated and distributed elsewhere (e.g. thalamic, striatal and brainstem targets) in the brain.

As mentioned above, there is a lack of direct connections between $\mathrm{S} 1$ and areas that are traditionally associated with spatial processing. Where do the spatial signals come from? Are they more universal in cortical processing than previously thought? What is the functional significance of spatial activities in the S1, or S1 in general? While extensive intra and inter columnar/laminar exist in the S1 and may support de novo generation of place cells (from integrating local grid cell activities) and conjunctive cells, it is unlikely the full complement of known spatial representations are generated independently within the S1. We have demonstrated that S1 place fields and preferred head direction rotate with the varied positioning of salient visual cue as cells found in the hippocampal-entorhinal circuit. This observation suggests the spatial activities cannot solely be driven by proprioception arising from lemniscal/paralemniscal pathways. While a sparse, direct and functional connection exists between the S1 and the visual cortex ${ }^{73}$, it is unlikely to account for visually anchored spatial tuning in the somatosensory cortex. Instead, S1 spatial activities are more likely to be an efference copy from elsewhere in the brain.

S1 functions and activities are intricately linked to motor structures, especially the motor cortex. Functional coupling between S1 spindle activity and spontaneous muscle twitches can be observed as early as two days after birth ${ }^{74}$. It is known that both primary 538 and secondary motor cortex are reciprocally connected with S1 75, 76, and the preparation, execution and dynamic changes in movements can all be predicted from 
$540 \mathrm{M} 1 / \mathrm{S} 1$ activities $^{77}$. In fact, $\mathrm{S} 1$ has recently been shown to have an active role in directly 541 controlling motor output ${ }^{78}$. Position-dependent neuronal discharges (i.e. place field542 like representations) relating to hand movement through space are well-established in 543 the primate motor cortex ${ }^{79}$. In fact, considering movement of the hand or forelimb in general: speed ${ }^{79,80}$, (hand) direction ${ }^{79}$ and conjunctive ${ }^{79,80}$ coding in the motor cortices can be seen as homologous to their spatial navigation counterparts in the limbic system. Essentially, it appears the motor cortex contains spatially tuned activities for specific body parts that are analogous to limbic activities that appear to represent whole body/head movement. Therefore, whole body/head and limb movements through space may require similar neurocomputation, of which is also present in the S1 in addition to motor and limbic cortices. This proposal is also consistent with the idea that S1

551 simulates the body itself in addition to its representation, which requires spatial

\section{2}

553 information ${ }^{37}$. From this synthesis, we speculate that we would be able to record spatially tuned activities in the limb region of the motor cortex, as well as other sensorimotor cortices (e.g. the forelimb and trunk regions). Particularly, inactivation of the reciprocally connected motor cortex (i.e. limb region of the motor cortex) can test if S1 spatial activities are efference copy from the motor cortex. Recent reports of place cell-like activities in the primate sensorimotor cortices ${ }^{38}$ during quasi-active movements through space support the notion that spatial representation of body movement may exist throughout the cortex.

Our discussion so far favors somatosensory spatial activity may be an efferent copy from the motor cortex. If true, then the question remains as to how an area such as the motor cortex without direct connectivity with spatially tuned limbic structures (like the S1) can acquire spatial activities. To the best of our knowledge, no grid- or boundary vector-/border-like spatial representation has been reported in the motor cortex in conventional reaching studies, although this discrepancy may be related to that motor tasks in such studies are often limited to a single defined trajectory (insufficient spatial coverage to detect grid cells) in open task space (without physical borders). Likewise, the "body simulation" model ${ }^{37}$ predicts the existence of body part location information 
569 (which we interpret to be place cell-like representations) to exist in layer IV, but our 570 data indicate the full complement of spatial activities can be detected from at least

571 layers IV through VI. Our data is not fully compatible with currently available 572 experimental and theoretical work in explaining why all currently recognized spatial 573 cell activities can be found in S1 and how they are generated. Regardless, we propose 574 it is likely spatial information is integral to the "body simulation" function in S1 as put 575 forward by Brecht ${ }^{37}$. High-resolution kinematic studies ${ }^{81}$ of body part (e.g. limb) 576 movements through space would be required to test if spatial activities in the S1 do in 577 fact represent body parts in space.

578 In this report, we have demonstrated place, grid, boundary vector/border, head direction 579 and conjunctive cell activities in the primary somatosensory cortex. These activities are comparable to their counterparts in the parahippocampal cortices and are stable, 581 specific, robust to non-spatial manipulations and anchored to salient spatial cues. We

582 propose spatial tuning in the primary somatosensory cortex may be crucial for body representation/simulation in space, and the newly identified somatosensory spatial representation system may generalize to other sensorimotor cortices pertinent to behavioral coordination.

\section{Materials and Methods}

\section{Subjects}

Ten male Long-Evans rats (2-4 months old, 250-450 grams at the time of the surgery) were used for this study. All animals were singly housed in transparent cages $(35 \mathrm{~cm} \mathrm{x}$ $45 \mathrm{~cm} \times 45 \mathrm{~cm}, \mathrm{~W} \times \mathrm{L} \times \mathrm{H}$ ) and maintained on a 12-hour reversed light-dark cycle (lights on at 9 p.m. and off at 9 a.m.). Experiments were performed during the dark phase. Rats were maintained in a vivarium with controlled temperature $\left(19-22^{\circ} \mathrm{C}\right)$, humidity (55-65\%). and were kept at about 85-90\% of free-feeding body weight. Food restriction was imposed 8-24 hours before each training and recording trial. Water was available ad libitum. All animal experiments were performed in accordance with the 
596 National Animal Welfare Act under a protocol approved by the Animal Care and Use

597 Committee from both Army Medical University and Xinqiao Hospital.

\section{Surgery and tetrode placement}

599 Rats were anesthetized with isoflurane. Microdrives loaded with four tetrodes were 600 implanted to target the hindlimb (S1HL), forelimb (S1FL) and shoulder (S1Sh) regions 601 of the primary somatosensory cortex (anterior-posterior (AP): 0.2-2.2 $\mathrm{mm}$ posterior to 602 bregma; medial-lateral (ML): 2.2-3.4 mm lateral to midline, dorsal-ventral (DV): 603 0.4/0.6-3 mm below the dura.), secured with dental cement with 8-10 anchor screws. A 604 screw served as the ground electrode. Tetrodes were assembled with four $17 \mu \mathrm{m}$ 605 Platinum/Iridium wires (\#100167, California Fine Wire Company). Tetrodes had 606 impedances between 150 and $300 \mathrm{k} \Omega$ at $1 \mathrm{kHz}$ through electroplating (nanoZ; White

607

608

609 Matter LLC). 8-10 jeweler screws were attached into the rat skull, and individual microdrives were anchored to screws with several rounds of application of the dental cement.

\section{Training and data collection}

Behavioral training, tetrode turning and data recording started one-week post-surgery. Rats were trained to run around in a $1 \mathrm{~m}$ x $1 \mathrm{~m}$ square box with a white cue card (297 $\mathrm{mm} \times 210 \mathrm{~mm}$ ) mounted on one side of the wall. Food pellets were scattered into the arena intermittently to encourage exploration.

Each recording session lasted between 10 and $30 \mathrm{~min}$ to facilitate full coverage of the testing arena. Tetrodes were lowered in steps of 25 or $50 \mu \mathrm{m}$ daily until well-separated single units can be identified. Data were acquired by an Axona system (Axona Ltd., St. Albans, U.K.) at $48 \mathrm{kHz}$, band-passed between .8-6.7 kHz and a gain of x5-18k. Spikes were digitized with 50 8-bit sample windows. Local field potentials were recorded from one of the electrodes with a low-pass filter $(500 \mathrm{~Hz})$.

\section{Spike sorting, cell classification and rate map}

Spike sorting was manually performed offline with TINT (Axona Ltd, St. Albans, 
623 U.K.), and the clustering was primarily based on features of the spike waveform (peak624 to-trough amplitude and spike width), together with additional autocorrelations and 625 cross-correlations ${ }^{44,82}$. During our manual cluster cutting, we always counted neurons

626

627

628

629

630

631

632

633 with similar or identical waveform shapes only once whenever similar or identical individual neurons were recorded and tracked across two consecutive recording sessions. To confirm the quality of cluster separation, we calculated L-ratio as well as isolation distance between clusters.

Two small light-emitting diodes (LEDs) were mounted to the headstage to track the rats' speed, position and orientation via an overhead video camera. Only spikes with instantaneous running speeds $>2.5 \mathrm{~cm} / \mathrm{s}$ were chosen for further analysis in order to exclude confounding behaviors such as immobility, grooming and rearing.

To classify firing fields and firing rate distributions, the position data were divided into $2.5-\mathrm{cm} \times 2.5-\mathrm{cm}$ bins, and the path was smoothed with a 21 -sample boxcar window filter (400 ms; 10 samples on each side $)^{7,17,43}$. Cells with > 100 spikes per session and with a coverage of $>80 \%$ were included for further analyses. Maps for spike numbers and spike times were smoothed with a quasi-Gaussian kernel over the neighboring $5 \mathrm{x}$ 5 bins $^{7,17,43}$. Spatial firing rates were calculated by dividing the smoothed map of spike numbers with spike times. The peak firing rate was defined as the highest rate in the corresponding bin in the spatial firing rate map. Mean firing rates were calculated from the whole session data.

\section{Analysis of place cells}

Spatial information is a quantification of the extent to which a neuron's firing pattern can predict the position of freely moving animals and is expressed in the unit of bits per spike. The spatial information was calculated as:

$$
\text { spatial information }=\sum_{i} p_{i} \frac{\lambda_{i}}{\lambda} \log _{2} \frac{\lambda_{i}}{\lambda}
$$


where $\lambda_{i}$ is the mean firing rate of the cell in the $i$-th bin, $\lambda$ is the overall mean firing rate of the cell in the trial, and $p_{i}$ is the probability for the animal being at the location of the $i$-th bin.

Adaptive smoothing ${ }^{44}$ was applied to optimize the trade-off between spatial resolution and sampling error before the calculation of spatial information. The data were first divided into $2.5-\mathrm{cm} \times 2.5-\mathrm{cm}$ bins, and then the firing rate within each bin was calculated by expanding a circle centered on the bin until

$$
\gamma \geq \frac{\alpha}{n \sqrt{s}}
$$

where $\gamma$ is the circle's radius in bins, $n$ is the number of occupancy of samples within the circle, $s$ is the total number of spikes fired within the circle, and $\alpha$ is a constant parameter set to 10,000 . With a position sampling frequency at $50 \mathrm{~Hz}$, the firing rate assigned to that bin was then set to $50 \cdot s / n$.

A place cell was classified as a cell with the spatial information above chance level, which was computed by a random permutation process using all recorded cells. For each round of the shuffling process, the entire sequence of spike trains from each cell was time-shifted along the animal's trajectory by a random period between $20 \mathrm{~s}$ and the trial duration minus $20 \mathrm{~s}$, with the end wrapped to the beginning of the trial. A spatial firing rate map was then constructed, and spatial information was calculated. This shuffling process was repeated 100 times for each cell, generating a total of 202,500 permutations for the 2025 somatosensory neurons. This shuffling procedure preserved the temporal firing characteristics in the unshuffled data while disrupting the spatial structure at the same time.

Spatial information score was then measured for each shuffled rate map. The distribution of spatial information values across all 100 permutations of all cells was computed and finally, the $99^{\text {th }}$ percentile of the significant level was determined. The threshold values for categorizing cells into place cells were defined as the spatial 
674 information scores above the $99^{\text {th }}$ percentile of the distribution from shuffled

675 populations. In addition to the population shuffling, the within-cell shuffling was also performed within the entire sequence of spike trains from each individual neuron, and the shuffling process was also repeated 100 times for each single cell.

678

Spatial sparsity was used to measure how compact and selective the place field of each place cell is relative to the recording enclosure. The spatial sparsity was calculated using the formula as follows ${ }^{44}$ :

$$
\text { Sparsity }=\frac{\left(\sum p_{i} \lambda_{i}\right)^{2}}{\sum p_{i} \lambda_{i}^{2}}
$$

Where $p_{i}$ is the occupancy probability for the animal being at the location of the $i$-th bin in the map and $\lambda_{i}$ is the mean firing rate of the cell in bin $i$.

Spatial coherence was estimated by calculating the mean correlation between the firing rate of each bin in the map and the aggregate firing rate of the eight nearest bins ${ }^{34}$. We used unsmoothed firing rate maps for computing the spatial coherence.

The spatial correlation across trials from the same recording arena was computed for each cell by correlating the firing rates in corresponding paired bins of two smoothed rate maps. The spatial stability within trials was estimated by calculating spatial (2D) correlations between firing rate maps generated from the first and second halves of the same trial. Place cells with spatial stability lower than 0.3 were excluded for further analysis.

\section{Analysis of grid cells}

Spatial autocorrelation was calculated with smoothed rate maps 7, 17, 43 . Autocorrelograms were derived from Pearson's product-moment correlation coefficient correcting for edge effects and unvisited locations. 
697 With $\lambda(x, y)$ representing the average firing rate of a cell at coordinate $(x, y)$, the 698 autocorrelation between the spatial firing field itself and the spatial firing field with lags 699 of $\tau_{x}$ and $\tau_{y}$ was calculated as:

$$
\mathrm{r}\left(\tau_{x}, \tau_{y}\right)
$$

$$
=\frac{\mathrm{n} \sum \lambda(x, y) \lambda\left(x-\tau_{x}, y-\tau_{y}\right)-\sum \lambda(x, y) \sum \lambda\left(x-\tau_{x}, y-\tau_{y}\right)}{\sqrt{n \sum \lambda(x, y)^{2}-\left[\sum \lambda(x, y)\right]^{2}} \sqrt{n \sum \lambda\left(x-\tau_{x}, y-\tau_{y}\right)^{2}-\left[\sum \lambda\left(x-\tau_{x}, y-\tau_{y}\right)\right]^{2}}}
$$

where the summation is over whole $\mathrm{n}$ pixels in $\lambda(x, y)$ for which firing rate was calculated for both $\lambda(x, y)$ and $\lambda\left(x-\tau_{x}, y-\tau_{y}\right)$. Autocorrelations were not calculated for spatial lags of $\tau_{x}, \tau_{y}$ where $n<20$.

The degree of spatial regularity ("gridness" or "grid score") was calculated for each unit by using a circular sample centered on the central peak of the autocorrelogram but excluding the central peak itself, and by comparing rotated versions of this circular sample ${ }^{5,7}$. The Pearson's correlations between this circular sample and its rotated versions were calculated, with the angles of rotation of $60^{\circ}$ and $120^{\circ}$ in the first group, and $30^{\circ}, 90^{\circ}$ and $150^{\circ}$ in the second group. Gridness or the neuron's grid score was defined as the minimal difference between any of the coefficients in the first group and any of the coefficients in the second group. Shuffling was performed in the same procedure used for defining place cells. Grid cells were categorized as cells with the rotational symmetry-based grid scores exceeding the $99^{\text {th }}$ percentile of the distribution of grid scores for shuffled data from the entire population of identified somatosensory cells.

Grid spacing was computed as the median distance from the grid center to the closest peak among six neighboring firing fields in the autocorrelogram of the spatial firing map. Since such analysis is sensitive to noise in the grid autocorrelogram, grid spacing was computed only when the median distance to the six neighboring peaks for the analyzed cell was comparable to the radius of the circle centered on the gird 
autocorrelogram with the highest grid score. The radius of this circle around the center of the autocorrelogram was also referred to as the grid field size.

Grid orientation was calculated by first computing vectors from the center of the autocorrelogram to each of the three adjacent peaks among six neighboring firing fields in the autocorrelogram of the spatial firing map in the counterclockwise direction, beginning with a camera-based reference line of zero degree. The angle between the minimal orientation of those three vectors and the camera-based reference line was defined as the grid orientation.

\section{Analysis of head direction cells}

The rat's head direction was estimated by the relative position of the LEDs differentiated through their sizes ${ }^{7,17,43}$. The directional tuning curve for each recorded cell was drawn by plotting the firing rate as a function of the rat's head angle, which is divided into bins of 3 degrees and then smoothed with a 14.5-degree mean window filter ( 2 bins on each side). To avoid bias, data were only used if all head angle bins contain data.

The strength of directionality was calculated by computing the mean vector length from circular distributed firing rates. The chance values were determined by a shuffling process simulated in the same way as for place cells, with the entire sequence of spike trains time-shifted between $20 \mathrm{~s}$ and the whole trail length minus $20 \mathrm{~s}$ along the animal's trajectory. Cells were defined as head direction cells if the mean vector lengths of the recorded cells were larger than the $99^{\text {th }}$ percentile of the mean vector lengths in the shuffled distribution. Angular stability was computed by calculating the correlation of firing rates across directional bins generated from the first and second halves of the same trial. Head direction cells with angular stability lower than 0.3 were excluded for further analysis.

\section{Analysis of border cells}

Border or boundary vector cells were identified by calculating, for each recorded cell, 
the difference between the maximal length of any of the four walls touching on any single spatial firing field of the cell and the average distance of the firing field to the nearest wall, divided by the sum of those two values ${ }^{5,17,43}$. Border scores ranged from -1 for cells with perfect central firing fields to +1 for cells with firing fields that exactly line up with at least one entire wall. Firing fields were defined as summation of neighboring pixels with total firing rates higher than 0.3 times the cell's maximum firing rate that covered a total area of at least $200 \mathrm{~cm}^{2}$.

Border cell classification was verified in the same way as for place cells, head direction cells and grid cells. For each permutation trial, the whole sequence of spike trains was time-shifted along the animal's trajectory by a random period between $20 \mathrm{~s}$ and $20 \mathrm{~s}$ less than the length of the entire trial, with the end wrapped to the start of the trial. A spatial firing rate map was then obtained, and a border score was estimated.

The distribution of border scores was calculated for the entire set of permutation trials from all recorded cells, and the $99^{\text {th }}$ percentile was then determined. Cells were defined as border cells if the border score from the observed data was higher than the $99^{\text {th }}$ percentile for border scores in the entire distribution generated from the permutated data.

\section{Whisker trimming}

To test how the removal of another salient somatosensation mediate by vibrissae input impacts on FL/HL S1 spatial cells, we carefully trimmed all whiskers of each recoded rat bilaterally using blunt surgical scissors to within 1-2 mm above the skin surface just right before each recording session every day. Since bilateral whisker-trimmed rats did not exhibit abnormal behaviors, we continuously recorded somatosensory units from 2 whiskers-trimmed rats.

\section{Environmental manipulations}

For visual landmark rotation, we first recorded neuronal activity in the standard session followed by a $90^{\circ}$ cue-card rotation in the clockwise or counterclockwise direction. 
Then another standard session was performed with the cue-card rotated back to the original position. For recording in the elevated platform without walls, we first recorded the somatosensory spatial cells in the square box, followed by the recording in the elevated platform without walls. Finally, the animals were returned to the original square box for another recording session. For the recording of border cells in the presence of inserted wall, we first identified the somatosensory border cells in the square box. Then the recording session was followed by the insertion of a wall along the center of the external wall. Another recording session was performed after removing the inserted wall. For food/no food comparisons, we recorded the somatosensory spatial cells in two consecutive recording sessions without and with throwing food pellets into the running enclosure.

\section{Histology and reconstruction of recording positions}

At the end of the experiment, rats were euthanized with an overdose of sodium pentobarbital and perfused transcardially with phosphate-buffered saline (PBS) followed by $4 \%$ paraformaldehyde (PFA). Brains were removed and stored in 4\% PFA overnight. The brain was then placed in 10, 20 and 30\% sucrose/PFA solution sequentially across 72 hours before sectioning using a cyrotome. Thirty-micron sections were obtained through the implant region. Sections were mounted on glass slides and stained with cresyl violet (Sigma-Aldrich). The final recording positions were determined from digitized images of the Nissl-stained sections. Positions of each individual recordings were estimated from the deepest tetrode track, notes on tetrode advancement with tissue shrinkage correction by dividing the distance between the brain surface and electrode tips by the last advanced depth of the recording electrodes. Electrode traces were confirmed to be located within the hindlimb region (S1HL) from eight implanted rats, the forelimb region (S1FL) from one rat and the shoulder region (S1Sh) from another rat but all electrode tracking paths from 10 implanted rats were verified to be away from the barrel field (S1BF) of the primary somatosensory cortex according to the Rat Brain Atlas ${ }^{83}$. 
Data Availability: Recording dataset will be prepared available in a forthcoming public domain, and inquiries into acquiring the recording dataset beforehand should be directed to the corresponding author.

Acknowledgments: We would like to thank five anonymous reviewers for their insightful comments and constructive suggestions during the peer reviews. We are indebted to H. Wu, W. Tang, H. Chen, S. Lv, H. Yang and J. Ni for their encouragement and generous help. We would like to acknowledge J. Cai, B. Deng, L. Hu and S. Wang for their technical assistance. We would like to express our gratitude to Neil Burgess and Caswell Barry for sharing their Matlab codes with us. We would like to appreciate Calvin Young for his critical comments on the manuscript. X.L. is supported by the Chongqing Municipality postdoctoral fellowship (Grant\# cstc2019jcyj-bshX0035). We are grateful for two medical research funds from Xinqiao Hospital (Grant\# 2017A034 and Grant\# 2019XQY16) and a startup fund from Army Medical University (Grant\# 2017R028) to S.-J.Z. This work was also supported by the National Natural Science Foundation of China through the Project Grant NSFC-31872775 to S.-J.Z. Original results published in this manuscript were previously uploaded on the preprint server for biology (https://www.biorxiv.org/content/10.1101/473090v1) at bioRxiv on November $19,2018^{41}$.

Author Contributions: S.-J.Z. conceived and designed the study. X.L. and S.-J.Z. performed the experiments, collected the data and performed the analyses. X.L. made the figures. S.-J.Z. wrote the manuscript.

\section{Additional Information}

Supplementary information (supplementary Figs. S1-S33 and Table S1) accompanies this paper.

Competing Interests: The authors declare no competing interests. 
a
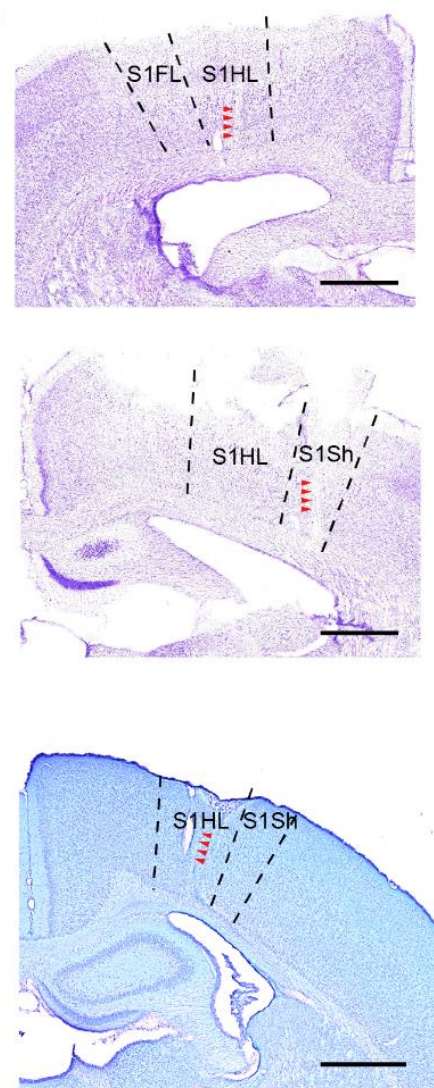
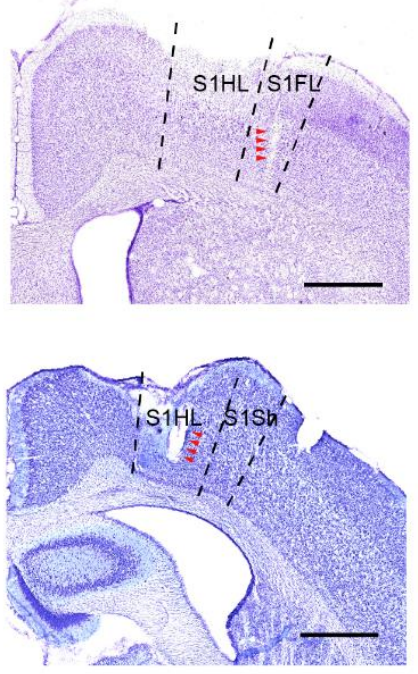

b

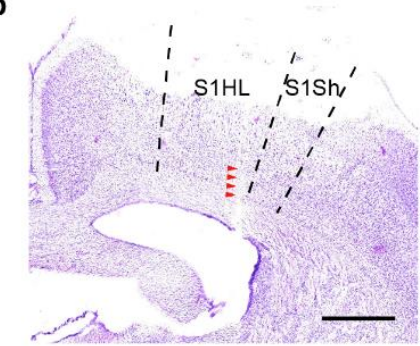

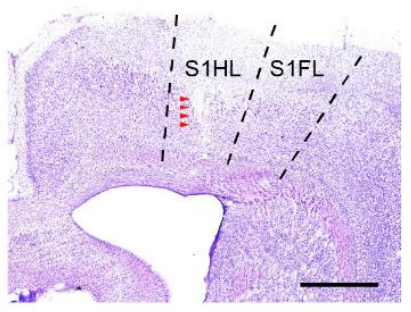
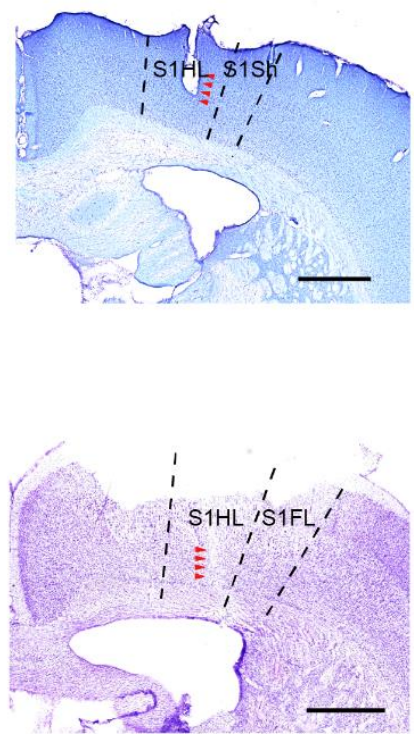

Fig. S1 Electrode track and recording locations in the primary somatosensory cortex.

(a) Cresyl violet-stained coronal brain sections show recording electrode tracks (arrowheads) and final recording positions in seven rats with tetrodes implanted in the rat primary somatosensory cortex. (b) Cresyl violet-stained coronal brain sections showing representative recording locations (arrowheads) for two additionally implanted rats in the whisker trimming experiment. Broken dashes depict the boundaries of the hindlimb region (S1HL), shoulder region (S1Sh) and forelimb (S1FL) region of the primary somatosensory cortex according to the rat brain atlas of Paxinos and Watson (2007). Scale bar, $1 \mathrm{~mm}$. 
a
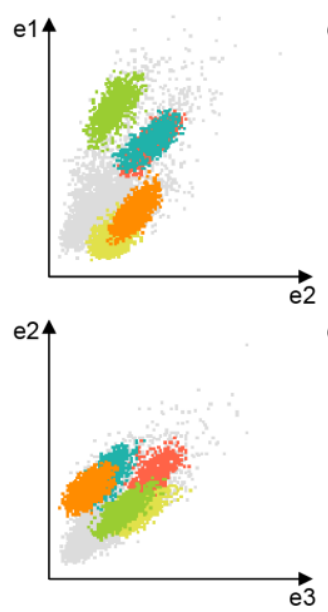

C
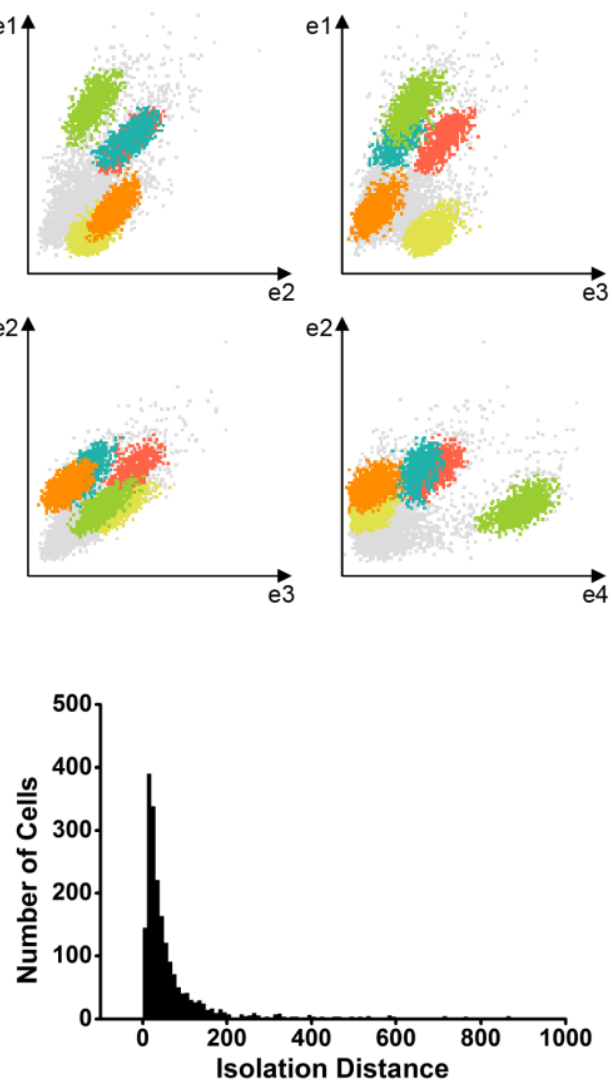

b
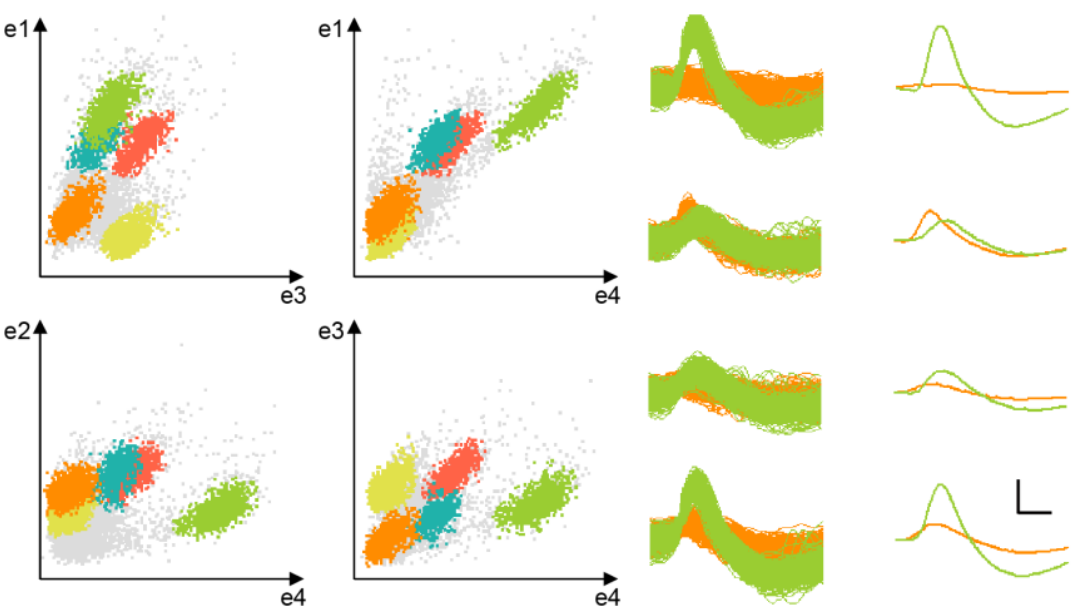

d

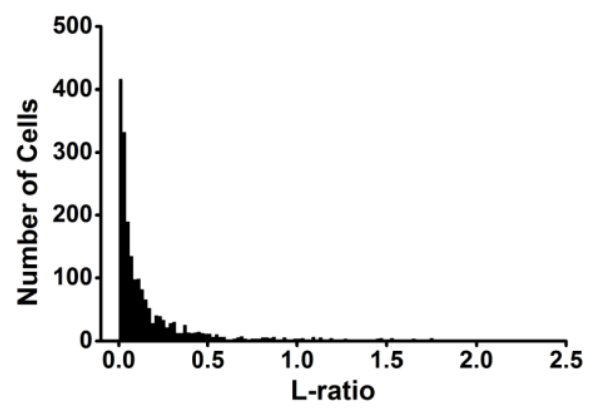

Fig. S2 Cluster diagrams, waveforms and isolation quality of spike clusters recorded from the somatosensory cortex.

(a) Scatterplots show the relationship between peak-to-trough amplitudes for all spikes for each wire (e1-e4) in a tetrode. (b) Overlaid and mean waveforms from two separated green and orange clusters in the scatterplots are shown for four electrodes from the same tetrode. Scale bar, $150 \mu \mathrm{V}, 200 \mu \mathrm{s}$. (c) The distribution of isolation distance for identified somatosensory units. (d) Same as (c) for the L-ratio. 
a
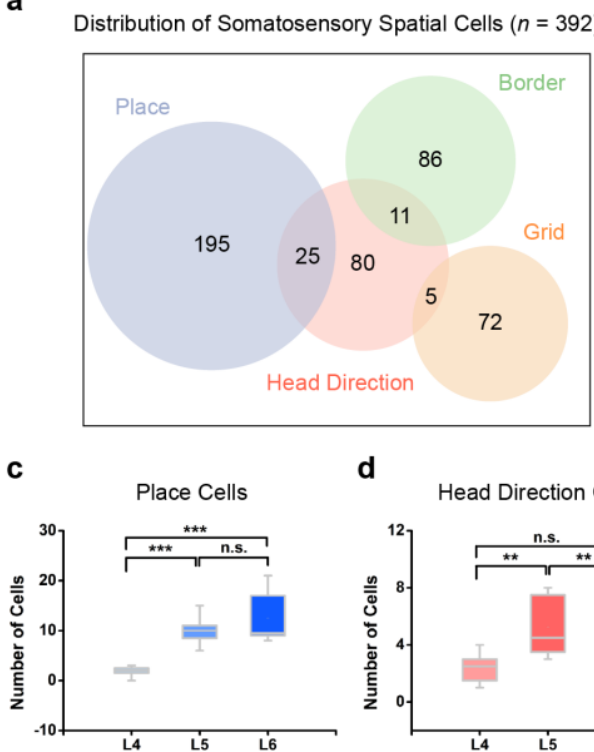

d

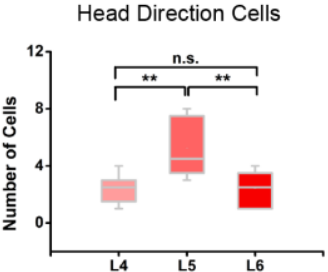

b
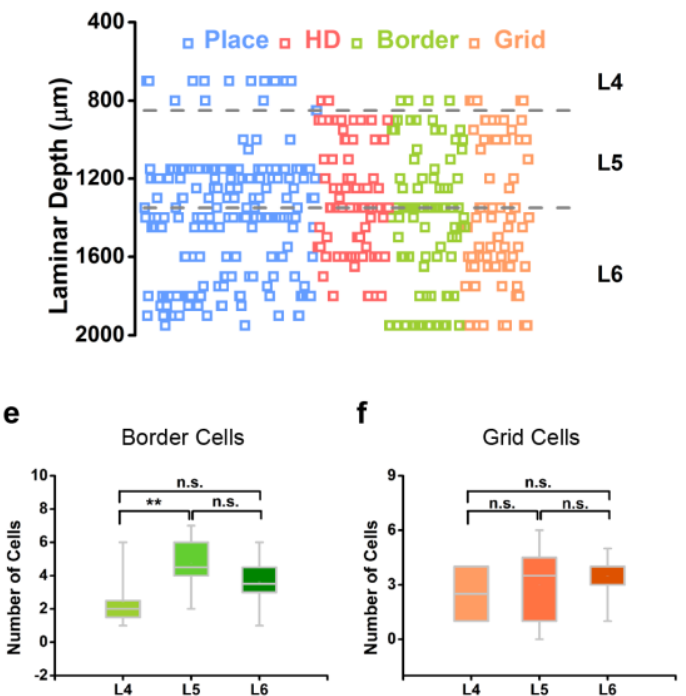
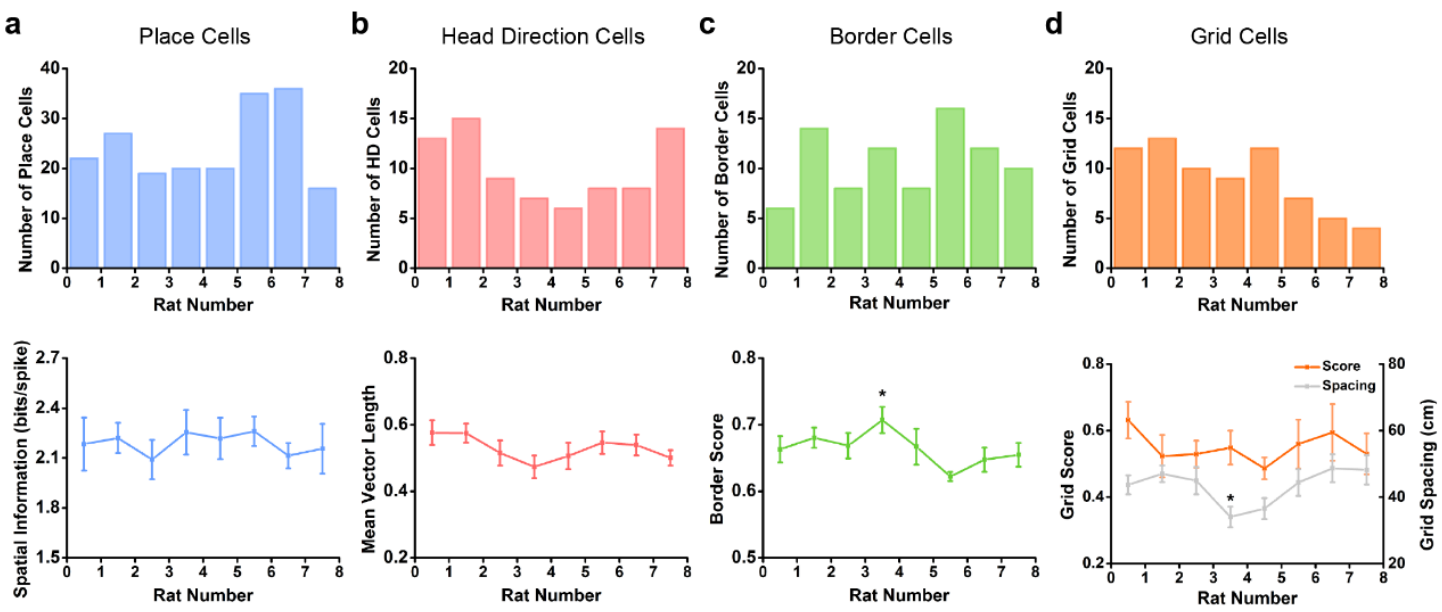

Fig. S4 Distribution of number and characteristics of four different somatosensory spatial cell types.

(a-d) Distribution of number (upper panels) and spatial characteristics (bottom panels) of identified somatosensory place cells (a), head direction cells (b), border cells (c) and grid cells (d) across different animals. 


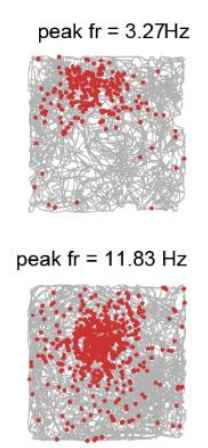

peak $\mathrm{fr}=4.59 \mathrm{~Hz}$

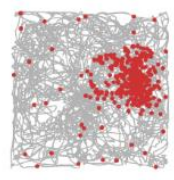

peak $\mathrm{fr}=8.69 \mathrm{~Hz}$

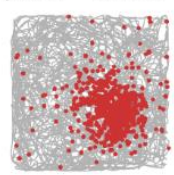

peak $\mathrm{fr}=10.68 \mathrm{~Hz}$

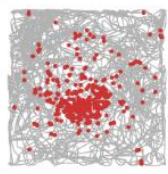

peak $\mathrm{fr}=20.00 \mathrm{~Hz}$

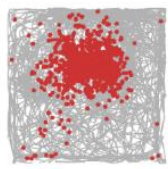

peak $\mathrm{fr}=4.87 \mathrm{~Hz}$

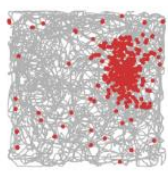

peak $\mathrm{fr}=11.03 \mathrm{~Hz}$

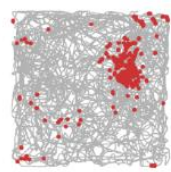

peak $\mathrm{fr}=16.48 \mathrm{~Hz}$

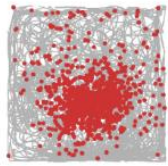

peak $\mathrm{fr}=5.32 \mathrm{~Hz}$

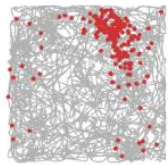

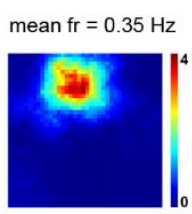

mean $\mathrm{fr}=0.88$

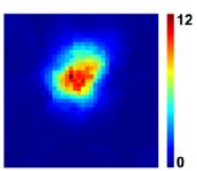

mean $\mathrm{fr}=0.51 \mathrm{~Hz}$

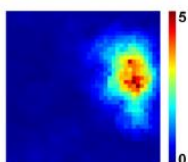

mean $\mathrm{fr}=0.83 \mathrm{~Hz}$

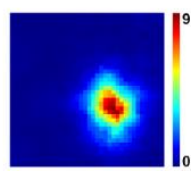

mean $\mathrm{fr}=0.60 \mathrm{~Hz}$
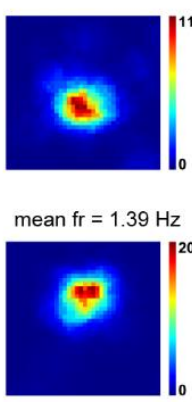

mean $\mathrm{fr}=0.43 \mathrm{~Hz}$

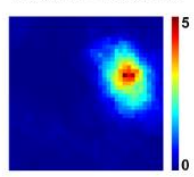

mean $\mathrm{fr}=0.72 \mathrm{~Hz}$

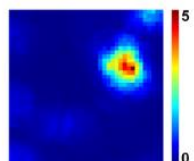

mean $\mathrm{fr}=1.26 \mathrm{~Hz}$

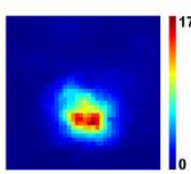

mean $\mathrm{fr}=0.31 \mathrm{~Hz}$

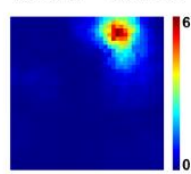

si $=1.85$

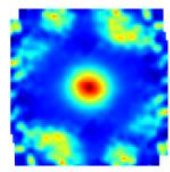

si $=1.77$
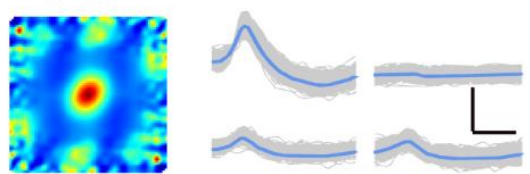

$\mathrm{si}=1.80$
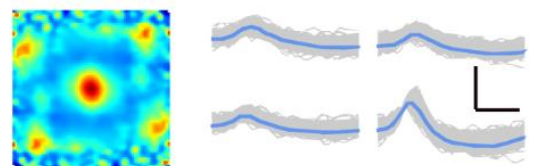

$\mathrm{si}=1.73$
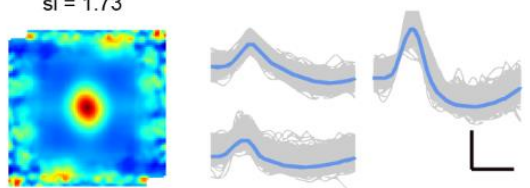

$\mathrm{si}=1.84$
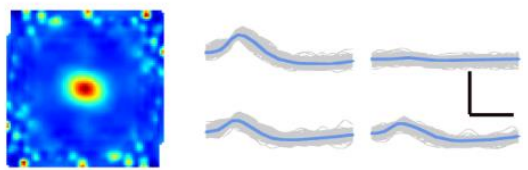

$\mathrm{si}=1.73$
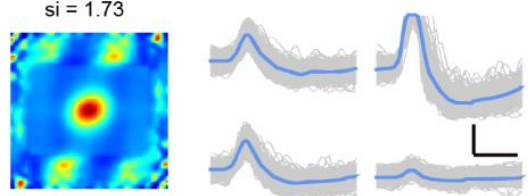

si $=1.77$
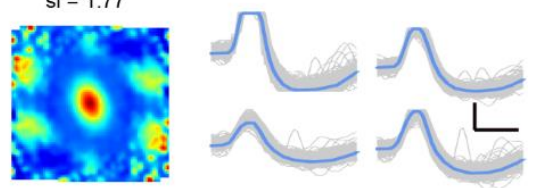

$\mathrm{si}=2.00$
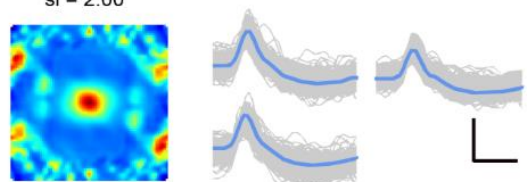

$\mathrm{si}=1.71$
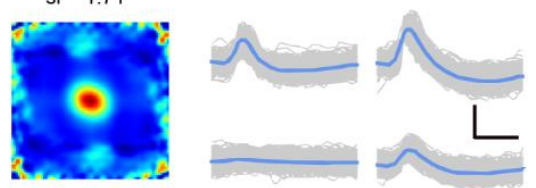

$\mathrm{si}=2.34$
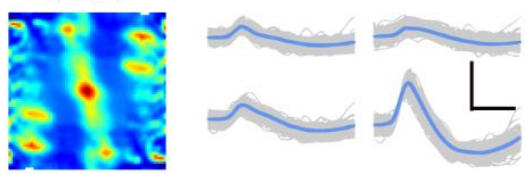

Fig. S5 More examples of somatosensory place cells recorded from the 


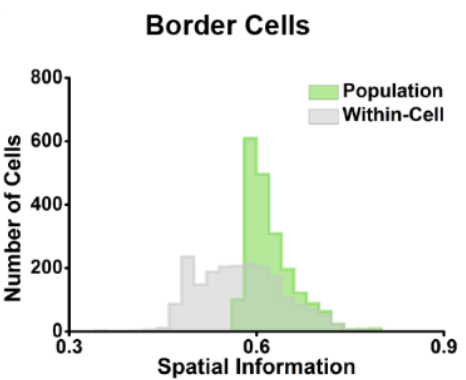

b

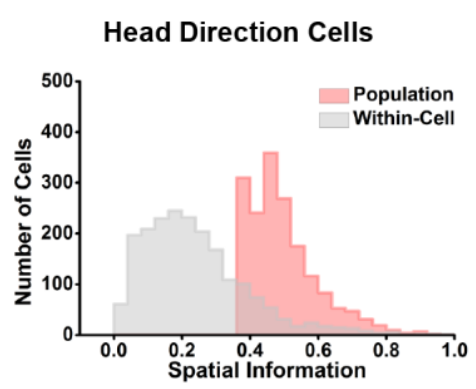

d

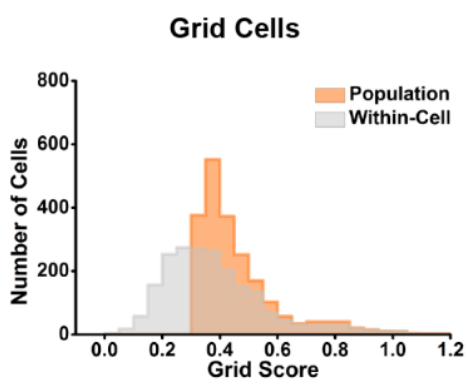

873 Fig. S6 Comparison of spatial threshold defined by population shuffling and 874 within-cell shuffling for four different somatosensory spatial cell types.

875 (a-d) Histograms showing the $99^{\text {th }}$ percentile significance level of each randomly 876 shuffled distribution for population shuffling and within-cell shuffling of all 2025 877 identified single units for identified somatosensory place cells (a), head direction cells 878 (b), border cells (c) and grid cells (d). 
a
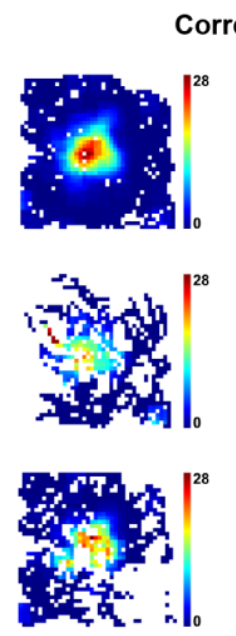

b
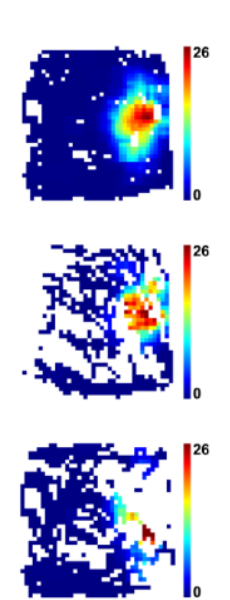

C
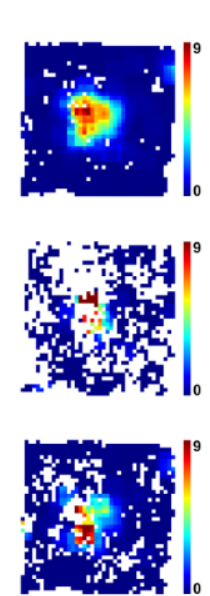

Corrected
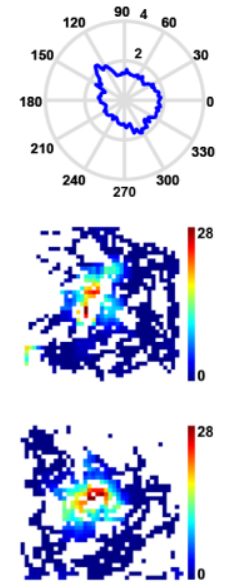

Corrected
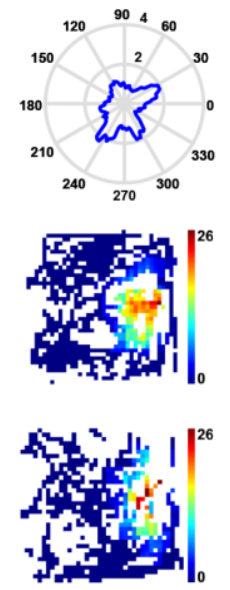

Corrected
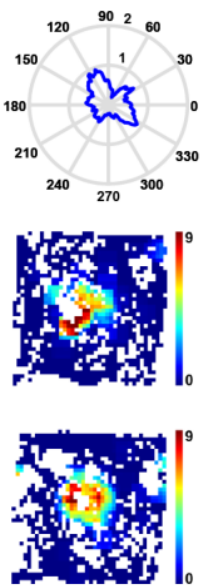

Uncorrected
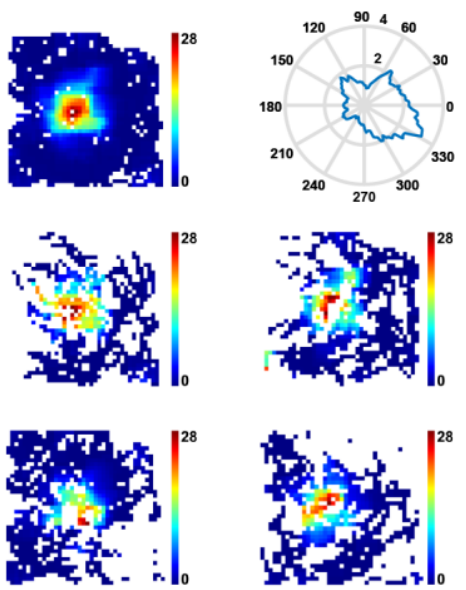

Uncorrected
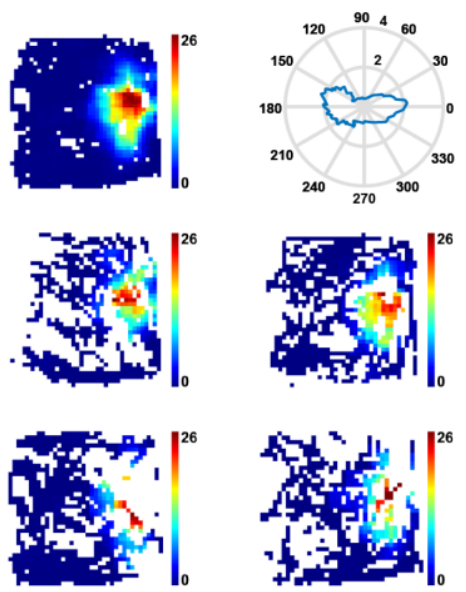

Uncorrected
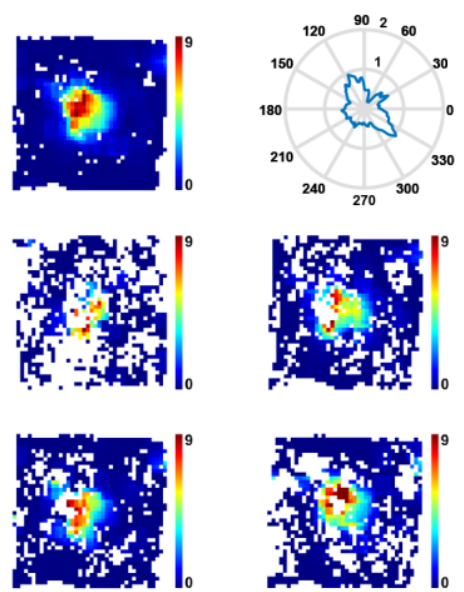

Fig. S7 Quantification of spatial responses of somatosensory place cells using the maximum likelihood factorial model.

882 (a-c) Firing rate maps of three representative place cells from Fig. 1b. Left column

883 shows the corrected locational and directional firing rate maps using the maximum-

884 likelihood correction approach; right column shows the corresponding uncorrected 
885 firing rate maps. Spatial firing rate maps in four directions are shown in the lower panels.

886 Note the similar locational responses in four different directions.

a
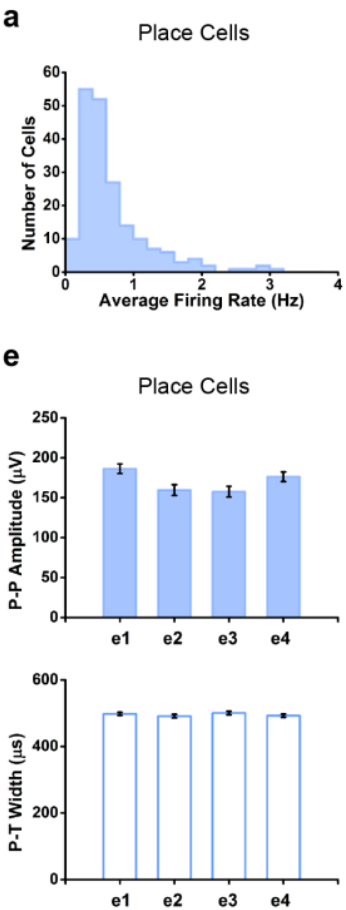

b

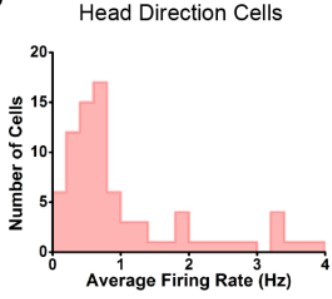

f
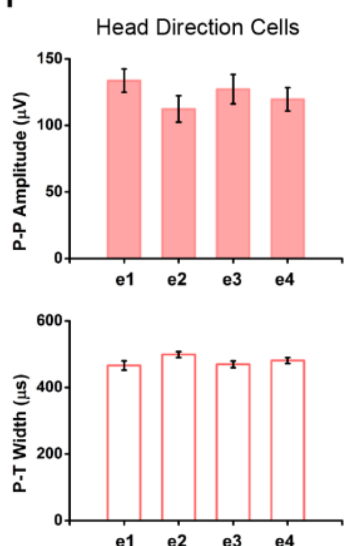

c

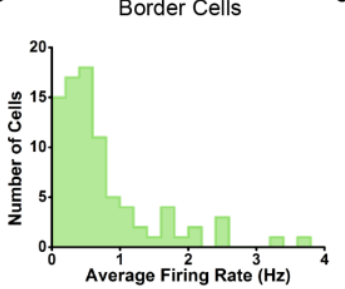

g
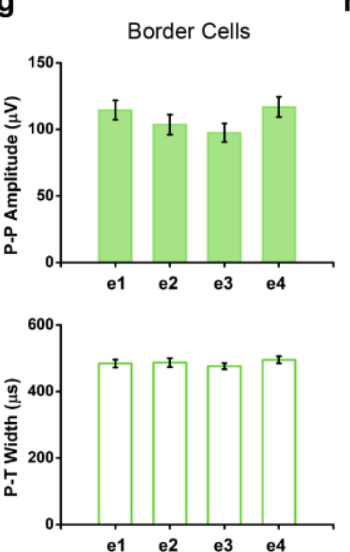

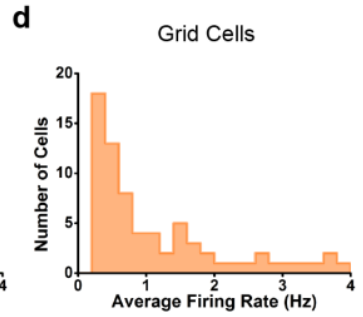

h
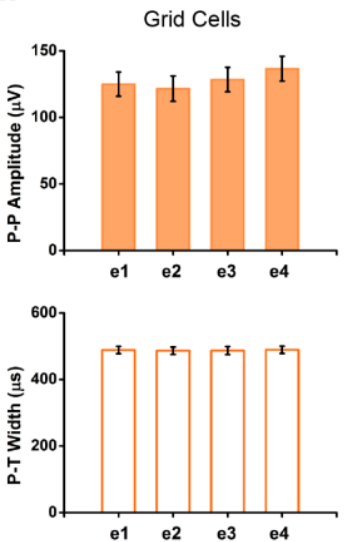

887

Fig. S8 Distribution of the average firing rate and summary of spike waveform for

\section{four different somatosensory spatial cell types.}

(a-d) Histograms showing the average firing rate of identified somatosensory place cells (a), head direction cells (b), border cells (c) and grid cells (d). (e-h) Histograms showing the peak-to-peak amplitudes (upper panels) and peak to trough width (bottom panels) of spike waveforms on four electrodes of identified somatosensory place cells (e), head direction cells (f), border cells (g) and grid cells (h). 
a

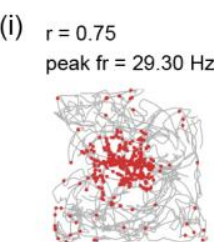

(ii)

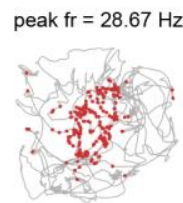

C

(i) $r=0.60$

peak $\mathrm{fr}=6.25 \mathrm{~Hz}$

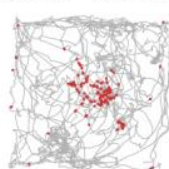

(ii)

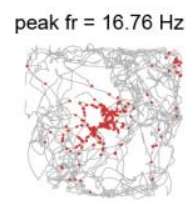

First Half

mean $\mathrm{fr}=1.47 \mathrm{~Hz}$

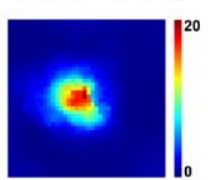

Second Half

mean $\mathrm{fr}=1.14 \mathrm{~Hz}$

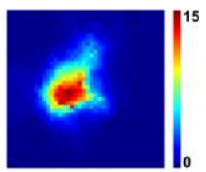

First Half

mean $\mathrm{fr}=0.31 \mathrm{~Hz}$

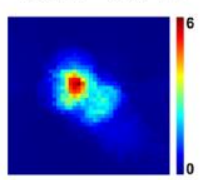

Second Half

mean $\mathrm{fr}=0.65 \mathrm{~Hz}$

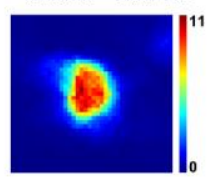

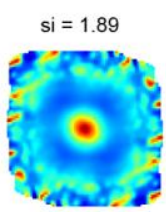

$\mathrm{si}=1.83$

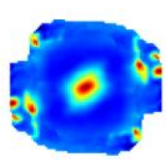

$\mathrm{si}=1.75$

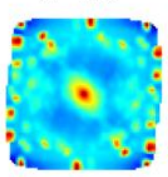

$s i=2.46$

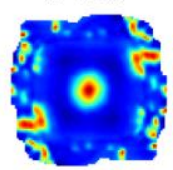

b

(i) $r=0.78$

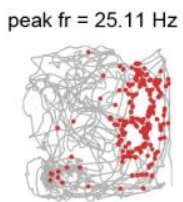

(ii)

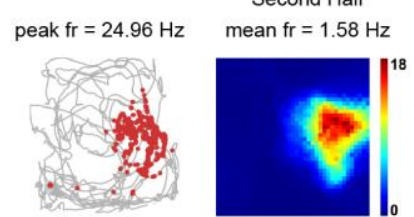

$\mathrm{si}=2.29$

d

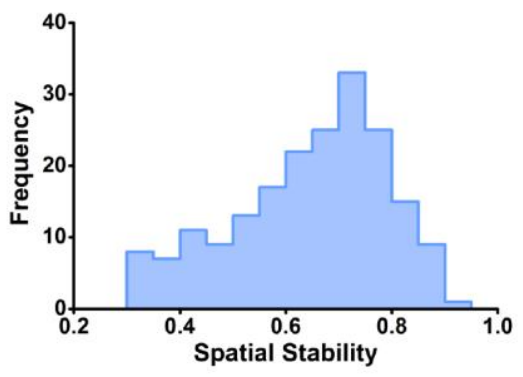

895

896

897

898

899

900

901

902

903

904

905

906

907

Fig. S9 Spatial stability of somatosensory place cells.

(a-c) Intra-trial spatial stability between the first and second halves of three representative place cells from Fig. 1b. Trajectory (grey line) with superimposed spike locations (red dots) (left column); rate maps (middle column) and autocorrelation maps (right column) for the first half (i) and the second half (ii) of the trials. Firing rate is color-coded with blue indicating minimum firing rate and red indicating maximum firing rate. The scale of the autocorrelation maps is twice that of the spatial firing rate maps. Peak firing rate (fr), mean firing rate (fr) and spatial information (si) are labelled at the top of the plots. Pearson's correlation coefficients of firing rate maps between the first and second halves are indicated with $r$ at the top-left corner. (d) Distribution of spatial stability of firing rate maps between the first and second halves of all identified somatosensory place cells. 
a

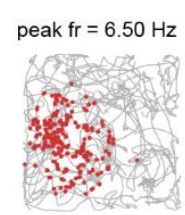

peak $\mathrm{fr}=4.62 \mathrm{~Hz}$

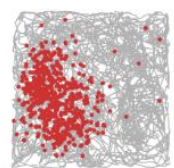

b
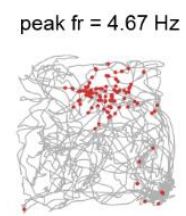

peak $\mathrm{fr}=9.81 \mathrm{~Hz}$

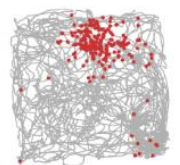

C

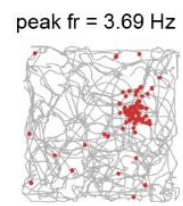

peak $\mathrm{fr}=4.87 \mathrm{~Hz}$

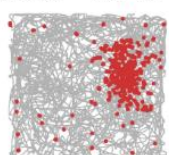

10 Minutes

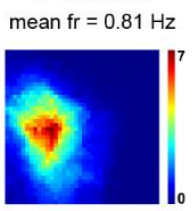

30 Minutes

mean $\mathrm{fr}=0.69 \mathrm{~Hz}$

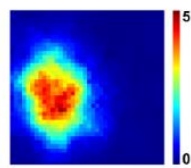

10 Minutes

mean fr $=0.14 \mathrm{~Hz}$

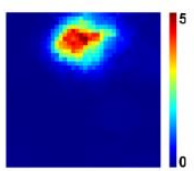

30 Minutes

mean $\mathrm{fr}=0.31 \mathrm{~Hz}$

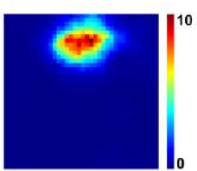

10 Minutes

mean $\mathrm{fr}=0.28 \mathrm{~Hz}$

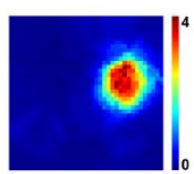

30 Minutes

mean $\mathrm{fr}=0.43 \mathrm{~Hz}$

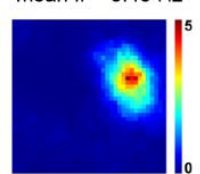

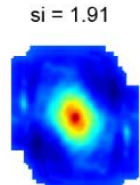

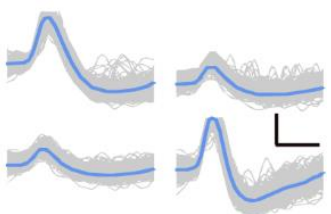

si $=1.93$
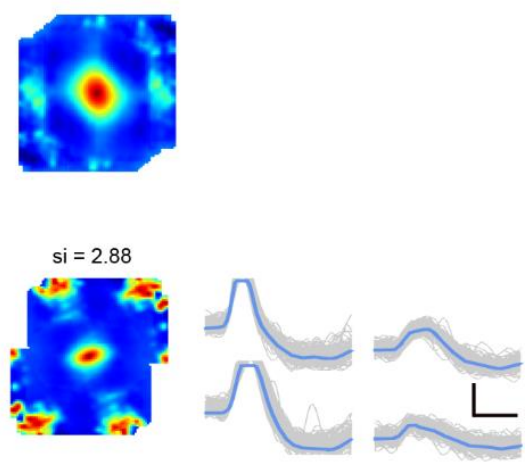

$\mathrm{si}=3.13$

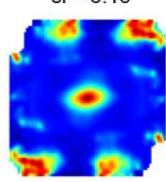

$\mathrm{si}=1.64$
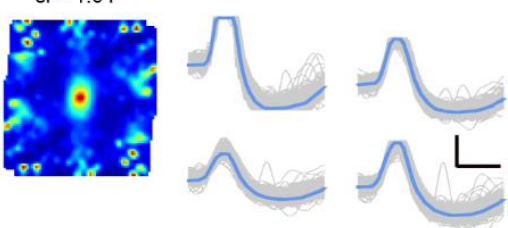

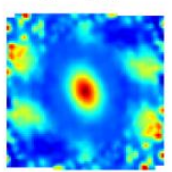

Fig. S10 Persistence of somatosensory place fields from longer recording sessions.

(a-c) Spatial stability of three representative somatosensory place cells between the short (top panels) and longer (bottom panels) recording sessions. Trajectory (grey line) with superimposed spike locations (red dots) (left column); spatial firing rate maps

913 (middle column) and autocorrelation diagrams (right column). Firing rate is color-

914 coded with blue indicating minimum firing rate and red indicating maximum firing rate.

915 The scale of the autocorrelation maps is twice that of the spatial firing rate maps. Peak 916 firing rate (fr), mean firing rate (fr) and spatial information (si) for each recording 917 session are labelled at the top of the panels. Spike waveforms on four electrodes are 918 shown on the right column. Scale bar, $150 \mu \mathrm{V}, 300 \mu \mathrm{s}$. 
a
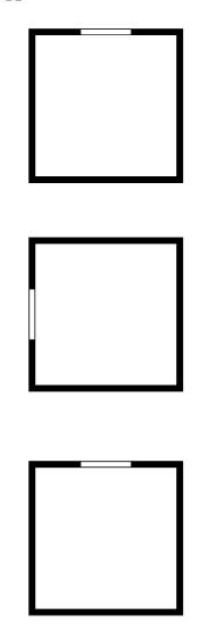

b
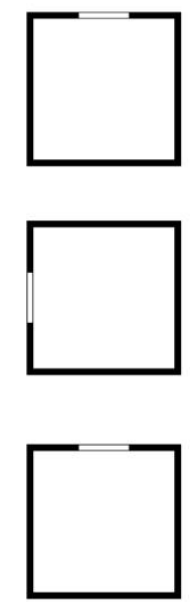

C
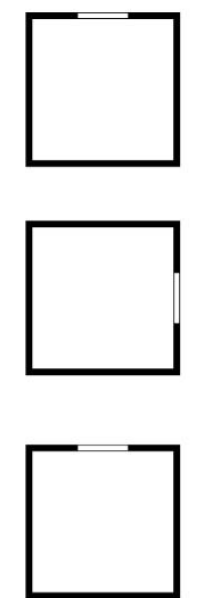

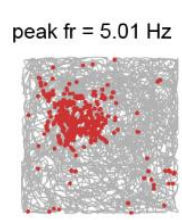

peak $\mathrm{fr}=7.81 \mathrm{~Hz}$

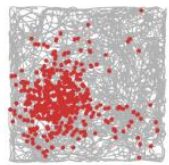

peak $\mathrm{fr}=6.06 \mathrm{~Hz}$

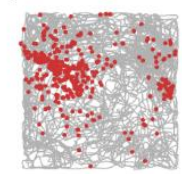

peak $\mathrm{fr}=5.01 \mathrm{~Hz}$

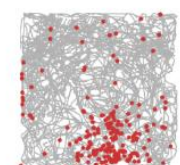

peak $\mathrm{fr}=7.81 \mathrm{~Hz}$

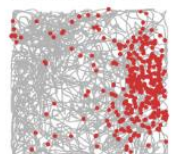

peak $\mathrm{fr}=6.06 \mathrm{~Hz}$

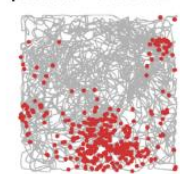

peak $\mathrm{fr}=5.01 \mathrm{~Hz}$

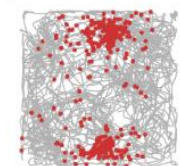

peak $\mathrm{fr}=10.40 \mathrm{~Hz}$

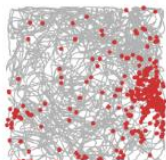

peak $\mathrm{fr}=6.53 \mathrm{~Hz}$

pon $\mathrm{Wr}$

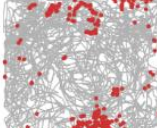

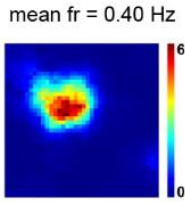

mean $\mathrm{fr}=0.50 \mathrm{~Hz}$

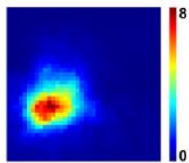

mean $\mathrm{fr}=10.35 \mathrm{~Hz}$
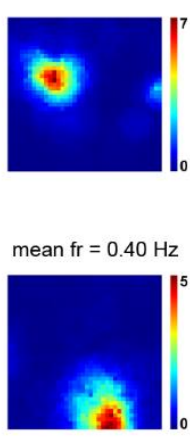

mean $\mathrm{fr}=0.50 \mathrm{~Hz}$

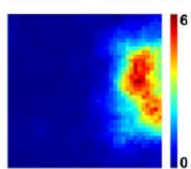

mean $\mathrm{fr}=10.35 \mathrm{~Hz}$
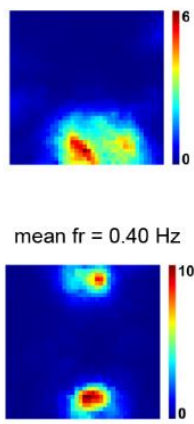

mean $\mathrm{fr}=0.46 \mathrm{~Hz}$

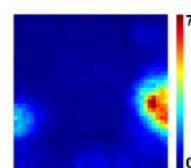

mean $\mathrm{fr}=0.49 \mathrm{~Hz}$

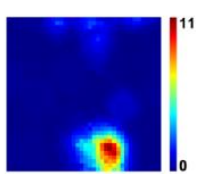

$\mathrm{si}=2.14$
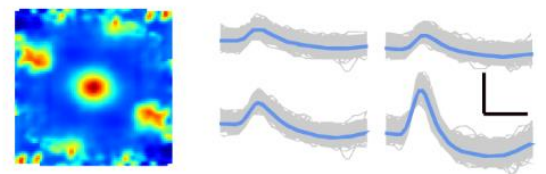

si $=1.81$

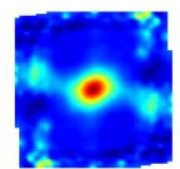

$\mathrm{si}=1.39$

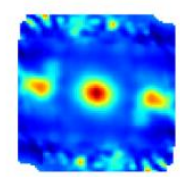

$\mathrm{si}=2.14$

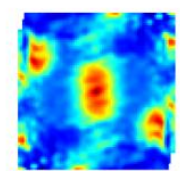

$\mathrm{si}=1.81$

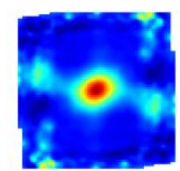

$\mathrm{si}=1.39$
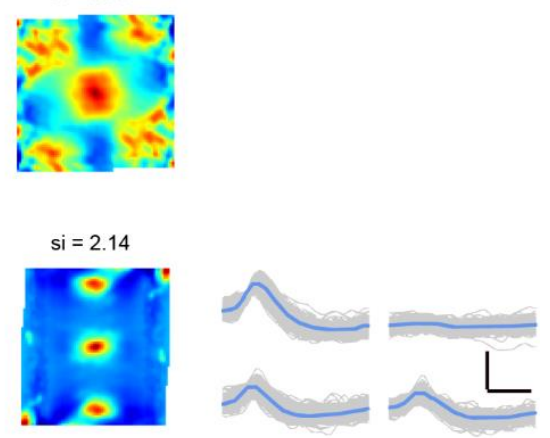

Fig. S11 Cue-rotation control of somatosensory place cell coding.

921 (a-c) Each panel shows the response of the same somatosensory place cell during three

922 sessions of the cue-rotation condition. Top panels, before the cue-rotation; middle

923 panels, counterclockwise or clockwise $90^{\circ}$ of cue-rotation; bottom panels, cue-rotation 
924 back to the original condition. The cue card is represented by a white arc in each panel.

925 The experimental diagram (left column); trajectory (grey line) with superimposed spike

926 locations (red dots) (middle left column); spatial firing rate maps (middle right column)

927 and autocorrelation diagrams (right column). Firing rate is color-coded with blue

928 indicating minimum firing rate and red indicating maximum firing rate. The scale of

929 the autocorrelation maps is twice that of the spatial firing rate maps. Peak firing rate

930 (fr), mean firing rate (fr) and spatial information (si) for each recording session are

931 labelled at the top of the panels. Spike waveforms on four electrodes are shown on the

932 right column. Scale bar, $150 \mu \mathrm{V}, 300 \mu \mathrm{s}$. 
a
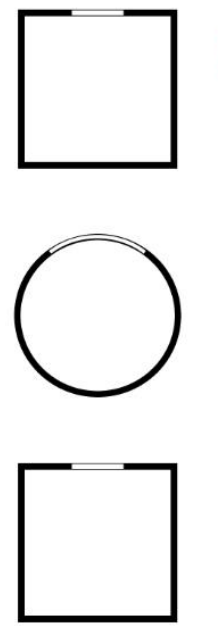

b
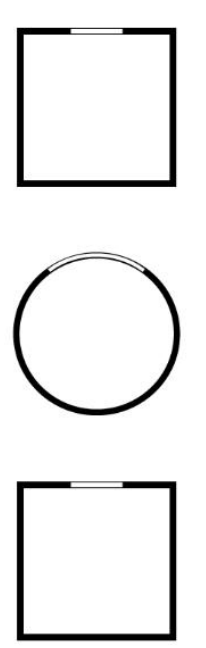

C
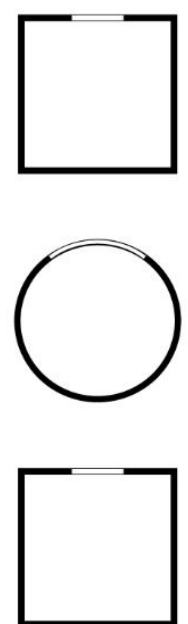

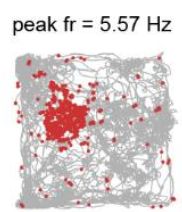

peak $\mathrm{fr}=2.75 \mathrm{~Hz}$

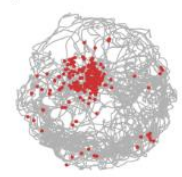

peak $\mathrm{fr}=5.03 \mathrm{~Hz}$

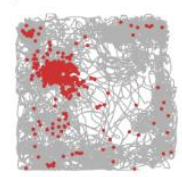

peak $\mathrm{fr}=9.00 \mathrm{~Hz}$

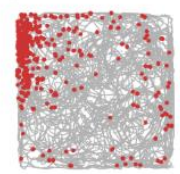

peak $\mathrm{fr}=1.74 \mathrm{~Hz}$

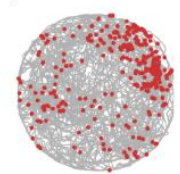

peak $\mathrm{fr}=6.87 \mathrm{~Hz}$

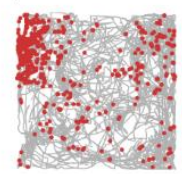

peak $\mathrm{fr}=6.07 \mathrm{~Hz}$

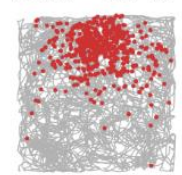

peak $\mathrm{fr}=1.53 \mathrm{~Hz}$

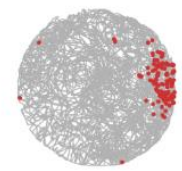

peak $\mathrm{fr}=5.23 \mathrm{~Hz}$

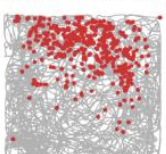

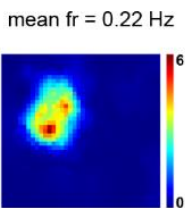
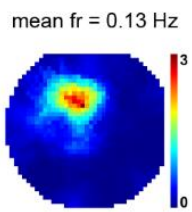

mean $\mathrm{fr}=0.23 \mathrm{~Hz}$
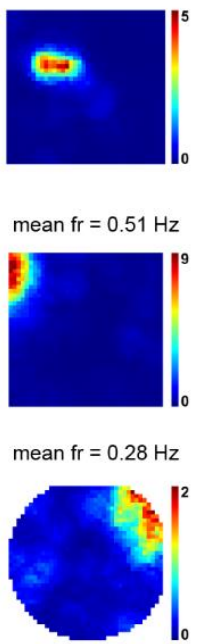

mean $\mathrm{fr}=0.51 \mathrm{~Hz}$
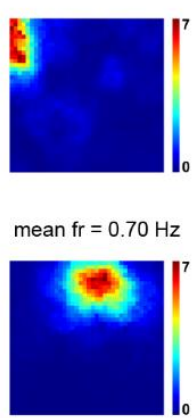

mean $\mathrm{fr}=0.13 \mathrm{~Hz}$

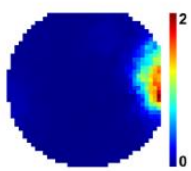

mean $\mathrm{fr}=0.57 \mathrm{~Hz}$

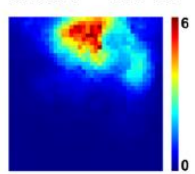

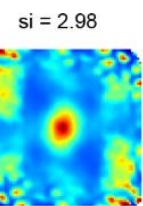
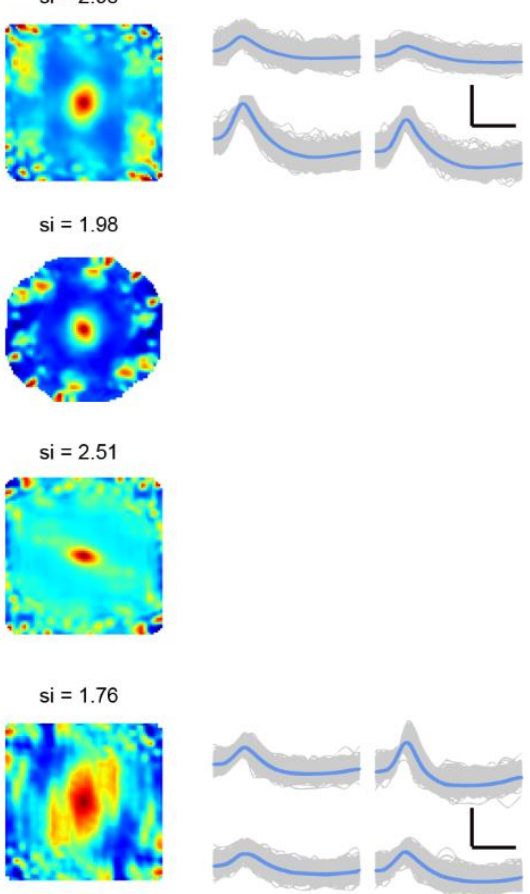

si $=0.66$

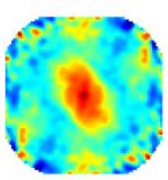

si $=1.24$

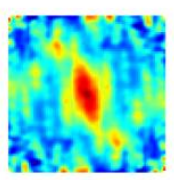

$\mathrm{si}=2.28$
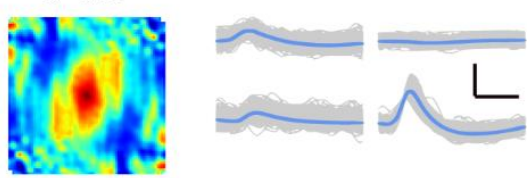

$\mathrm{si}=2.20$

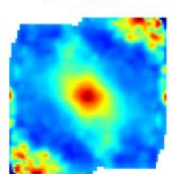

935 (a-c) Spatial responses of three representative somatosensory place cells in different

936 boxes in a constant location. The experimental diagram (left column); trajectory (grey

937 line) with superimposed spike locations (red dots) (middle left column); spatial firing 
rate maps (middle right column) and autocorrelation diagrams (right column). Firing rate is color-coded with blue indicating minimum firing rate and red indicating maximum firing rate. The scale of the autocorrelation maps is twice that of the spatial 941 firing rate maps. Peak firing rate (fr), mean firing rate (fr) and spatial information (si) 942 for each recording session are labelled at the top of the panels. Spike waveforms on 943 four electrodes are shown on the right column. Scale bar, $150 \mu \mathrm{V}, 300 \mu \mathrm{s}$.

a

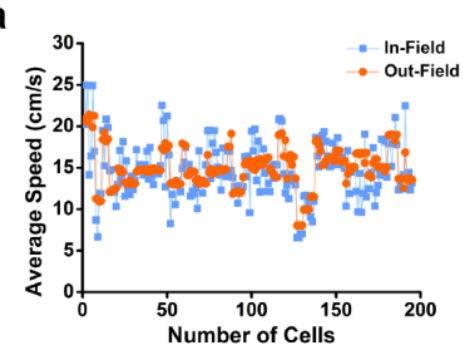

b

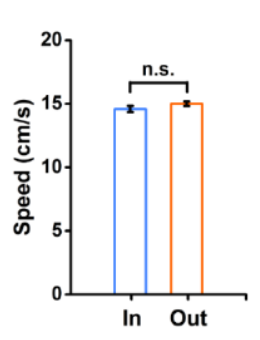

945 Fig. S13 Distribution of the average in-field and out-field running speed for place 946 cells in the somatosensory cortex.

947 (a) The distribution of the average running speed within and outside the firing fields of 948 all identified place cells in the somatosensory cortex. (b) The comparison of the average 949 in-field and out-field running speed. $n=195, P=0.10$, two-tailed paired $t$-test, n.s., not 950 significant. 
a
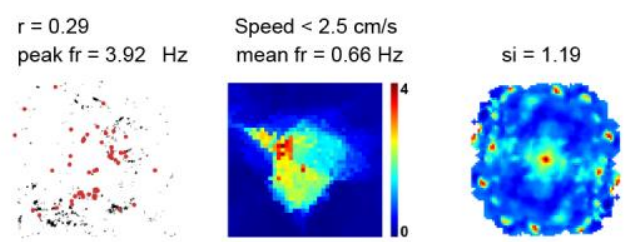

peak $\mathrm{fr}=19.82 \mathrm{~Hz}$

Speed $>2.5 \mathrm{~cm} / \mathrm{s}$
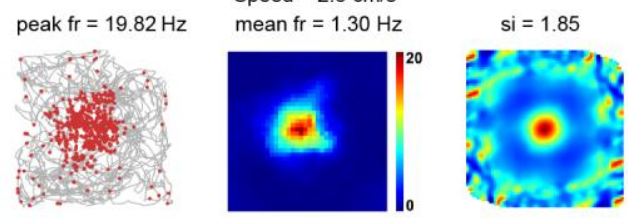

b

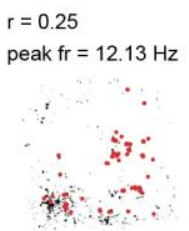

Speed $<2.5 \mathrm{~cm} / \mathrm{s}$

mean $\mathrm{fr}=0.87 \mathrm{~Hz}$
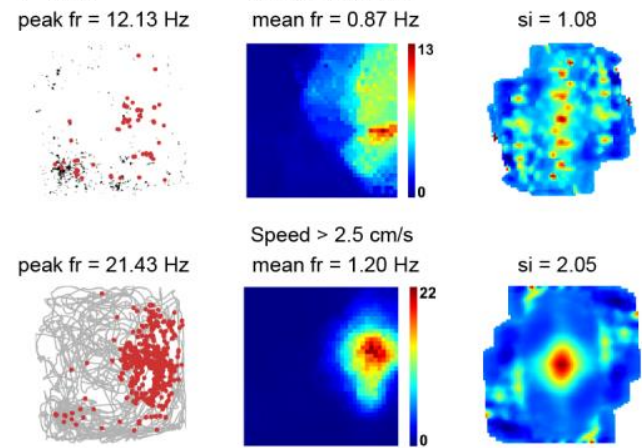

Speed $>2.5 \mathrm{~cm} / \mathrm{s}$

mean $\mathrm{fr}=1.20 \mathrm{~Hz}$

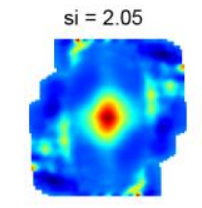

C $\quad r=0.51$

peak $\mathrm{fr}=8.12 \mathrm{~Hz}$
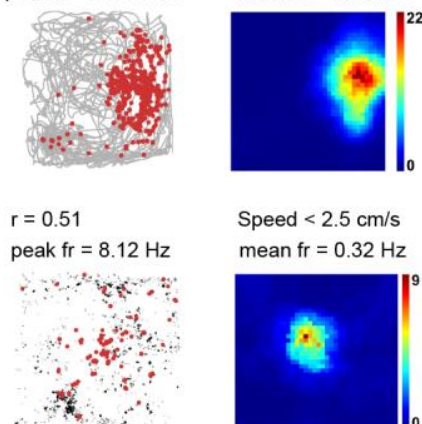

Speed $<2.5 \mathrm{~cm} / \mathrm{s}$

mean $\mathrm{fr}=0.32 \mathrm{~Hz}$
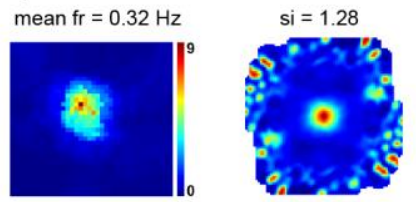

Speed $>2.5 \mathrm{~cm} / \mathrm{s}$
mean $\mathrm{fr}=0.48 \mathrm{~Hz}$

peak $\mathrm{fr}=9.45 \mathrm{~Hz}$
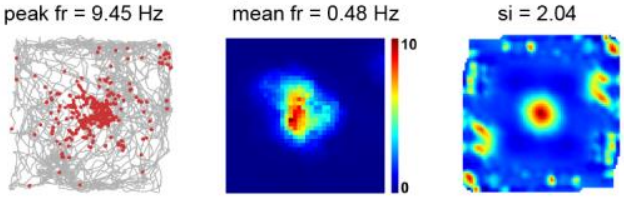

Fig. S14 Comparison of the spatial response of somatosensory place cells between active running and slow mobility.

(a-c) Comparison of spatial response of representative place cells from Fig. 1b with instantaneous running speeds $<2.5 \mathrm{~cm} / \mathrm{s}$ and instantaneous running speeds $>2.5 \mathrm{~cm} / \mathrm{s}$, respectively. Trajectory (grey line) with superimposed spike locations (red dots) (left column); rate maps (middle column) and autocorrelation maps (right column) for instantaneous running speeds $<2.5 \mathrm{~cm} / \mathrm{s}$ and instantaneous running speeds $>2.5 \mathrm{~cm} / \mathrm{s}$, respectively. Firing rate is color-coded with blue indicating minimum firing rate and red indicating maximum firing rate. The scale of the autocorrelation maps is twice that of the spatial firing rate maps. Peak firing rate (fr), mean firing rate (fr) and spatial 
bioRxiv preprint doi: https://doi.org/10.1101/473090; this version posted October 26, 2020. The copyright holder for this preprint (which was not certified by peer review) is the author/funder, who has granted bioRxiv a license to display the preprint in perpetuity. It is made available under aCC-BY-NC-ND 4.0 International license.

962 information (si) are labelled at the top of the plots. Pearson's correlation coefficients

963 between two firing rate maps when the instantaneous running speeds $<2.5 \mathrm{~cm} / \mathrm{s}$ and

964 instantaneous running speeds $>2.5 \mathrm{~cm} / \mathrm{s}$ are indicated with $r$ at the top-left corner. 

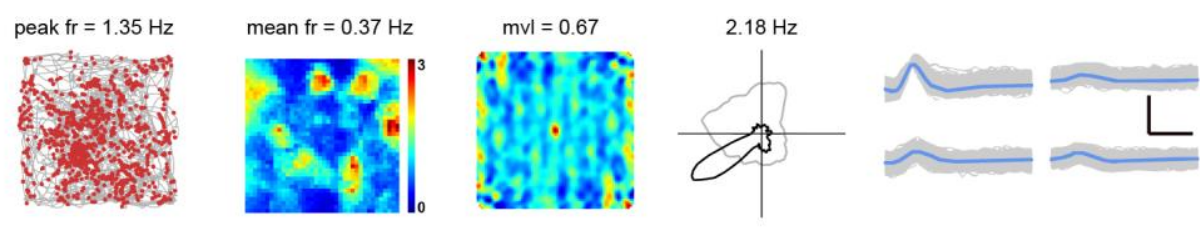

peak $\mathrm{fr}=8.83 \mathrm{~Hz}$
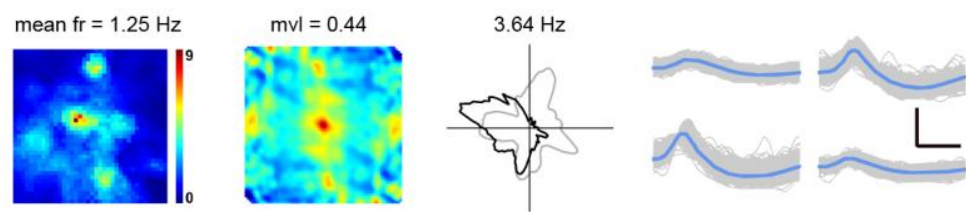

peak $\mathrm{fr}=2.41 \mathrm{~Hz}$
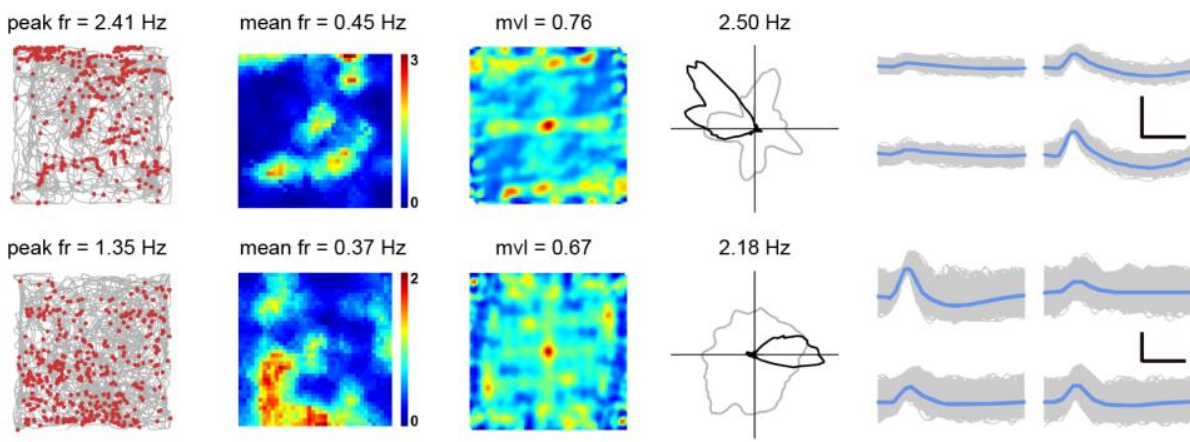

peak $\mathrm{fr}=5.58 \mathrm{~Hz}$
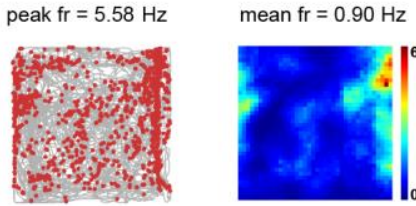

$\mathrm{mvl}=0.66$
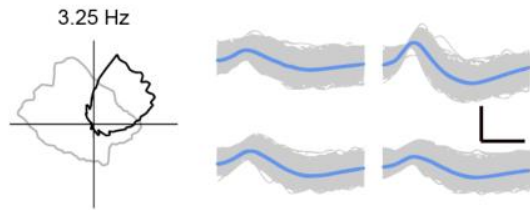

peak $\mathrm{fr}=7.89 \mathrm{~Hz}$

mean $\mathrm{fr}=1.53 \mathrm{~Hz}$
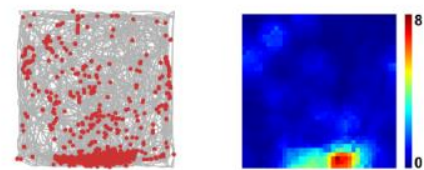

$\mathrm{mvl}=0.51$
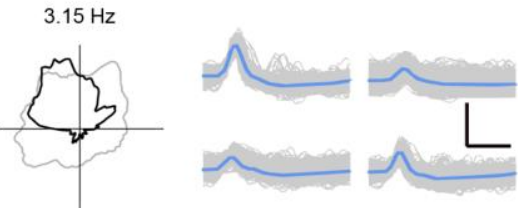

peak $\mathrm{fr}=2.12 \mathrm{~Hz}$

mean $\mathrm{fr}=0.57 \mathrm{~Hz}$

$\mathrm{mvl}=0.41$
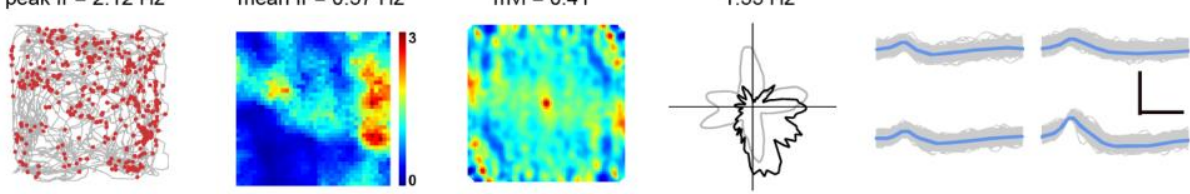

peak $\mathrm{fr}=11.78 \mathrm{~Hz}$

mean $\mathrm{fr}=1.78 \mathrm{~Hz}$
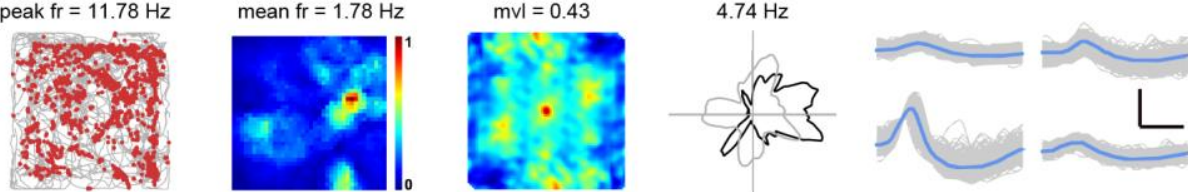

peak $\mathrm{fr}=7.91 \mathrm{~Hz}$

mean $\mathrm{fr}=1.90 \mathrm{~Hz}$

$\mathrm{mvl}=0.47$
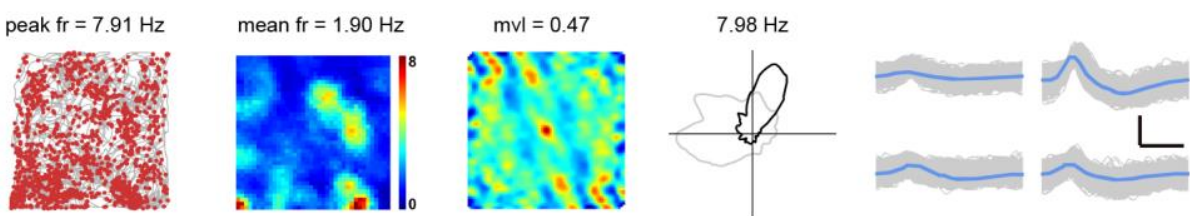

Deak $\mathrm{fr}=1.95 \mathrm{~Hz}$
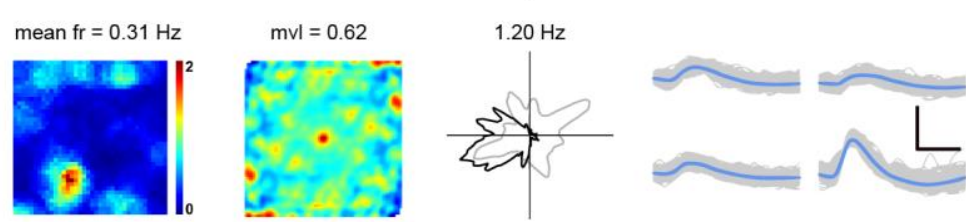
968 (a-c) Representative somatosensory head direction cells with bin coverage over 90\%.

969 Trajectory (grey line) with superimposed spike locations (red dots) (left column);

970 spatial firing rate maps (middle left column), autocorrelation diagrams (middle right

971 column) and head direction tuning curves (black) plotted against dwell-time polar plot

972 (grey) (right column). Firing rate is color-coded with dark blue indicating minimal

973 firing rate and dark red indicating maximal firing rate. The scale of the autocorrelation

974 maps is twice that of the spatial firing rate maps. Peak firing rate (fr), mean firing rate

975 (fr), mean vector length (mvl) and angular peak rate for each representative head

976 direction cell are labelled at the top of the panels. The directional plots show strong

977 head direction tuning. Spike waveforms on four electrodes are shown on the right

978 column. Scale bar, $150 \mu \mathrm{V}, 300 \mu \mathrm{s}$. 
a
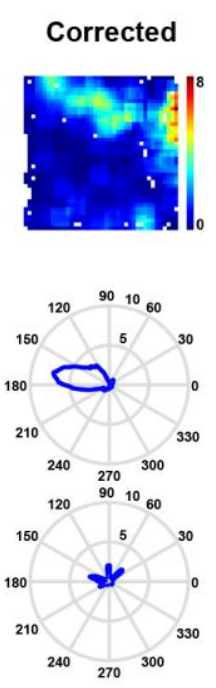

b
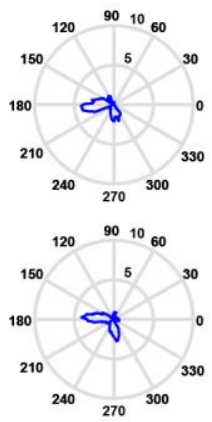

C
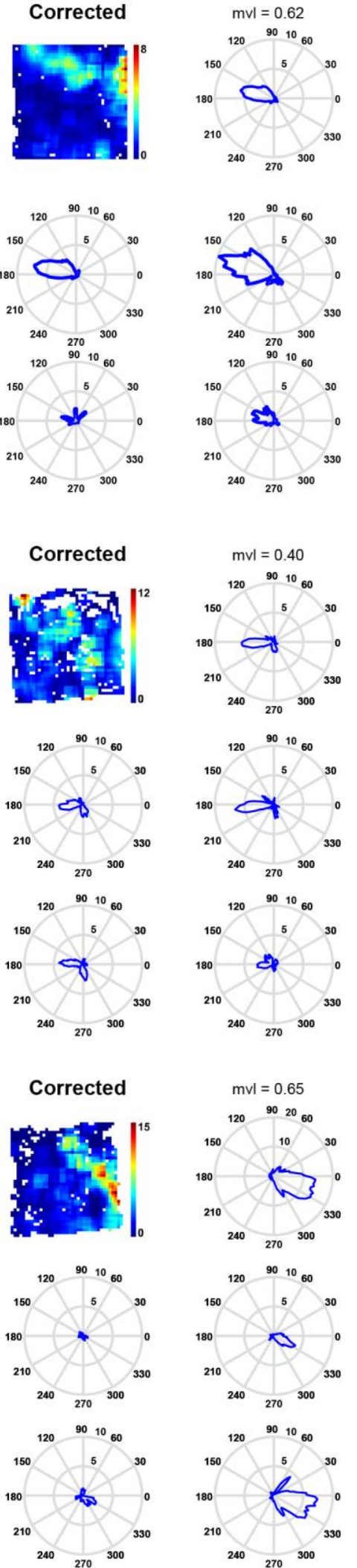

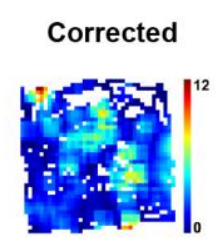

Fig. S16 Quantification of head direction selectivity of somatosensory head

\section{direction cells using the maximum likelihood factorial model.}
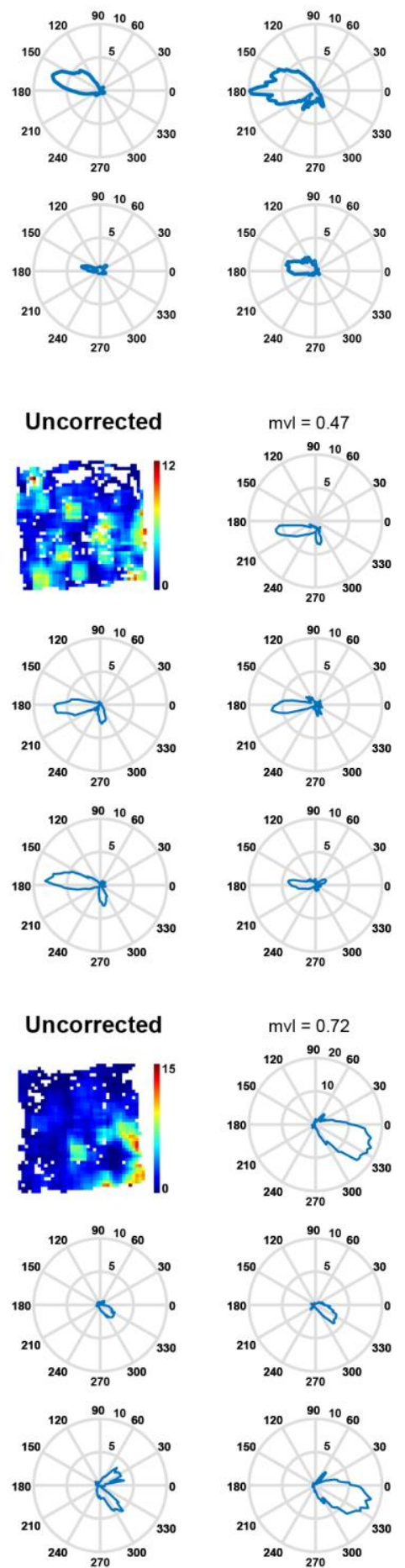

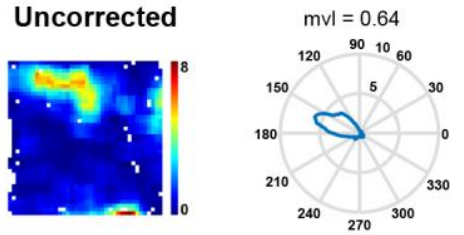

982 (a-c) Comparison of head directionality of three representative head direction cells from 
983 Fig. 2a using a maximum-likelihood approach. Top left two panels show the corrected

984

985

986

987

988

989

990

991

(ii)

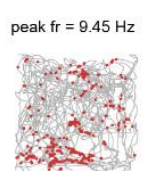

C

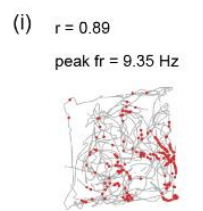

(ii)

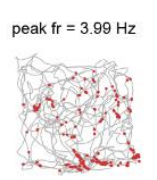

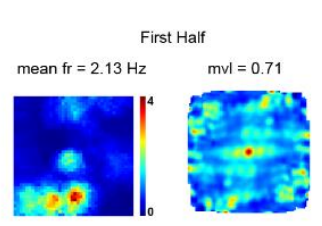
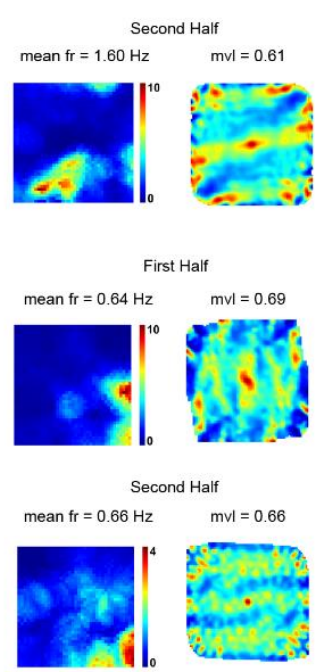
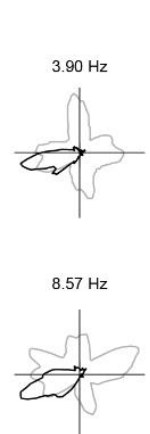

d
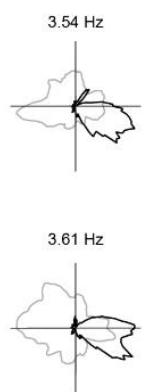

(ii)

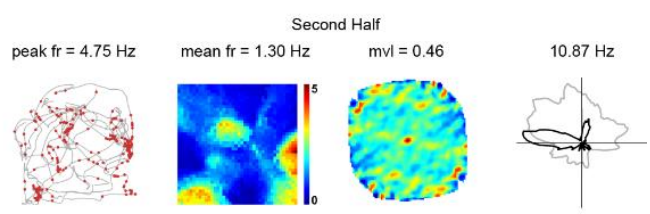

b (i) $r=0.98$
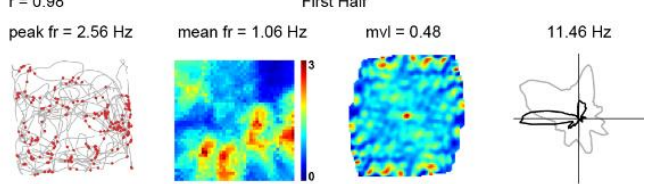

(i)
992

993

994

995

996

997

998

999

1000

1001

Fig. S17 Angular stability of somatosensory head direction cells.

(a-c) Intra-trial angular stability between the first and second halves of three representative head direction cells from Fig. 2a. Trajectory (grey line) with superimposed spike locations (red dots) (left column); spatial firing rate maps (middle left column), autocorrelation diagrams (middle right column) and head direction tuning curves (black) plotted against dwell-time polar plot (grey) (right column) for the first half (i) and the second half (ii) of the trials. Firing rate is color-coded with blue indicating minimum firing rate and red indicating maximum firing rate. The scale of the autocorrelation maps is twice that of the spatial firing rate maps. Peak firing rate (fr), mean firing rate (fr), mean vector length (mvl) and angular peak rate for each 
1002 representative head direction cell are labelled at the top of the panels. Correlation 1003 coefficients of the distributed firing rate across all directional bins between the first and 1004 second halves of individual recording trials are indicated with $r$. (d) Population 1005 histogram of angular stability of the distributed firing rate across all directional bins between the first and second halves of all identified somatosensory head direction cells. 
a
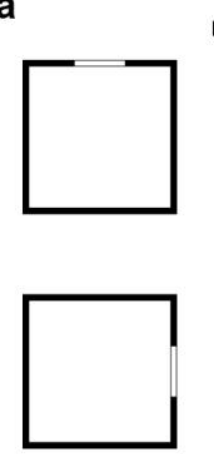

peak $\mathrm{fr}=2.56 \mathrm{~Hz}$
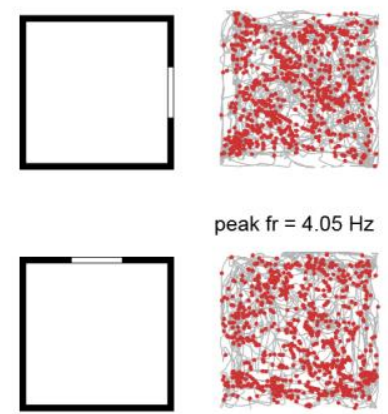

peak $\mathrm{fr}=4.05 \mathrm{~Hz}$

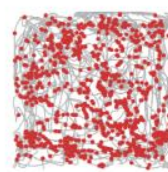

b

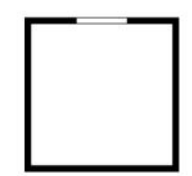

peak $\mathrm{fr}=10.52 \mathrm{~Hz}$
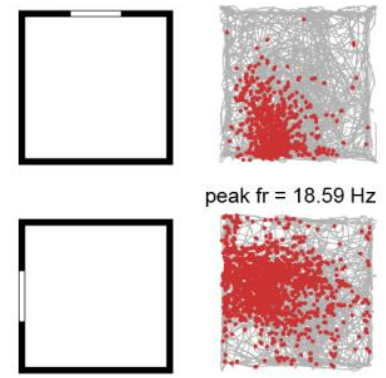

peak $\mathrm{fr}=18.59 \mathrm{~Hz}$

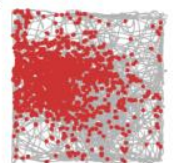

peak $\mathrm{fr}=8.69 \mathrm{~Hz}$

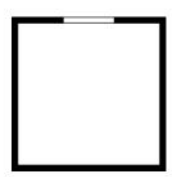

C

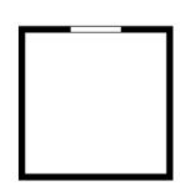

peak $\mathrm{fr}=4.35 \mathrm{~Hz}$

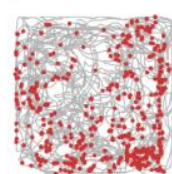

peak $\mathrm{fr}=1.44 \mathrm{~Hz}$

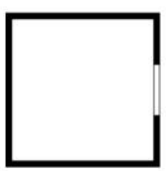

ins:

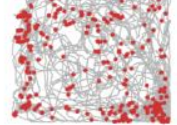

peak $\mathrm{fr}=1.23 \mathrm{~Hz}$
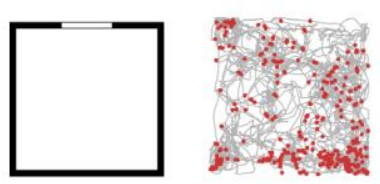

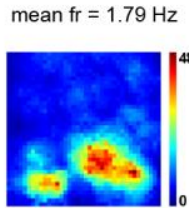

mean $\mathrm{fr}=0.88 \mathrm{~Hz}$

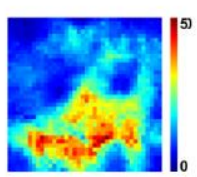

mean $\mathrm{fr}=1.07 \mathrm{~Hz}$
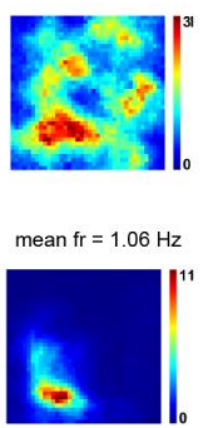

mean $\mathrm{fr}=2.35 \mathrm{~Hz}$

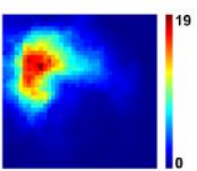

mean $\mathrm{fr}=1.03 \mathrm{~Hz}$

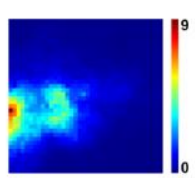

mean $\mathrm{fr}=0.80 \mathrm{~Hz}$
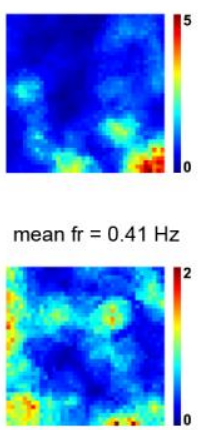

mean $\mathrm{fr}=0.30 \mathrm{~Hz}$

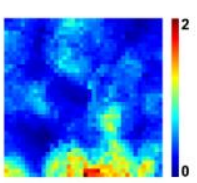

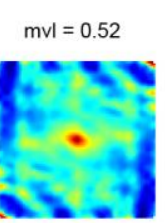
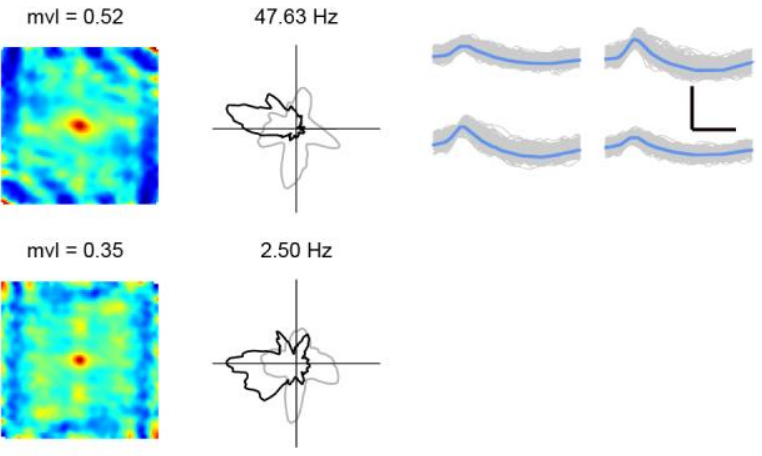

$\mathrm{mvl}=0.21$
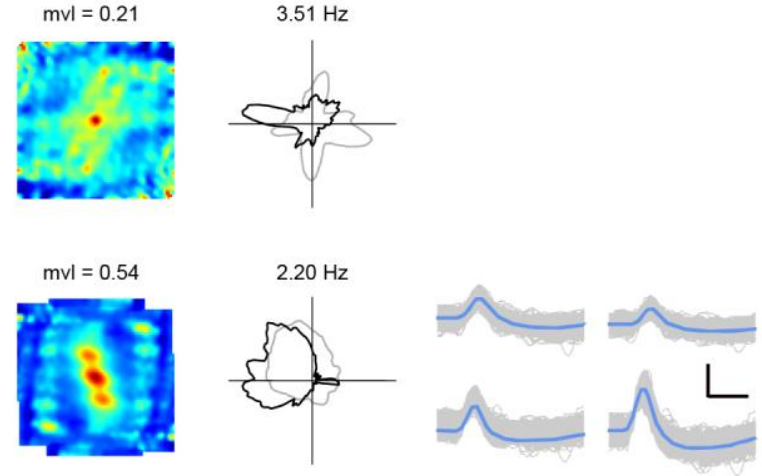
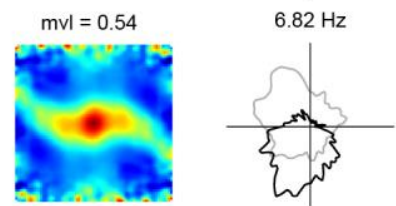

$\mathrm{mvl}=0.35$
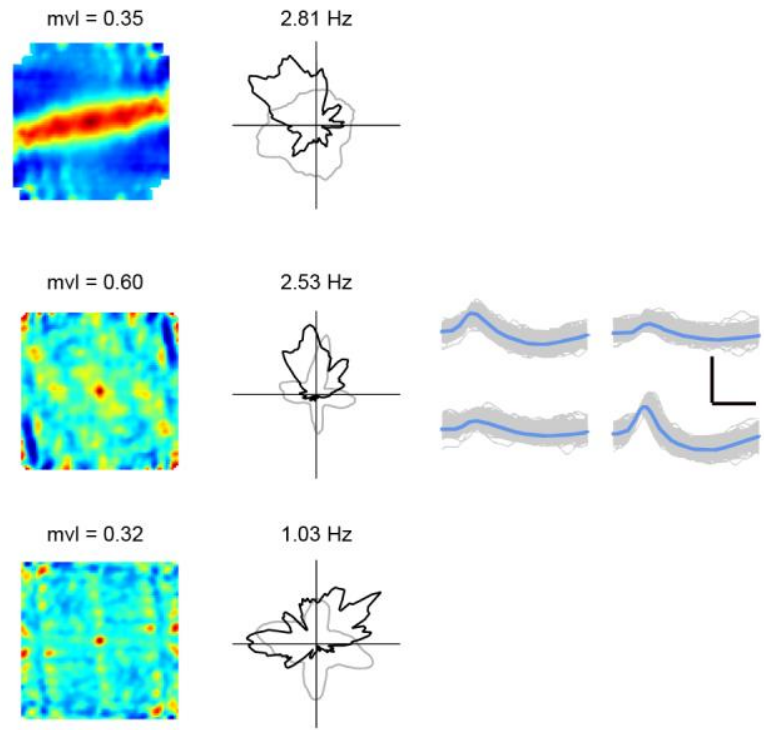

$$
b=0.43
$$
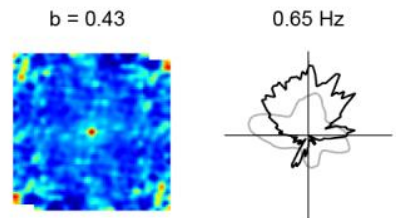

Fig. S18 Cue-rotation control of somatosensory head directional responses.

(a-c) Spatial responses of three representative somatosensory head direction cells 
during cue-rotation. Each panel shows the response of the same S1 head direction cell during three recording trails during the cue-rotation condition. Top panels, before the cue-rotation (a); middle panels, clockwise $90^{\circ}$ of cue-rotation (b); bottom panels, cuerotation back to the original condition (c). The cue card is represented by a white line in each panel. The experimental diagram (left column); trajectory (grey line) with superimposed spike locations (red dots) (middle left column); spatial firing rate maps (middle column), autocorrelation diagrams (middle right column) and head direction tuning curves (black) plotted against dwell-time polar plot (grey) (right column) for each recording trial. Firing rate is color-coded with blue indicating minimum firing rate and red indicating maximum firing rate. The scale of the autocorrelation maps is twice that of the spatial firing rate maps. Peak firing rate (fr), mean firing rate (fr), mean vector length (mvl) and angular peak rate for each representative head direction cell are labelled at the top of the panels. Spike waveforms on four electrodes are shown on the right column. Scale bar, $150 \mu \mathrm{V}, 300 \mu \mathrm{s}$.
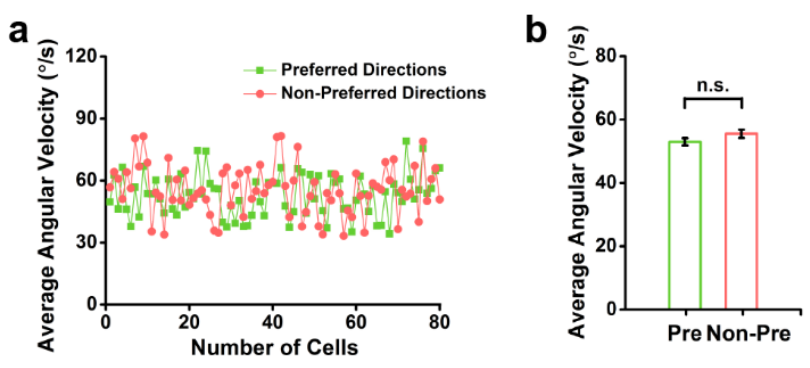

Fig. S19 Distribution of the average angular velocity in the preferred and nonpreferred firing directions for head direction cells in the somatosensory cortex.

(a) The distribution of the average angular velocity in the "preferred firing directions" and "non-preferred firing directions" for all identified head direction cells in the somatosensory cortex. (b) The comparison of the angular velocity in the "preferred firing directions" and "non-preferred firing directions". $n=80, P=0.12$, two-tailed paired $t$-test, n.s., not significant. 
No Food Pellets
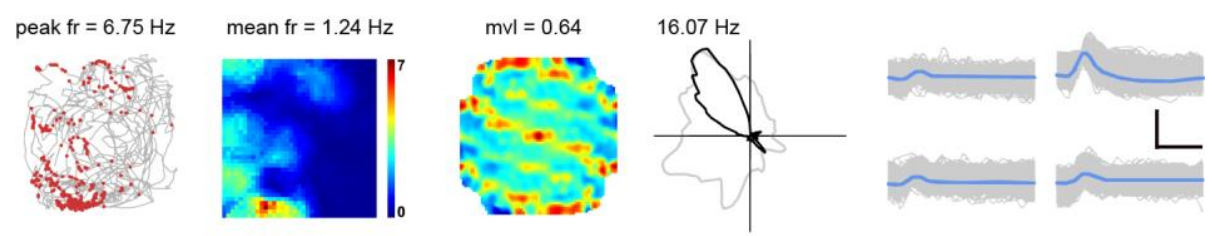

Food Pellets
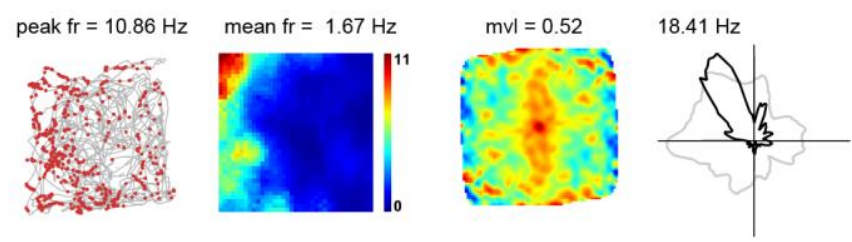

b Place Cell

No Food Pellets
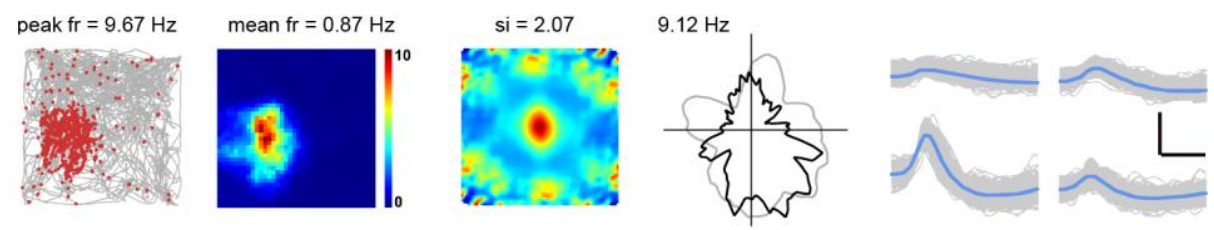

Food Pellets
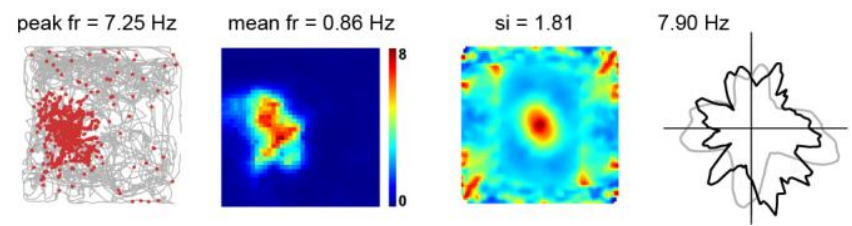

Fig. S20 Somatosensory place and head direction cells in the presence or absence of food pellets.

(a) The directional tuning of the same S1 head direction cell recorded with (top panels) and without (bottom panels) food pellets. (b) The firing fields of the same S1 place cell recorded with (top panels) and without (bottom panels) food pellets. Trajectory (grey line) with superimposed spike locations (red dots) (left column); spatial firing rate maps (middle left column), autocorrelation diagrams (middle right column) and head direction tuning curves (black) plotted against dwell-time polar plot (grey) (right column). Firing rate is color-coded with blue indicating minimum firing rate and red indicating maximum firing rate. The scale of the autocorrelation maps is twice that of the spatial firing rate maps. Peak firing rate (fr), mean firing rate (fr), mean vector length (mvl) or spatial information (si) and angular peak rate for each representative head direction cell are labelled at the top of the panels. Spike waveforms on four electrodes are shown on the right column. Scale bar, $150 \mu \mathrm{V}, 300 \mu \mathrm{s}$. 
a

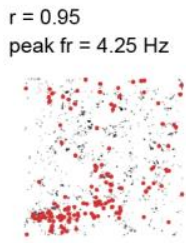

peak $\mathrm{fr}=7.58 \mathrm{~Hz}$

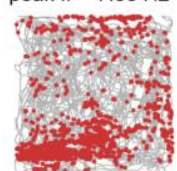

b

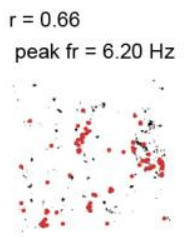

peak $\mathrm{fr}=4.17 \mathrm{~Hz}$

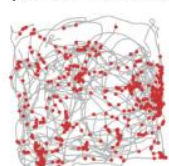

C

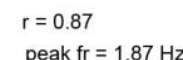

peak $\mathrm{fr}=1.87 \mathrm{~Hz}$

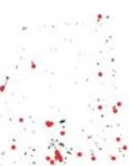

peak $\mathrm{fr}=4.50 \mathrm{~Hz}$

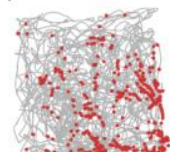

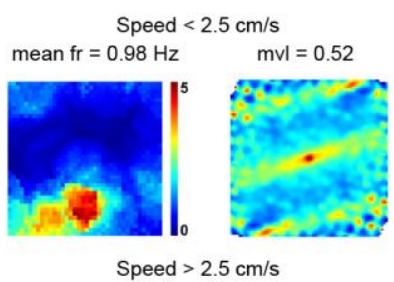
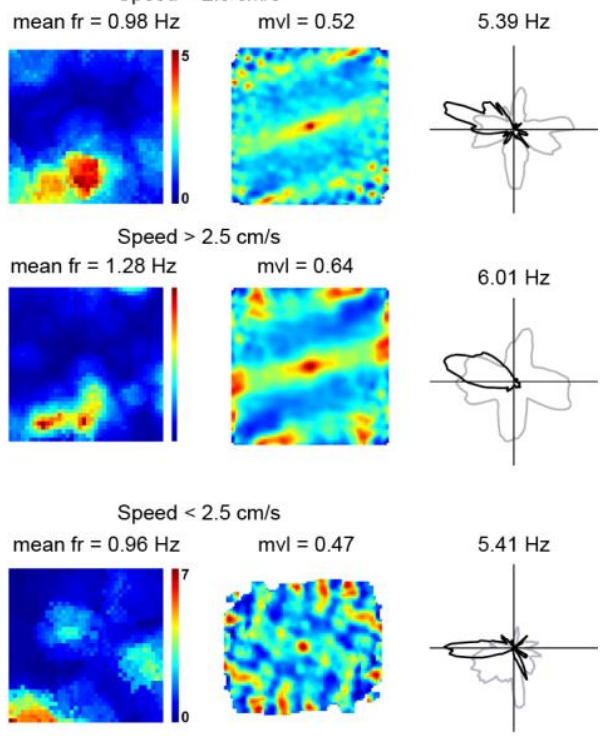

Speed $>2.5 \mathrm{~cm} / \mathrm{s}$
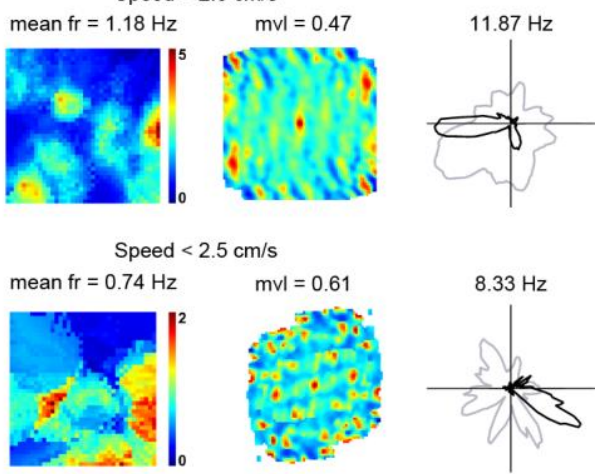

Speed $>2.5 \mathrm{~cm} / \mathrm{s}$

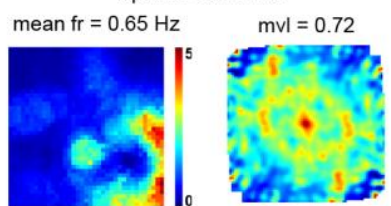

Fig. S21 Comparison of the spatial response of somatosensory head direction cells between active running and slow immobility.

(a-c) Comparison of the spatial response of representative head direction cells from Fig.

2a with instantaneous running speeds $<2.5 \mathrm{~cm} / \mathrm{s}$ and instantaneous running speeds $>$ $2.5 \mathrm{~cm} / \mathrm{s}$, respectively. Trajectory (grey line) with superimposed spike locations (red dots) (left column); rate maps (middle left column), autocorrelation maps (middle right column) and head direction tuning curves (black) plotted against dwell-time polar plot (grey) (right column) for instantaneous running speeds $<2.5 \mathrm{~cm} / \mathrm{s}$ and instantaneous running speeds $>2.5 \mathrm{~cm} / \mathrm{s}$, respectively. Firing rate is color-coded with blue indicating minimum firing rate and red indicating maximum firing rate. The scale of the 
1058 autocorrelation maps is twice that of the spatial firing rate maps. Peak firing rate (fr), 1059 mean firing rate (fr) and mean vector length (mvl) are labelled at the top of the plots. 1060 Correlation coefficients of the distributed firing rate across all directional bins when the 1061 instantaneous running speeds $<2.5 \mathrm{~cm} / \mathrm{s}$ and instantaneous running speeds $>2.5 \mathrm{~cm} / \mathrm{s}$ are indicated with $r$ at the top-left corner. 
bioRxiv preprint doi: https://doi.org/10.1101/473090; this version posted October 26, 2020. The copyright holder for this preprint (which was not certified by peer review) is the author/funder, who has granted bioRxiv a license to display the preprint in perpetuity. It is made available under aCC-BY-NC-ND 4.0 International license.
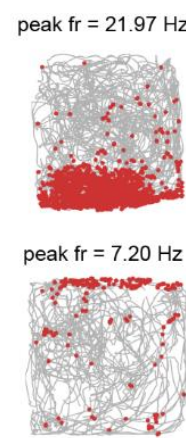

peak $\mathrm{fr}=6.69 \mathrm{~Hz}$

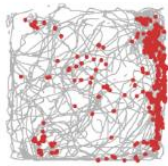

peak $\mathrm{fr}=12.62 \mathrm{~Hz}$

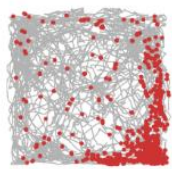

peak $\mathrm{fr}=5.31 \mathrm{~Hz}$

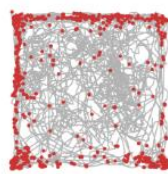

peak $\mathrm{fr}=20.33 \mathrm{~Hz}$

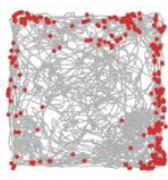

peak $\mathrm{fr}=12.80 \mathrm{~Hz}$

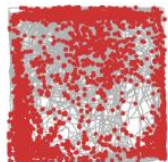

peak $\mathrm{fr}=19.74 \mathrm{~Hz}$

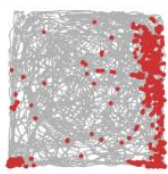

peak $\mathrm{fr}=8.08 \mathrm{~Hz}$

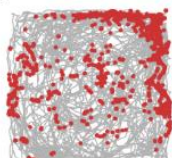

peak $\mathrm{fr}=6.63 \mathrm{~Hz}$

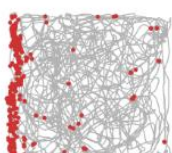

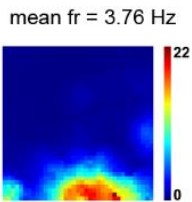

mean $\mathrm{fr}=0.40 \mathrm{~Hz}$

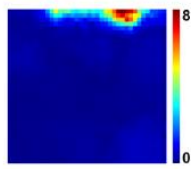

mean $\mathrm{fr}=0.42 \mathrm{~Hz}$

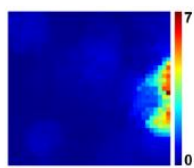

mean $\mathrm{fr}=0.63 \mathrm{~Hz}$

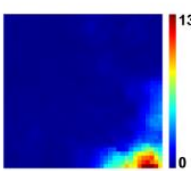

mean $\mathrm{fr}=1.01$
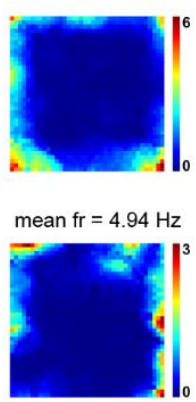

mean $\mathrm{fr}=2.88 \mathrm{~Hz}$

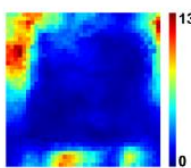

mean $\mathrm{fr}=1.09 \mathrm{~Hz}$

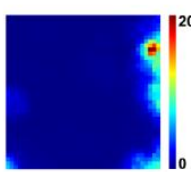

mean $\mathrm{fr}=0.72 \mathrm{~Hz}$

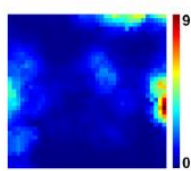

mean $\mathrm{fr}=0.40 \mathrm{~Hz}$

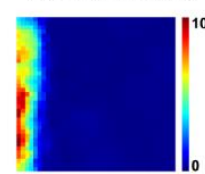

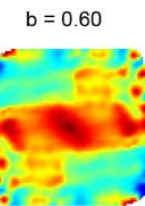
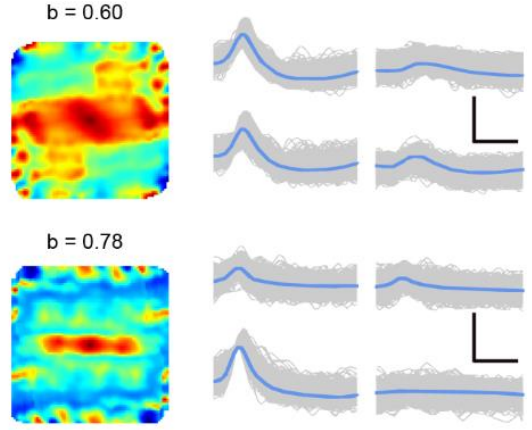

$b=0.67$
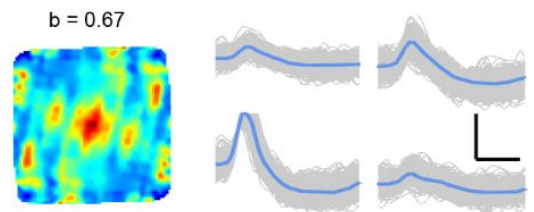

$b=0.59$

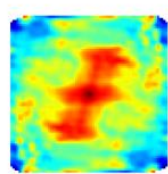

$b=0.79$
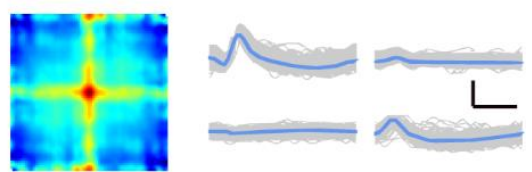

$b=0.71$
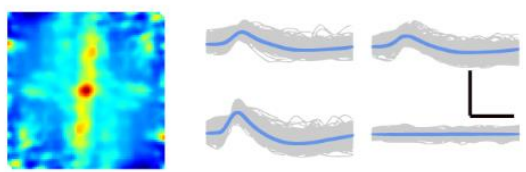

$b=0.63$
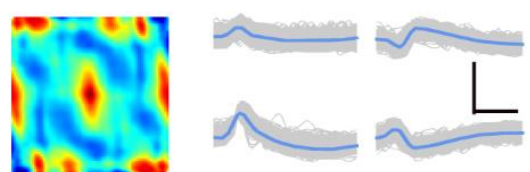

$b=0.71$
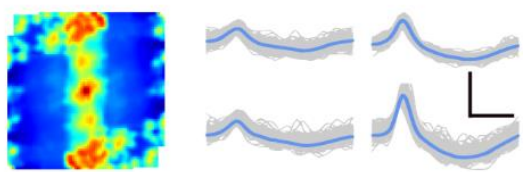

$b=0.61$
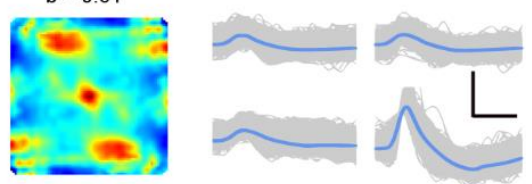

$b=0.80$
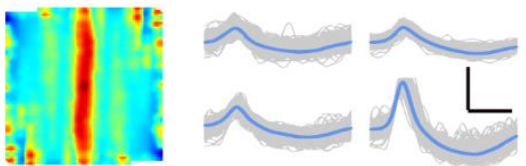
(a-c) Representative somatosensory border cells with bin coverage over $90 \%$.

1067 Trajectory (grey line) with superimposed spike locations (red dots) (left column);

1068 spatial firing rate maps (middle left column), autocorrelation diagrams (middle right 1069 column) and head direction tuning curves (black) plotted against dwell-time polar plot 1070 (grey) (right column). Firing rate is color-coded with dark blue indicating minimal 1071 firing rate and dark red indicating maximal firing rate. The scale of the autocorrelation 1072 maps is twice that of the spatial firing rate maps. Peak firing rate (fr), mean firing rate 1073 (fr), mean vector length (mvl) and angular peak rate for each representative head 1074 direction cell are labelled at the top of the panels. The directional plots show strong 1075 head direction tuning. Spike waveforms on four electrodes are shown on the right 1076 column. Scale bar, $150 \mu \mathrm{V}, 300 \mu \mathrm{s}$. 
a
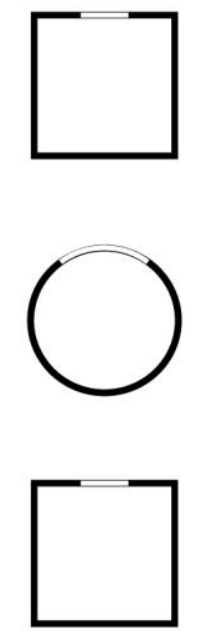

b
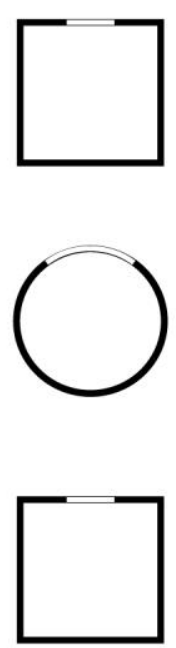

C
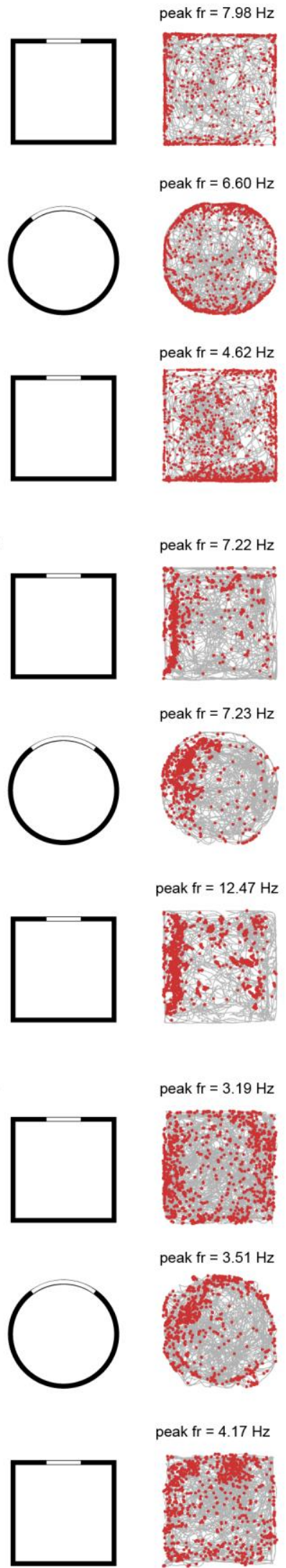

peak $\mathrm{fr}=7.23 \mathrm{~Hz}$

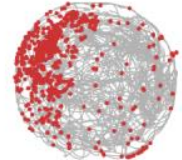

peak $\mathrm{fr}=12.47 \mathrm{~Hz}$
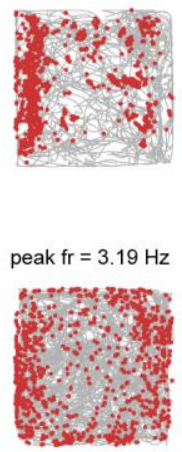

peak $\mathrm{fr}=4.17 \mathrm{~Hz}$

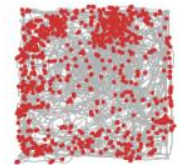

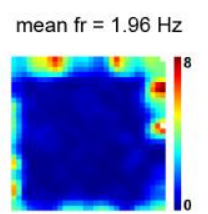
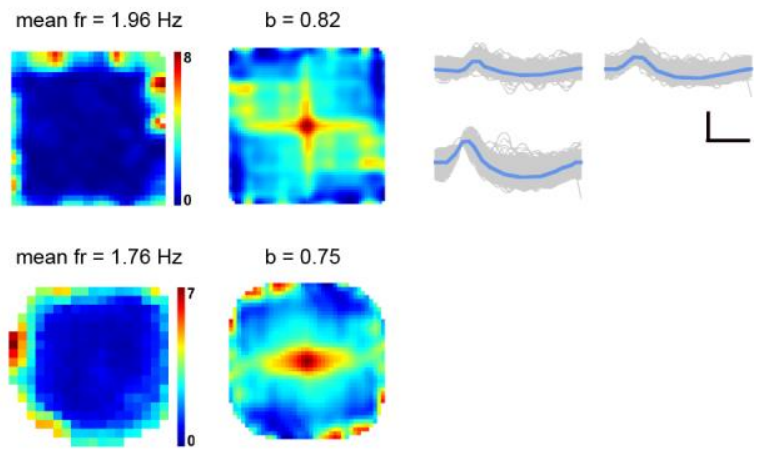

$\mathrm{b}=0.75$
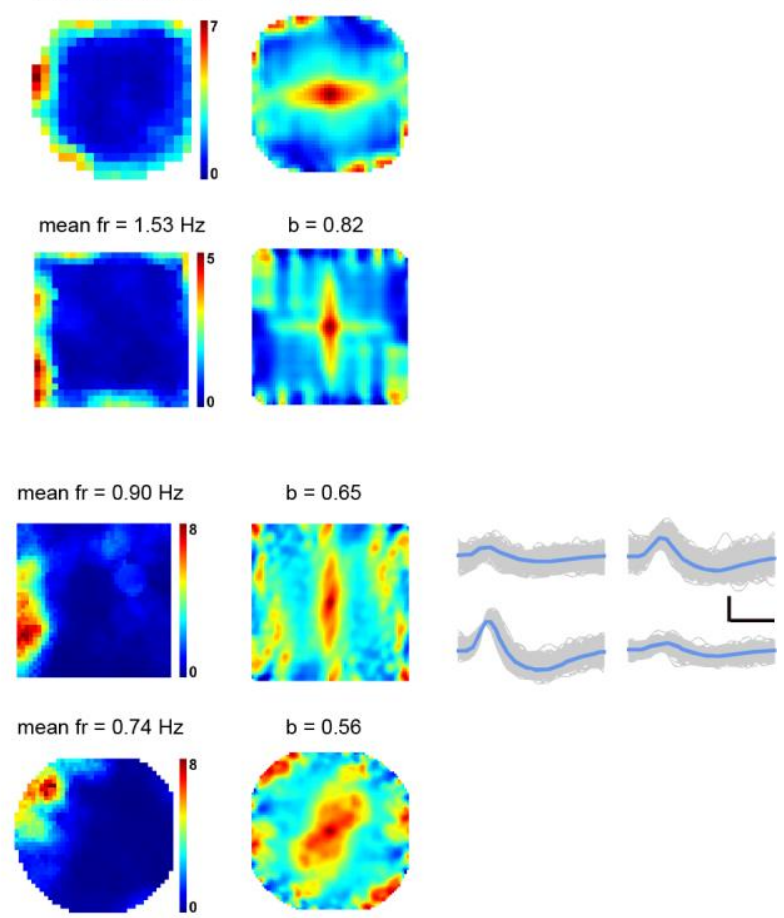

$b=0.56$

mean $\mathrm{fr}=1.68 \mathrm{~Hz}$

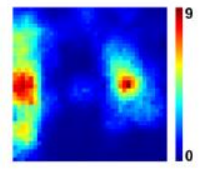

$b=0.23$
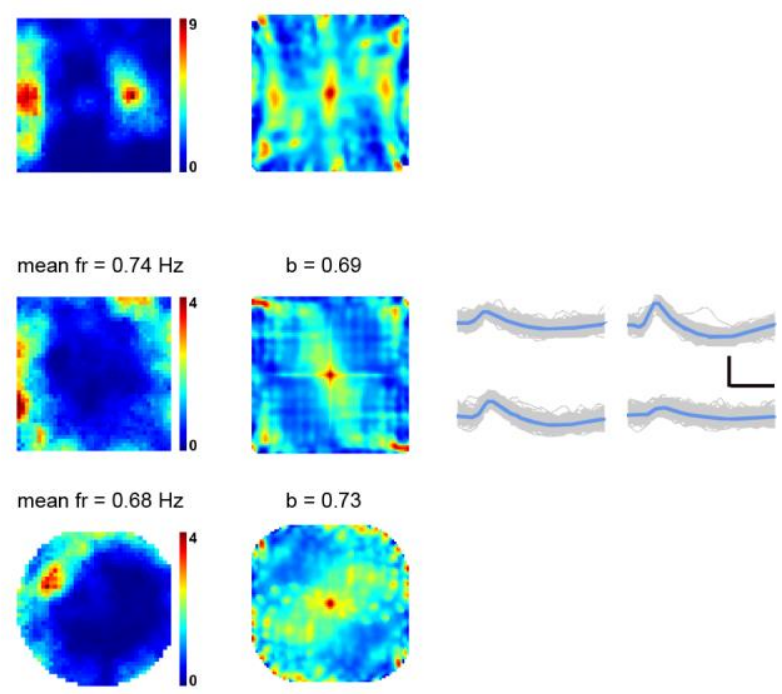

$b=0.73$
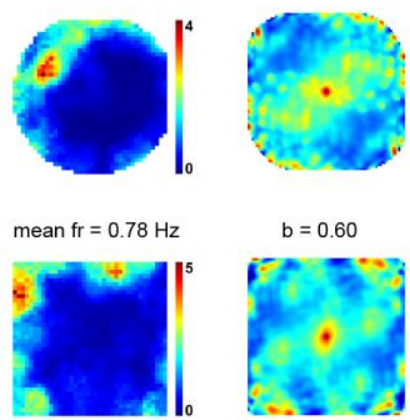

$b=0.60$

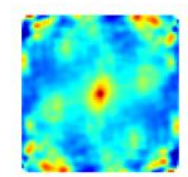

Fig. S23 Preserved firing across different geometric shapes of somatosensory border cells. 
1080 (a-c) Three representative S1 border cells preserve the firing patterns in the square and 1081 cylindrical box. Top panels, original square running box; middle panels, cylindrical box; 1082 bottom panels, back to the original square box. The experimental diagram (left column); trajectory (grey line) with superimposed spike locations (red dots) (middle left column); rate maps (middle right column) and autocorrelation diagrams (right column) for each recording trail. Firing rate is color-coded with blue indicating minimum firing rate and red indicating maximum firing rate. The scale of the autocorrelation maps is twice that 1087 of the spatial firing rate maps. Peak firing rate (fr), mean firing rate (fr) and border score 1088 (b) for each recording session are labelled at the top of the panels. Spike waveforms on four electrodes are shown on the right column. Scale bar, $200 \mu \mathrm{V}, 300 \mu \mathrm{s}$. 
a
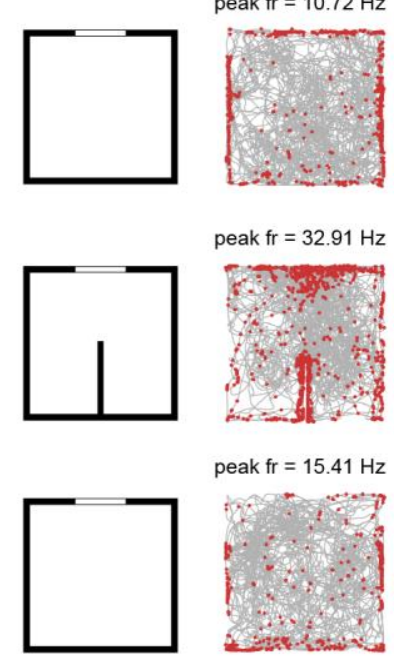

b
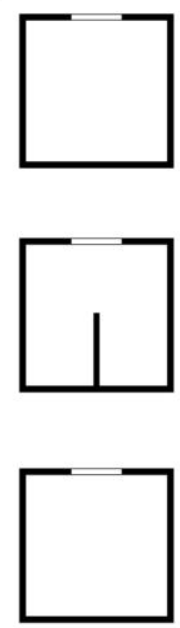

C
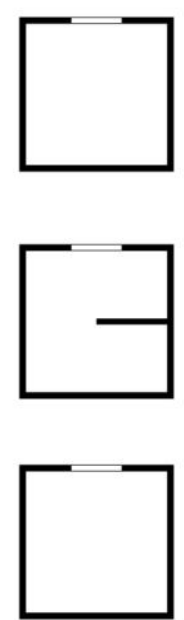

peak $\mathrm{fr}=32.91 \mathrm{~Hz}$

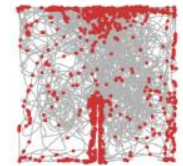

peak $\mathrm{fr}=15.41 \mathrm{~Hz}$

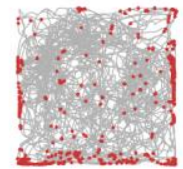

peak $\mathrm{fr}=3.82 \mathrm{~Hz}$

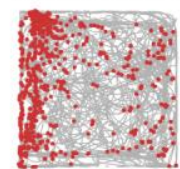

peak $\mathrm{fr}=4.21 \mathrm{~Hz}$

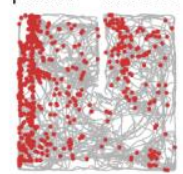

peak $\mathrm{fr}=4.93 \mathrm{~Hz}$
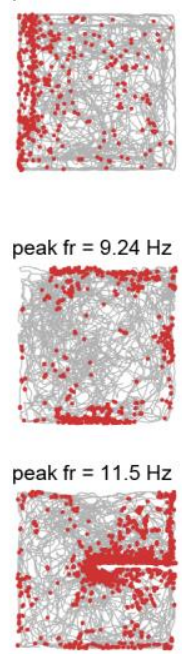

peak $\mathrm{fr}=21.01 \mathrm{~Hz}$

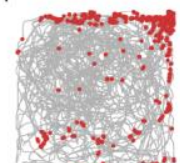

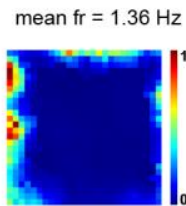

$b=0.86$
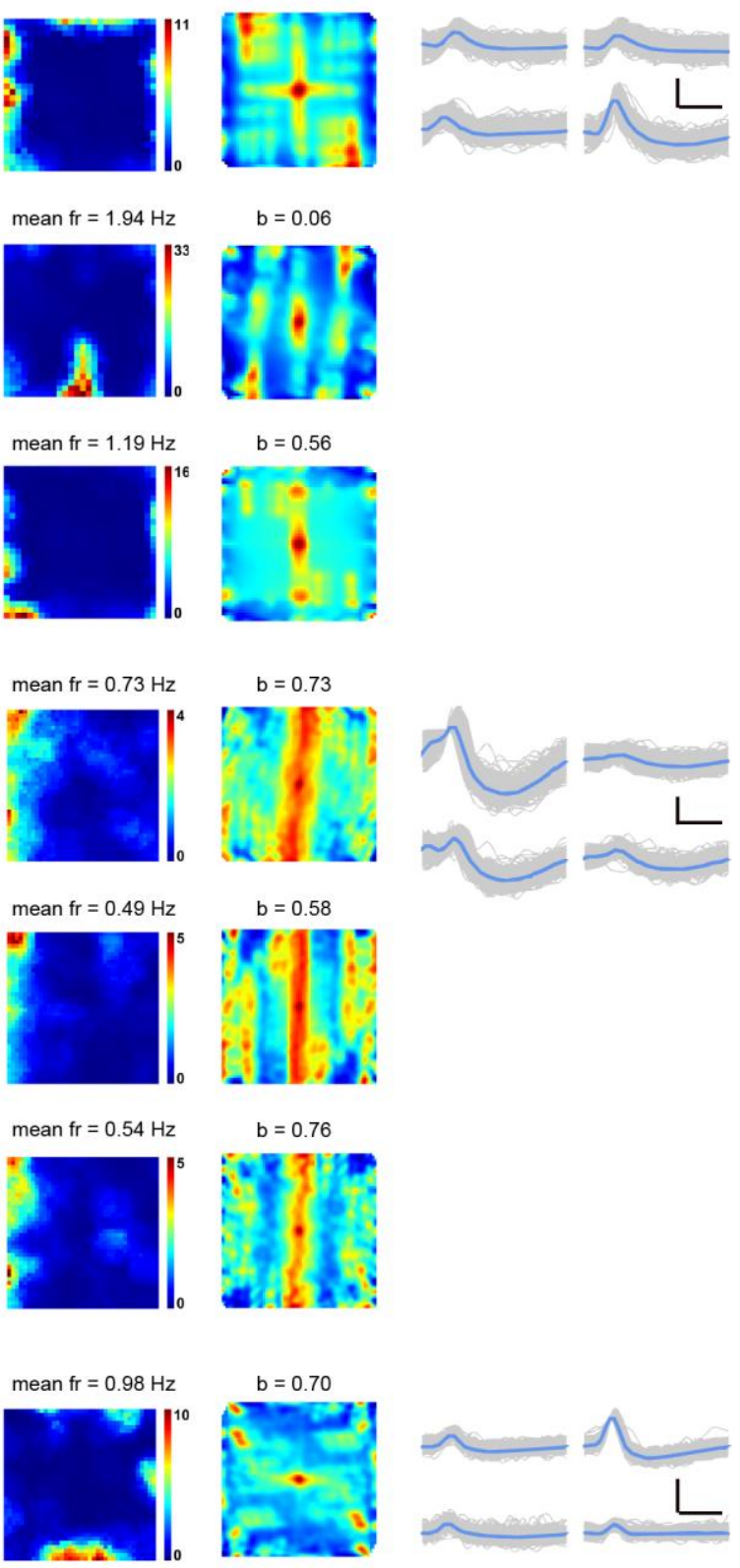

$b=0.58$

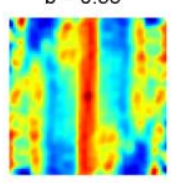

$b=0.76$

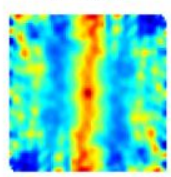

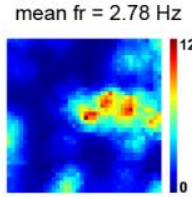

$b=-0.28$

mean $\mathrm{fr}=0.88 \mathrm{~Hz}$
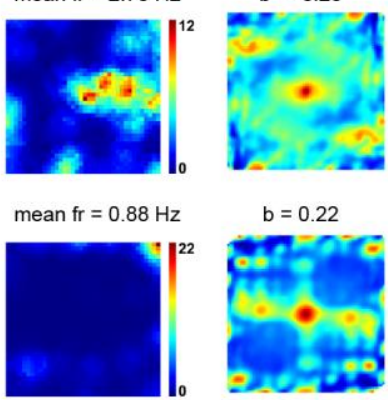

$b=0.22$

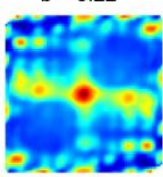

Fig. S24 Spatial responses to the external inserts of somatosensory border cells.

(a-c) Three representative somatosensory border cells recorded in the square enclosure middle panels, the same square enclosure with an internal insert; bottom panels, the 
1095 same square enclosure after removing the internal insert. The experimental diagram 1096 (left column); trajectory (grey line) with superimposed spike locations (red dots) 1097 (middle left column); rate maps (middle right column) and autocorrelation diagrams 1098 (right column) for each recording trail. Firing rate is color-coded with blue indicating 1099 minimum firing rate and red indicating maximum firing rate. The scale of the 1100 autocorrelation maps is twice that of the spatial firing rate maps. Peak firing rate (fr), 1101 mean firing rate (fr) and border score (b) for each recording session are labelled at the 1102 top of the panels. Spike waveforms on four electrodes are shown on the right column. 1103 Scale bar, $150 \mu \mathrm{V}, 300 \mu \mathrm{s}$. 
bioRxiv preprint doi: https://doi.org/10.1101/473090; this version posted October 26, 2020. The copyright holder for this preprint (which was not certified by peer review) is the author/funder, who has granted bioRxiv a license to display the preprint in perpetuity. It is made available under aCC-BY-NC-ND 4.0 International license.

a Square Enclosure
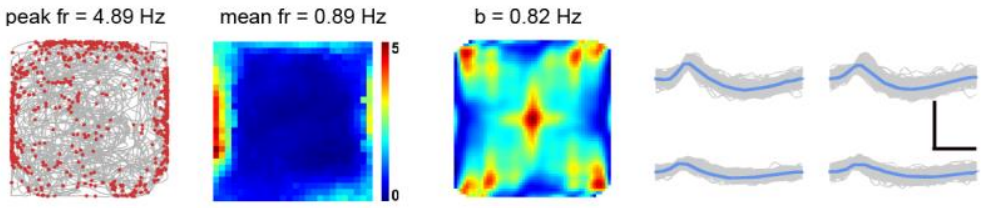

Elevated Platform without Walls
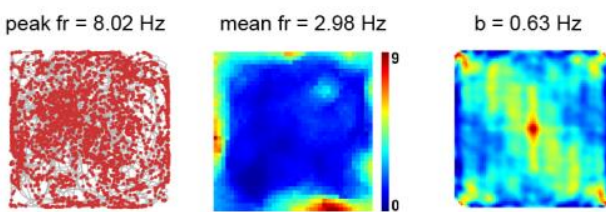

Square Enclosure

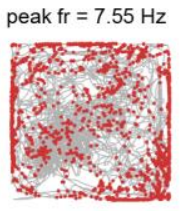

mean $\mathrm{fr}=1.57 \mathrm{~Hz}$

$b=0.84 \mathrm{~Hz}$
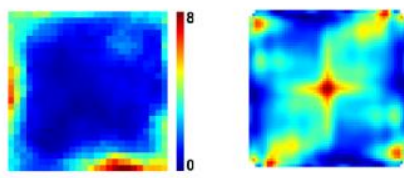

b

Square Enclosure
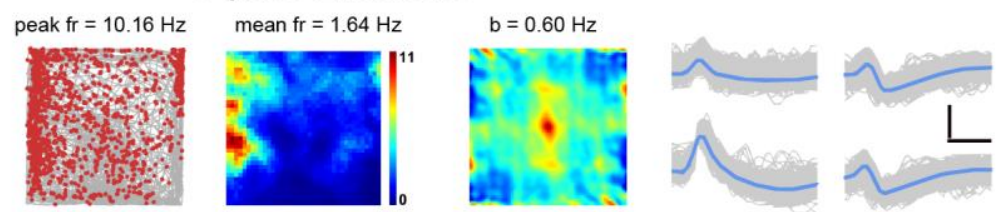

Elevated Platform without Walls
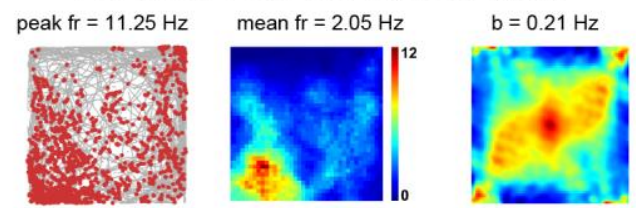

Square Enclosure
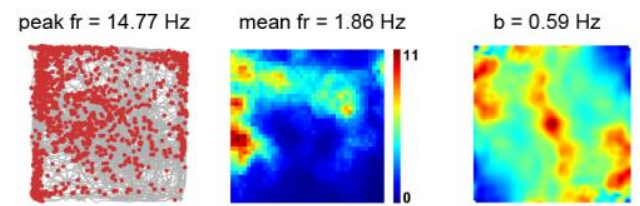

C

Square Enclosure
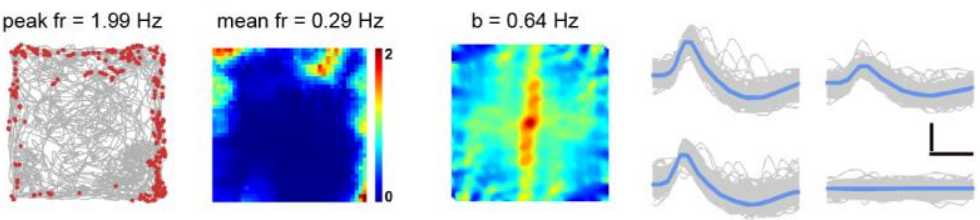

Elevated Platform without Walls
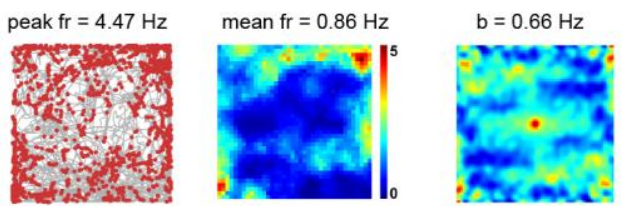

Square Enclosure
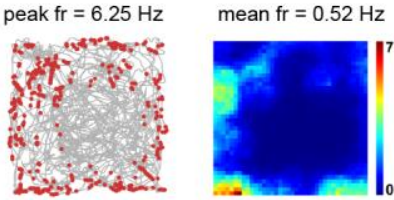

$\mathrm{b}=0.55 \mathrm{~Hz}$

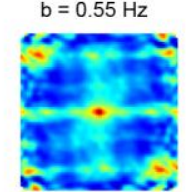


Fig. S25 Somatosensory border cells recorded from the elevated platform without walls.

(a-c) Spatial responses of three representative somatosensory border cells recorded in the square box, in the elevated platform without walls and back to the square box. Trajectory (grey line) with superimposed spike locations (red dots) (left column); heat maps of firing rate (middle column) and autocorrelation maps (right column). Firing rate is color-coded with blue indicating minimum firing rate and red indicating maximum firing rate. The scale of the autocorrelation maps is twice that of the spatial firing rate maps. Peak firing rate (fr), mean firing rate (fr) and border score (b) for each representative border cell are labelled at the top of the panels. Spike waveforms on four electrodes are shown on the right column. Scale bar, $150 \mu \mathrm{V}, 300 \mu \mathrm{s}$.
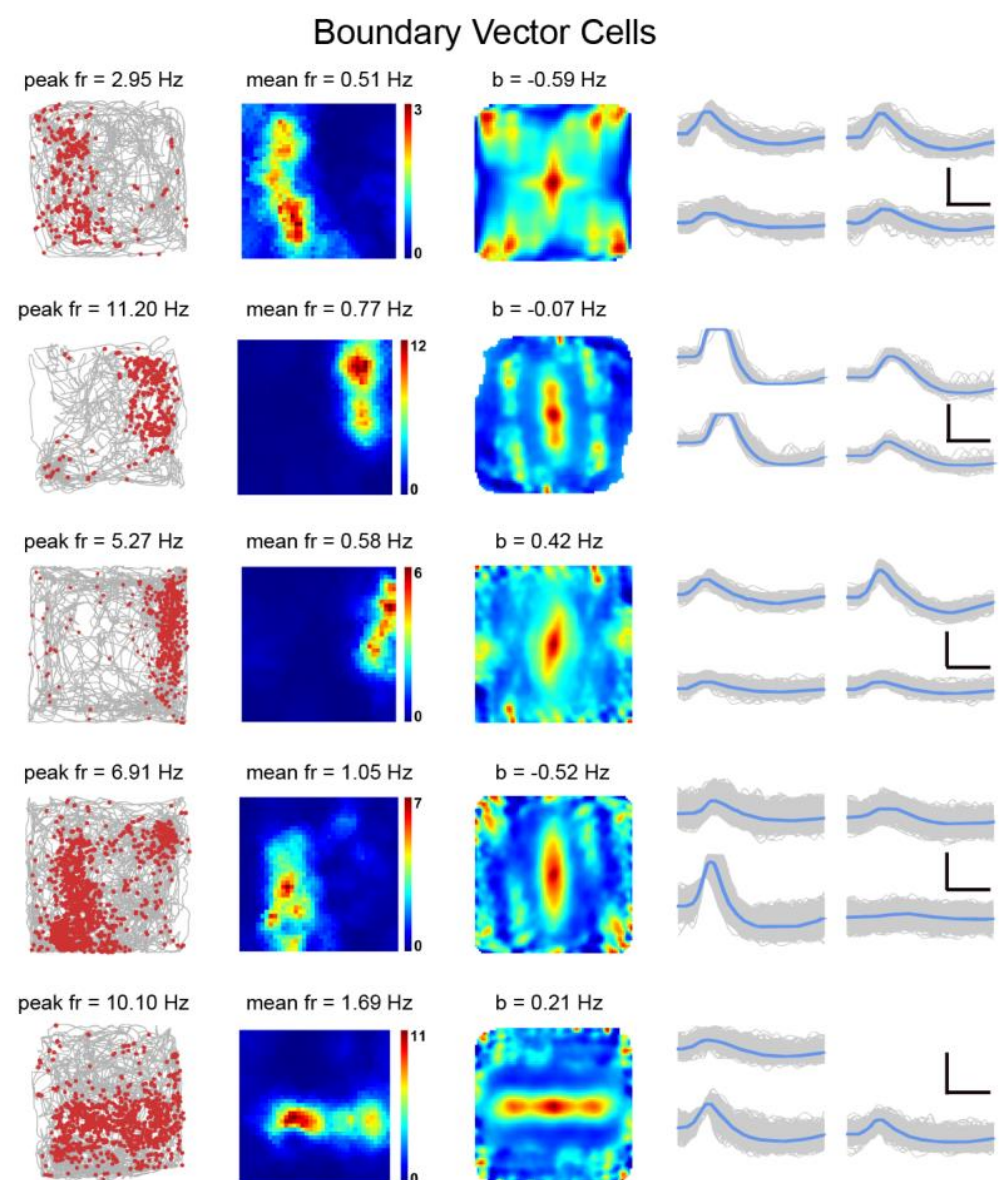

mean $\mathrm{fr}=1.69 \mathrm{~Hz}$
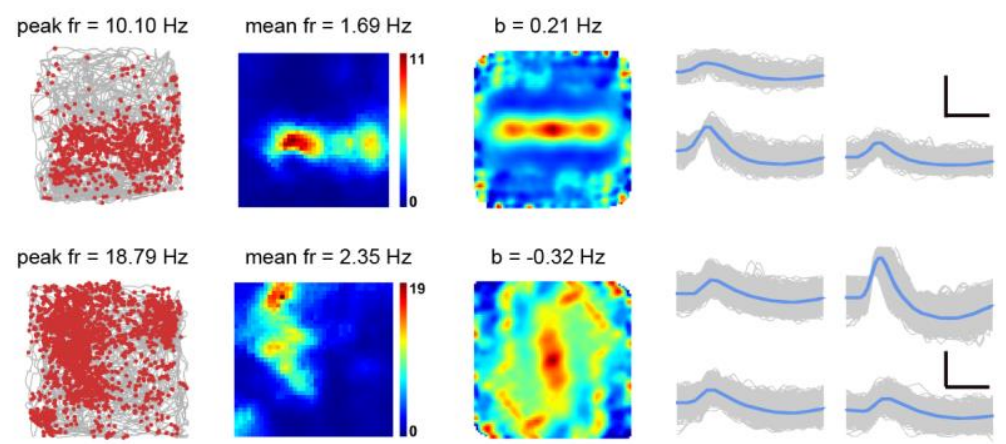
1119 Examples of somatosensory boundary vector cells. Trajectory (grey line) with 1120 superimposed spike locations (red dots) (left column); heat maps of firing rate (middle column) and autocorrelation diagrams (right column). Firing rate is color-coded with blue indicating minimum firing rate and red indicating maximum firing rate. The scale of the autocorrelation maps is twice that of the spatial firing rate maps. Peak firing rate (fr), mean firing rate (fr) and border score (b) for each representative boundary vector cell are labelled at the top of the panels. Spike waveforms on four electrodes are shown on the right column. Scale bar, $150 \mu \mathrm{V}, 300 \mu \mathrm{s}$.

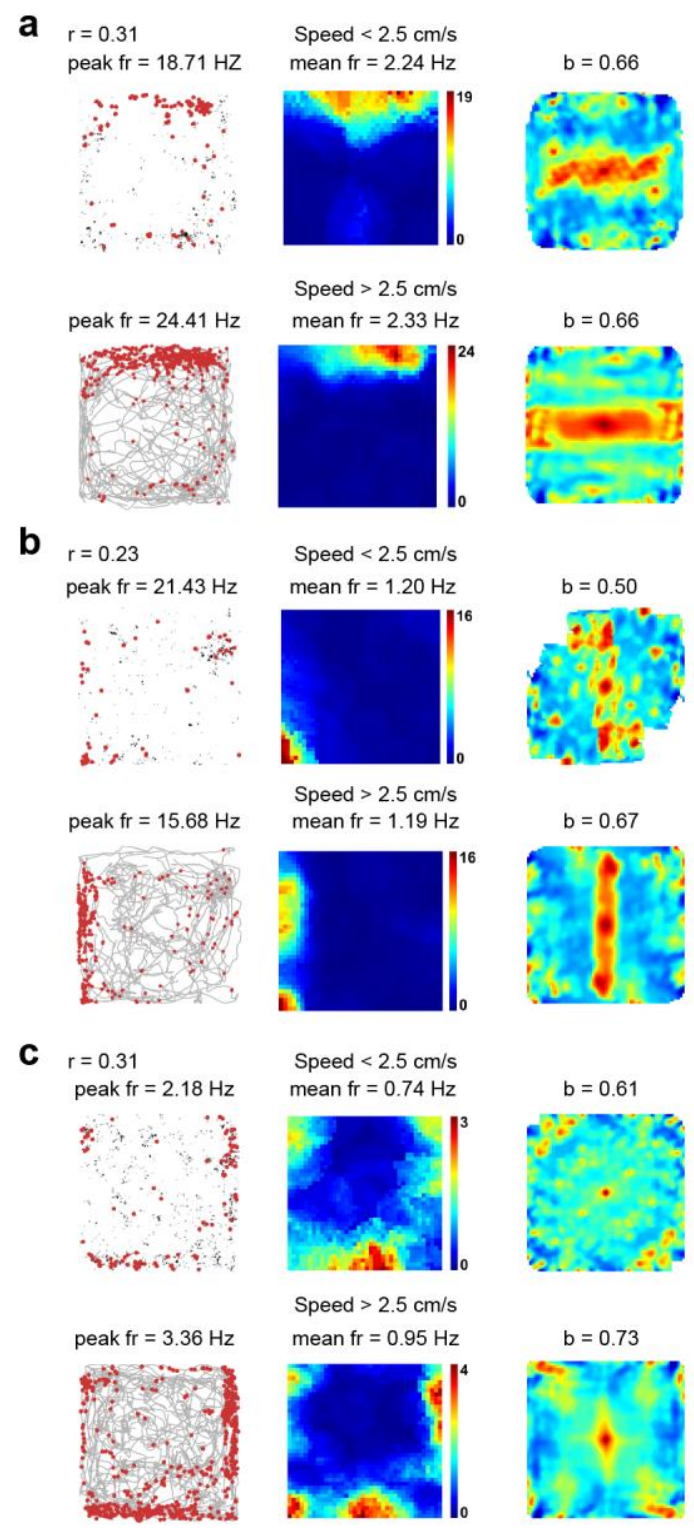




\section{9 active running and slow immobility.}

1130 (a-c) Comparison of the spatial response of representative border cells from Fig. 3a

1131 with instantaneous running speeds $<2.5 \mathrm{~cm} / \mathrm{s}$ and instantaneous running speeds $>2.5$

$1132 \mathrm{~cm} / \mathrm{s}$, respectively. Trajectory (grey line) with superimposed spike locations (red dots)

1133 (left column); rate maps (middle column) and autocorrelation maps (right column) for

1134 instantaneous running speeds $<2.5 \mathrm{~cm} / \mathrm{s}$ and instantaneous running speeds $>2.5 \mathrm{~cm} / \mathrm{s}$,

1135 respectively. Firing rate is color-coded with blue indicating minimum firing rate and

1136 red indicating maximum firing rate. The scale of the autocorrelation maps is twice that

1137 of the spatial firing rate maps. Peak firing rate (fr), mean firing rate (fr) and border score

1138 (b) are labelled at the top of the plots. Pearson's correlation coefficients between two

1139 firing rate maps when the instantaneous running speeds $<2.5 \mathrm{~cm} / \mathrm{s}$ and instantaneous running speeds $>2.5 \mathrm{~cm} / \mathrm{s}$ are indicated with $r$ at the top-left corner. 
bioRxiv preprint doi: https://doi.org/10.1101/473090; this version posted October 26, 2020. The copyright holder for this preprint (which was not certified by peer review) is the author/funder, who has granted bioRxiv a license to display the preprint in perpetuity. It is made available under aCC-BY-NC-ND 4.0 International license.
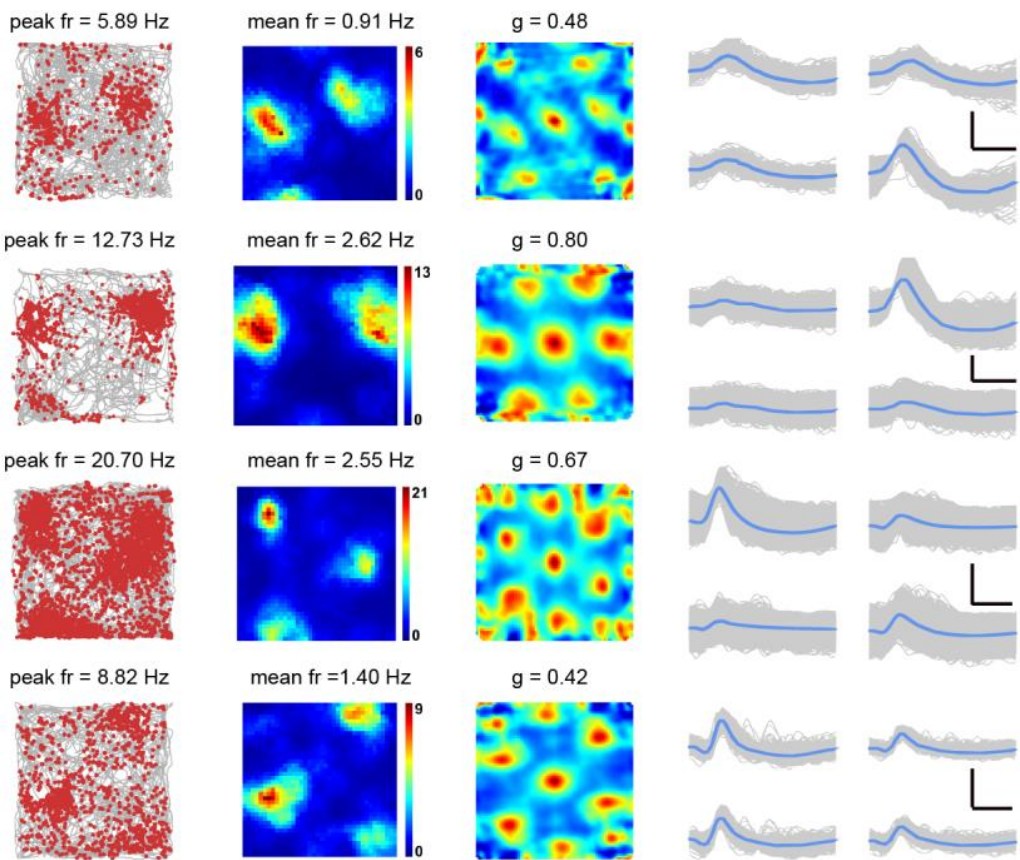

mean $\mathrm{fr}=2.62 \mathrm{~Hz}$

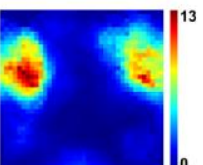

$g=0.80$
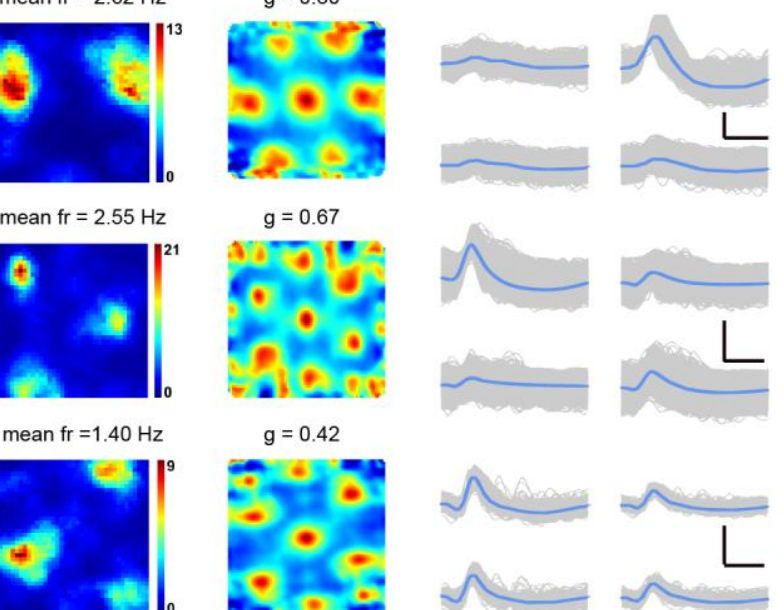

$\mathrm{g}=0.42$
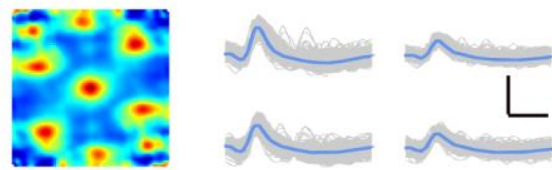

peak $\mathrm{fr}=6.15 \mathrm{~Hz}$
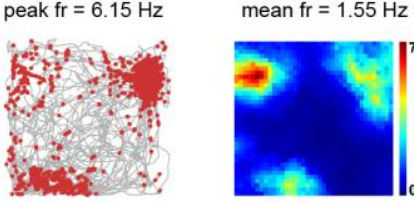

peak $\mathrm{fr}=5.68 \mathrm{~Hz}$

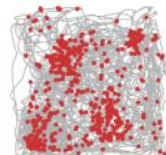

mean $\mathrm{fr}=0.59 \mathrm{~Hz}$
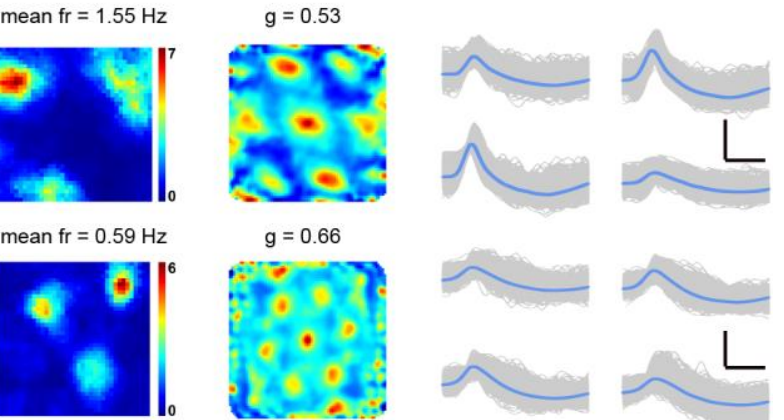

peak $\mathrm{fr}=19.11 \mathrm{~Hz}$

mean $\mathrm{fr}=3.72 \mathrm{~Hz}$
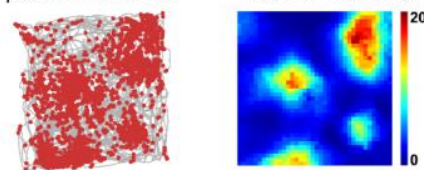

$g=0.66$
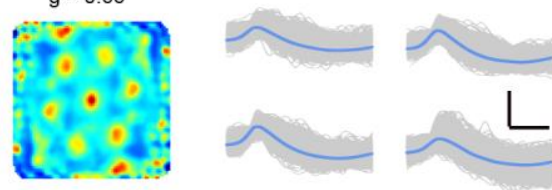

peak $\mathrm{fr}=13.36 \mathrm{~Hz}$

mean $\mathrm{fr}=0.98 \mathrm{~Hz}$
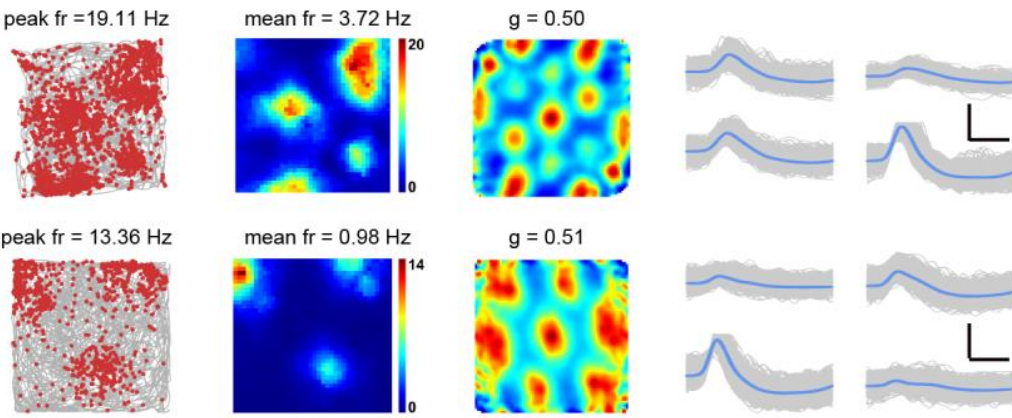

$\mathrm{g}=0.51$
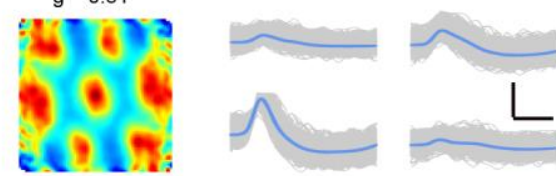

peak $\mathrm{fr}=7.52 \mathrm{~Hz}$

mean $\mathrm{fr}=1.15 \mathrm{~Hz}$
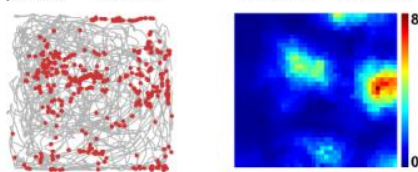

$\mathrm{g}=0.76$
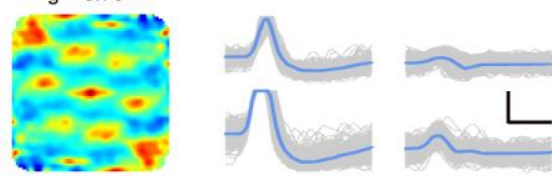

peak $\mathrm{fr}=5.02 \mathrm{~Hz}$

mean $\mathrm{fr}=0.95 \mathrm{~Hz}$
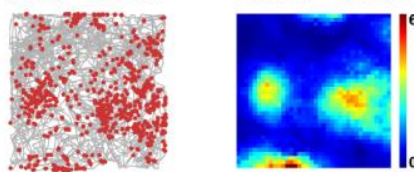

$g=0.56$
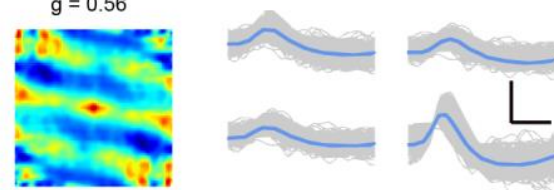

1142 Fig. S28 More examples of somatosensory grid cells recorded from the 
1144 Representative somatosensory grid cells with bin coverage over 90\%. Trajectory (grey

1145 line) with superimposed spike locations (red dots) (left column); spatial firing rate maps

1146 (middle column) and autocorrelation diagrams (right column). Firing rate is color-

1147 coded with dark blue indicating minimal firing rate and dark red indicating maximal

1148 firing rate. The scale of the autocorrelation maps is twice that of the spatial firing rate

1149 maps. Peak firing rate (fr), mean firing rate (fr) and grid score (g) for each representative

1150 head direction cell are labelled at the top of the panels. The directional plots show strong

1151 head direction tuning. Spike waveforms on four electrodes are shown on the right

1152 column. Scale bar, $150 \mu \mathrm{V}, 300 \mu \mathrm{s}$. 
a
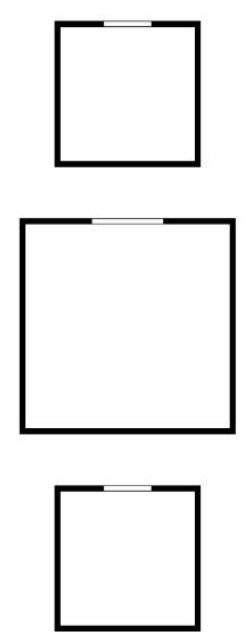

b
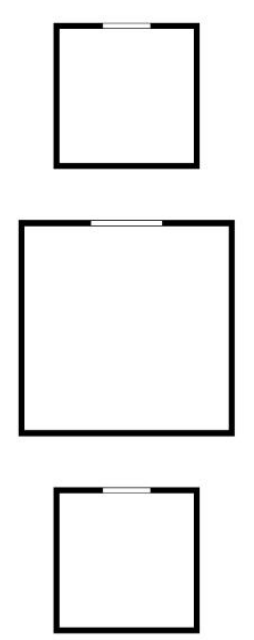

C
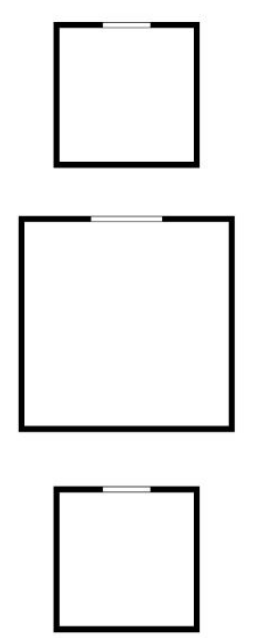

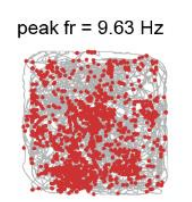

peak $\mathrm{fr}=14.18 \mathrm{~Hz}$

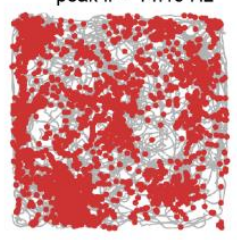

peak fr $=11.65 \mathrm{~Hz}$
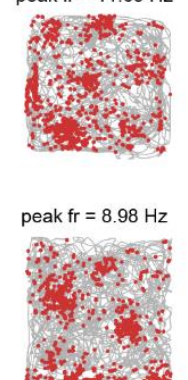

peak $\mathrm{fr}=22.56 \mathrm{~Hz}$

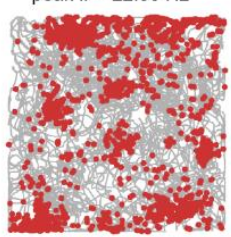

peak $\mathrm{fr}=18.88 \mathrm{~Hz}$
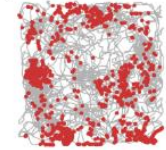

peak fr $=1.51 \mathrm{~Hz}$

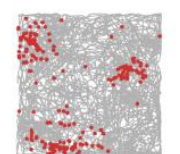

peak $\mathrm{fr}=1.98 \mathrm{~Hz}$

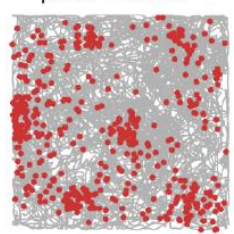

peak $\mathrm{fr}=1.00 \mathrm{~Hz}$

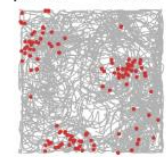

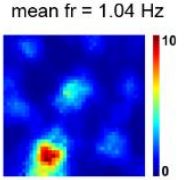
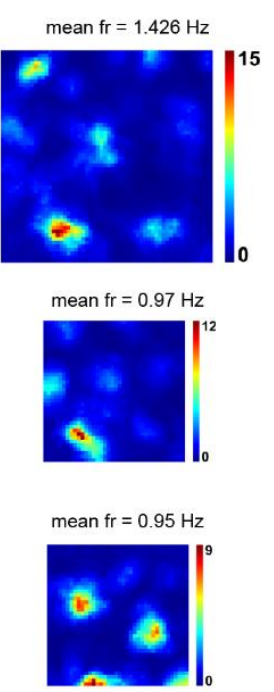

mean $\mathrm{fr}=1.53 \mathrm{~Hz}$

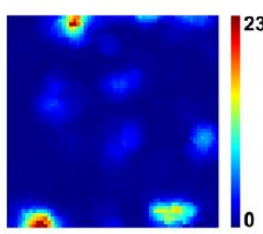

mean $\mathrm{fr}=1.05 \mathrm{~Hz}$
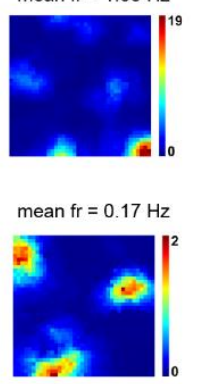

mean $\mathrm{fr}=0.20 \mathrm{~Hz}$
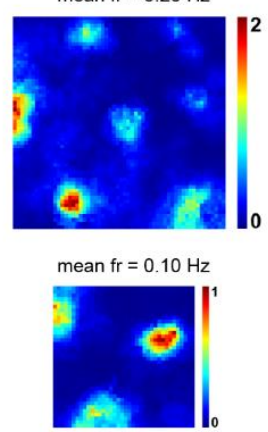

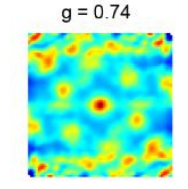

$g=0.55$
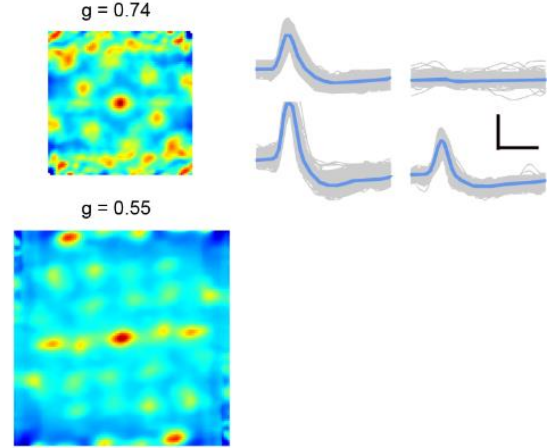

$\mathrm{g}=0.72$

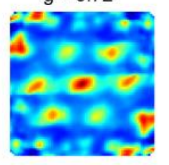

$g=0.81$
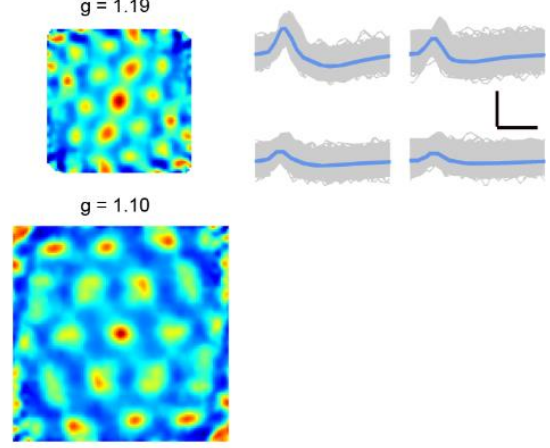

$g=1.34$
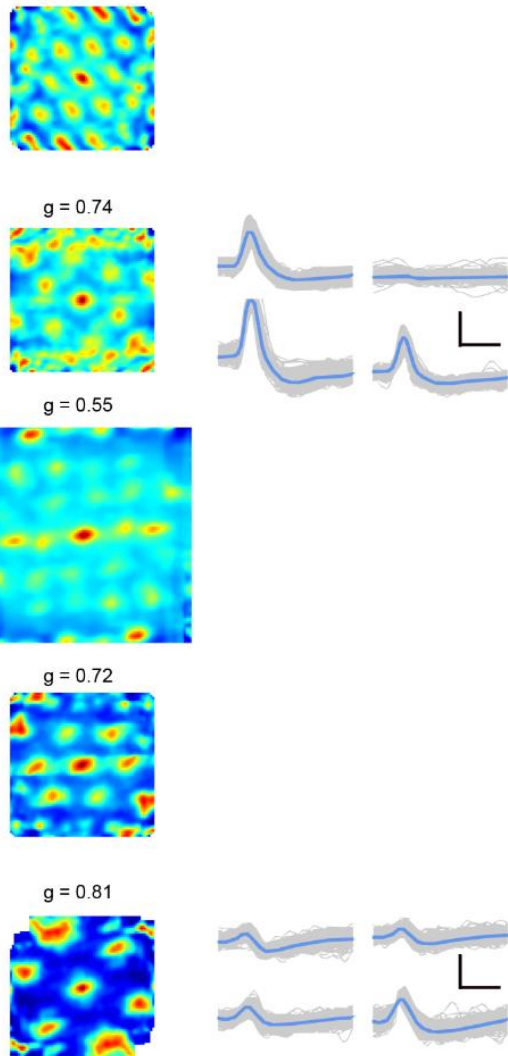
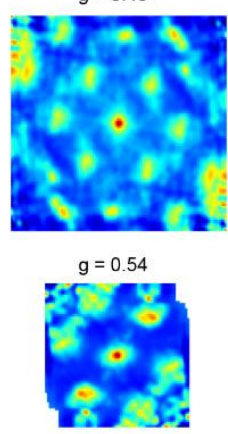

Fig. S29 Somatosensory grid cells recorded in the larger environment.

(a-c) Three examples of somatosensory grid cells recorded in $1 \mathrm{~m} \times 1 \mathrm{~m}, 1.5 \mathrm{~m} \times 1.5 \mathrm{~m}$ and $1 \mathrm{~m} \times 1 \mathrm{~m}$ square box. The experimental diagram (left column); trajectory (grey line) with superimposed spike locations (red dots) (middle left column); spatial firing rate 
maps (middle right column) and autocorrelation diagrams (right column). Firing rate is color-coded with dark blue indicating minimal firing rate and dark red indicating maximal firing rate. The scale of the autocorrelation maps is twice that of the spatial firing rate maps. Peak firing rate (fr), mean firing rate (fr) and grid score $(\mathrm{g})$ for each representative head direction cell are labelled at the top of the panels. Spike waveforms on four electrodes are shown on the right column. Scale bar, $150 \mu \mathrm{V}, 300 \mu \mathrm{s}$.

a

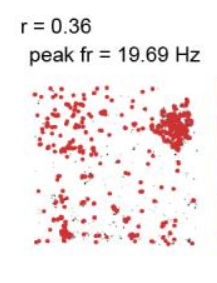

Speed $<2.5 \mathrm{~cm} / \mathrm{s}$

mean $\mathrm{fr}=2.98 \mathrm{~Hz}$
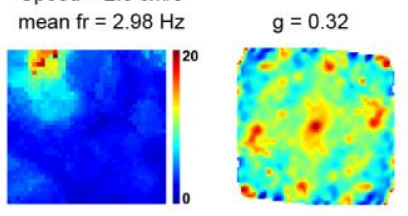

peak $\mathrm{fr}=18.00 \mathrm{~Hz}$

Speed $>2.5 \mathrm{~cm} / \mathrm{s}$
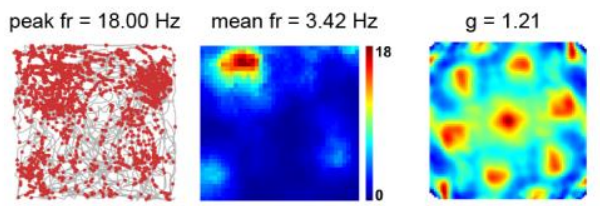

b

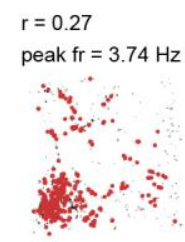

Speed $<2.5 \mathrm{~cm} / \mathrm{s}$

mean $\mathrm{fr}=0.66 \mathrm{~Hz}$
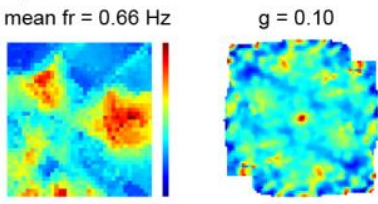

peak $\mathrm{fr}=16.32 \mathrm{~Hz}$

Speed $>2.5 \mathrm{~cm} / \mathrm{s}$
mean $\mathrm{fr}=2.64 \mathrm{~Hz}$
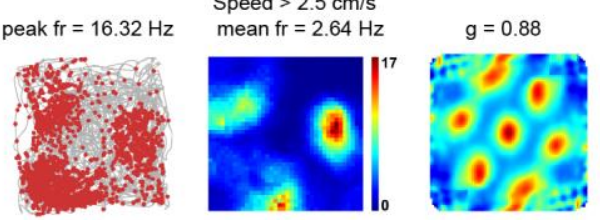

C

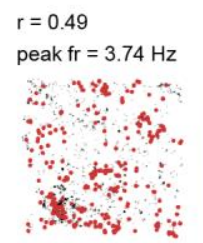

Speed $<2.5 \mathrm{~cm} / \mathrm{s}$

mean $\mathrm{fr}=0.66 \mathrm{~Hz}$
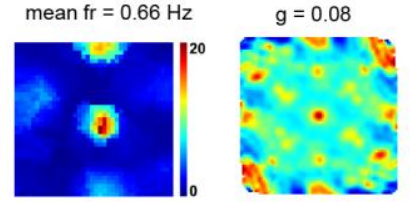

Speed $>2.5 \mathrm{~cm} / \mathrm{s}$

peak $\mathrm{fr}=16.88 \mathrm{~Hz}$

mean $\mathrm{fr}=1.87 \mathrm{~Hz}$
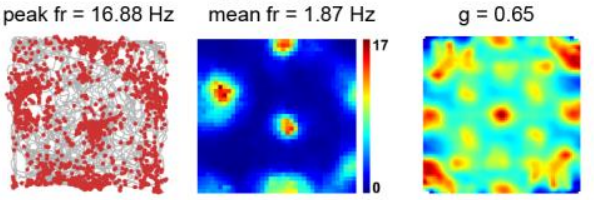

Fig. S30 Comparison of the spatial response of somatosensory grid cells between active running and slow immobility.

(a-c) Comparison of the spatial response of representative grid cells from Fig. $4 \mathbf{a}$ with instantaneous running speeds $<2.5 \mathrm{~cm} / \mathrm{s}$ and instantaneous running speeds $>2.5 \mathrm{~cm} / \mathrm{s}$, 
1169 respectively. Trajectory (grey line) with superimposed spike locations (red dots) (left

1170 column); rate maps (middle column) and autocorrelation maps (right column) for

1171 instantaneous running speeds $<2.5 \mathrm{~cm} / \mathrm{s}$ and instantaneous running speeds $>2.5 \mathrm{~cm} / \mathrm{s}$,

1172 respectively. Firing rate is color-coded with blue indicating minimum firing rate and

1173 red indicating maximum firing rate. The scale of the autocorrelation maps is twice that

1174 of the spatial firing rate maps. Peak firing rate (fr), mean firing rate (fr) and grid score

1175 (b) are labelled at the top of the plots. Pearson's correlation coefficients between two

1176 firing rate maps when the instantaneous running speeds $<2.5 \mathrm{~cm} / \mathrm{s}$ and instantaneous

1177 running speeds $>2.5 \mathrm{~cm} / \mathrm{s}$ are indicated with $r$ at the top-left corner. 


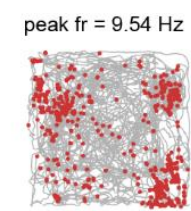

peak $\mathrm{fr}=8.50 \mathrm{~Hz}$

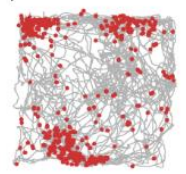

peak $\mathrm{fr}=1.68 \mathrm{~Hz}$

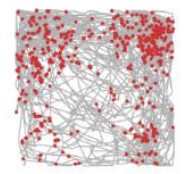

peak $\mathrm{fr}=9.29 \mathrm{~Hz}$
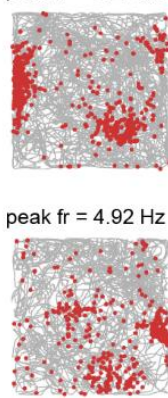

peak $\mathrm{fr}=7.12 \mathrm{~Hz}$

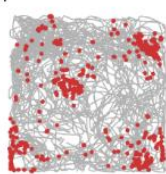

peak $\mathrm{fr}=7.12 \mathrm{~Hz}$

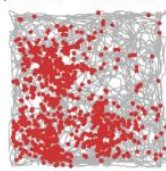

peak $\mathrm{fr}=14.36 \mathrm{~Hz}$

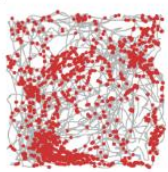

peak $\mathrm{fr}=9.81 \mathrm{~Hz}$

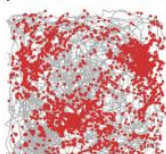

peak $\mathrm{fr}=11.30 \mathrm{~Hz}$

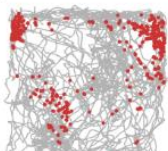

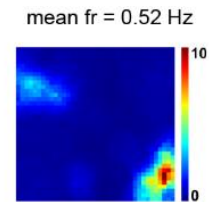

mean $\mathrm{fr}=0.55 \mathrm{~Hz}$

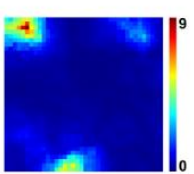

mean $\mathrm{fr}=0.55 \mathrm{~Hz}$
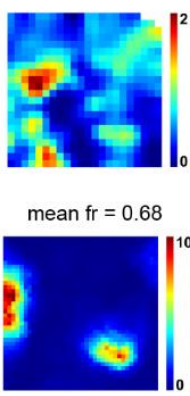

mean $\mathrm{fr}=0.43$
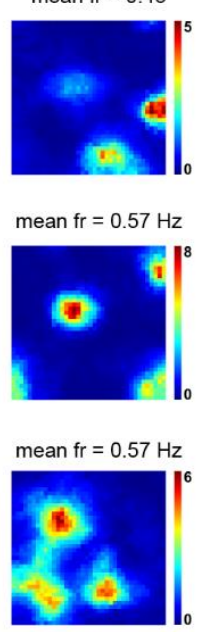

mean $\mathrm{fr}=1.54 \mathrm{~Hz}$
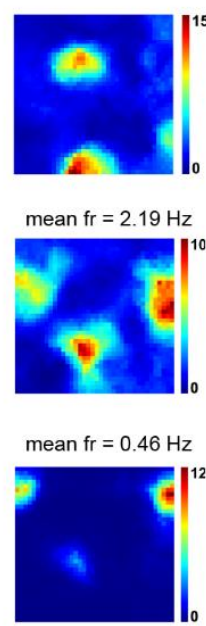
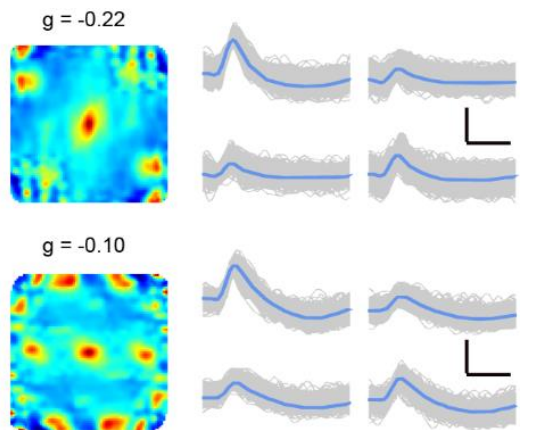

$g=-0.34$
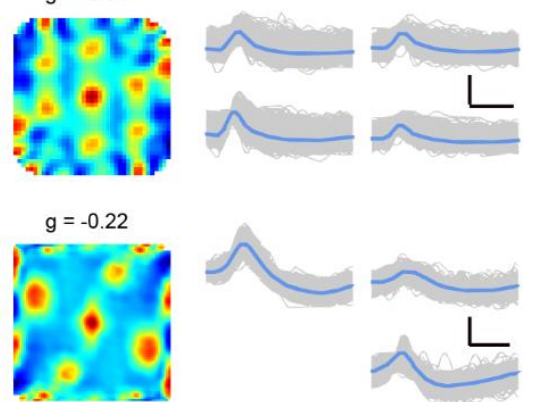

$g=0.21$
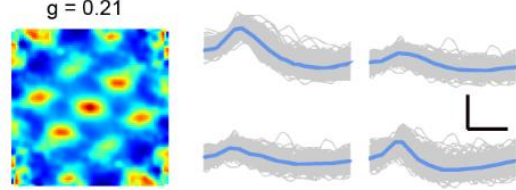

$g=-0.12$
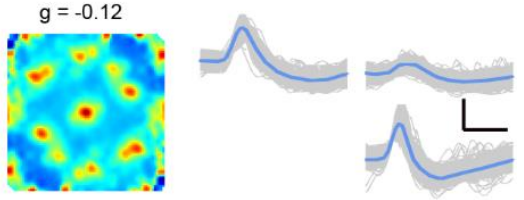

$g=-0.12$
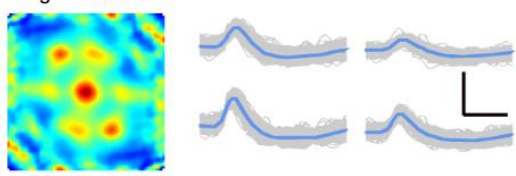

$g=-0.12$
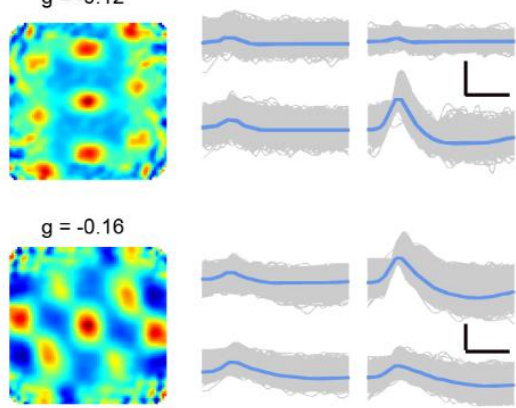

$g=-0.03$
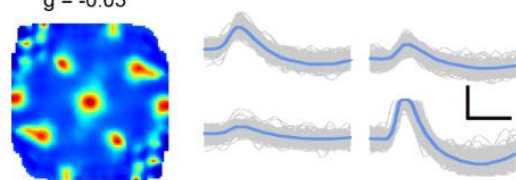
Representative somatosensory irregular grid cells with bin coverage over $90 \%$. Trajectory (grey line) with superimposed spike locations (red dots) (left column); spatial firing rate maps (middle column) and autocorrelation diagrams (right column). Firing rate is color-coded with dark blue indicating minimal firing rate and dark red indicating maximal firing rate. The scale of the autocorrelation maps is twice that of the spatial firing rate maps. Peak firing rate (fr), mean firing rate (fr) and grid score (g) for each representative head direction cell are labelled at the top of the panels. The directional plots show strong head direction tuning. Spike waveforms on four electrodes are shown on the right column. Scale bar, $150 \mu \mathrm{V}, 300 \mu \mathrm{s}$.

a
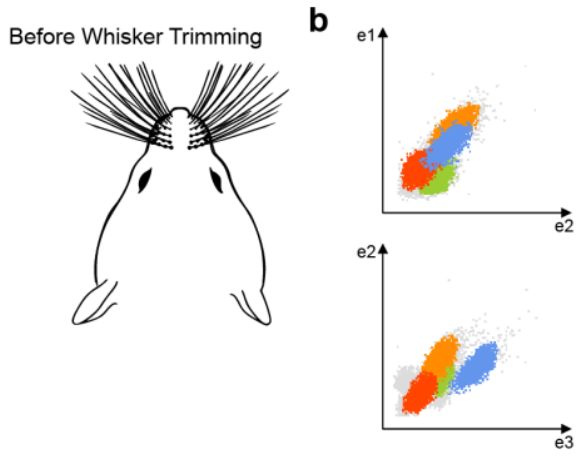

C

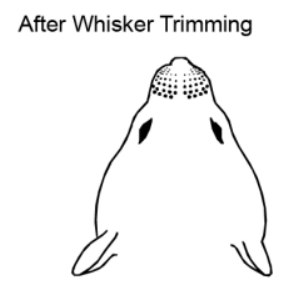

d

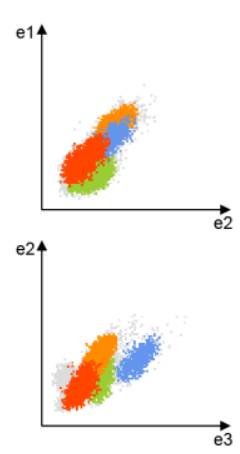

e

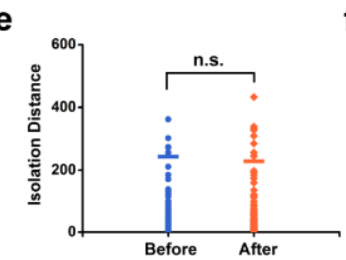

f

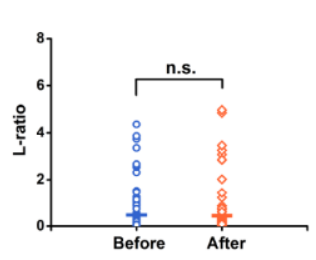

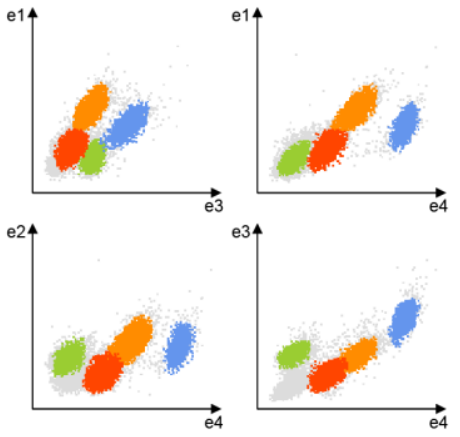
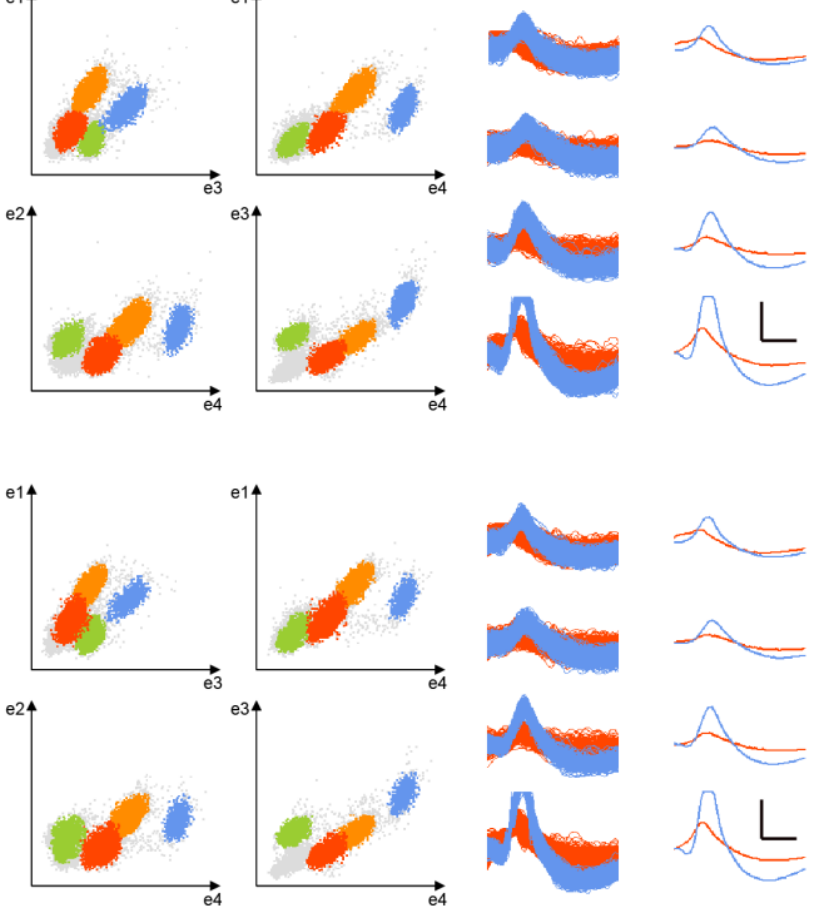

g

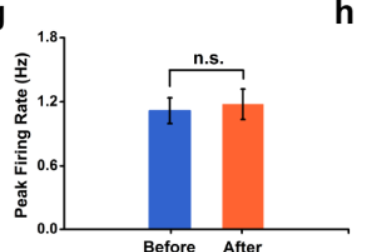

h

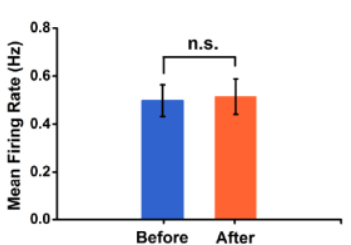

Fig. S32 Preserved firing properties of the isolated somatosensory units before and after whisker trimming.

1193 (a and $\mathbf{c}$ ) The diagram showing the rat before (a) and after (c) whisker trimming. (b and d) Scatterplots show preserved cluster diagrams before (b) and after (d) whisker trimming of the same tetrode from the same recording rat. Each individual dot 
represents a single recorded spike. Waveforms from two separated blue and red clusters in the scatterplots are shown for four electrodes from the same tetrode. The waveforms remain unchanged after whisker trimming. Scale bar, $150 \mu \mathrm{V}, 300 \mu \mathrm{s}$. (e) The comparison of isolation distance for identified S1 units right before and after whisker trimming. (f) Same as e for the L-ratio. (g) Same as in (f) for the mean firing rate. (h) Same as in (g) for the peak firing rate. $n=78$, two-tailed paired $t$-test, n.s., not significant.

a Before Whisker Trimming
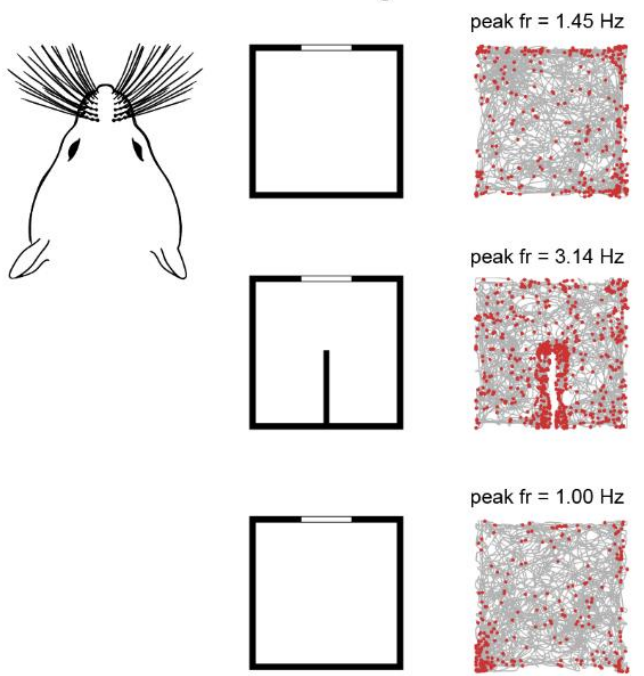

\section{b}

After Whisker Trimming
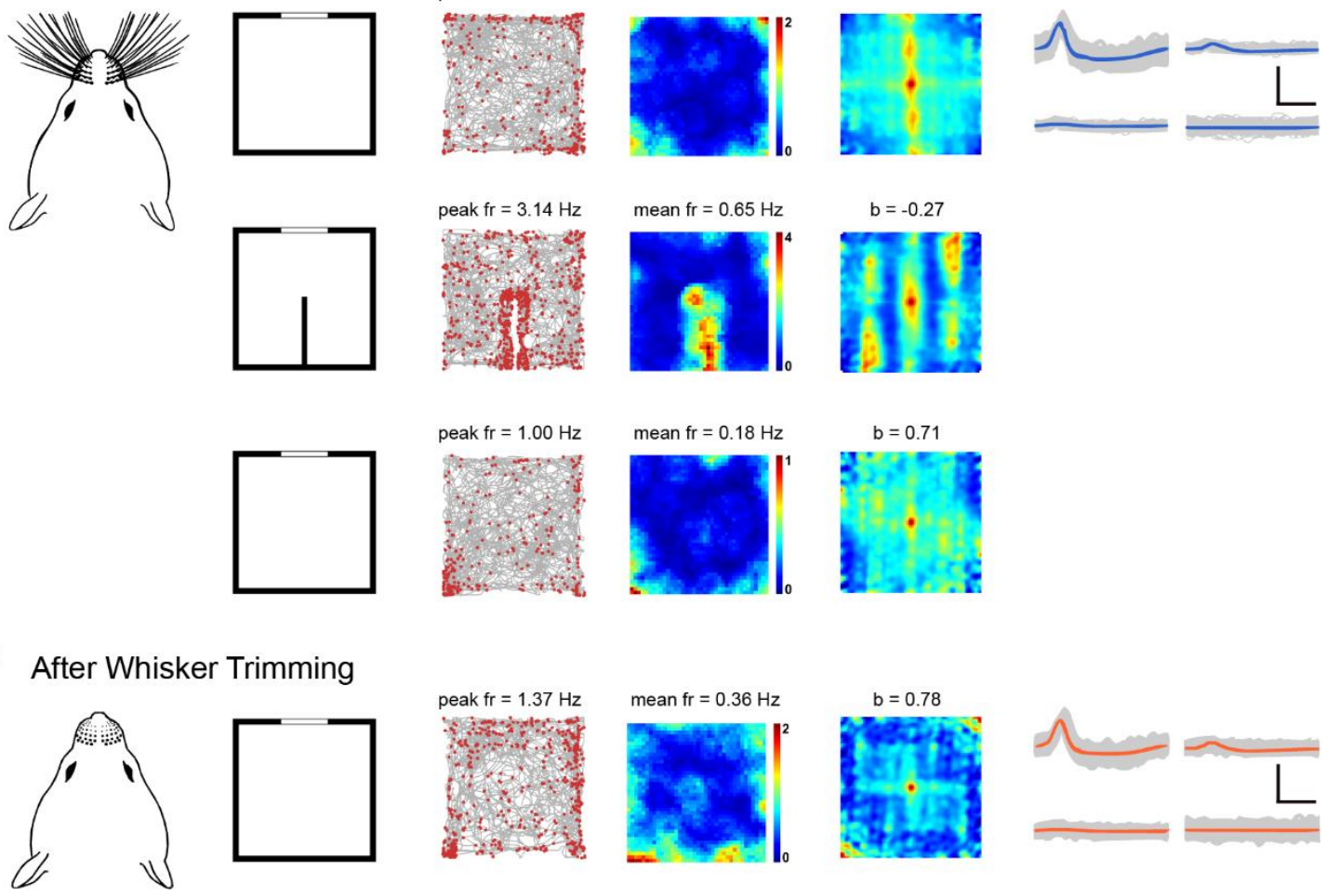
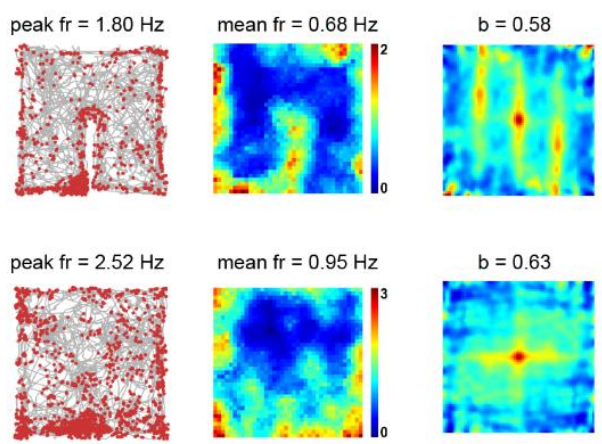

Fig. S33 Preserved spatial firing properties of the same somatosensory border cell before and after whisker trimming.

(a and b) The diagram showing the rat before (a) and after (b) whisker trimming. A representative somatosensory border cell preserves the firing patterns in the square box 
with (top and bottom panels) and without (middle panels) the internal insert before and after whisker trimming. The experimental diagram (left column); trajectory (grey line) with superimposed spike locations (red dots) (middle left column); rate maps (middle right column) and autocorrelation maps (right column) for each recording trial from the same S1 border cell. Firing rate is color-coded with blue indicating minimum firing rate and red indicating maximum firing rate. The scale of the autocorrelation maps is twice that of the spatial firing rate maps. Peak firing rate (fr), mean firing rate (fr) and border score (b) for each recording session are labelled at the top of the panels. Spike waveforms on four electrodes are shown on the right column. Scale bar, $100 \mu \mathrm{V}, 300$ $\mu \mathrm{s}$.

Table S1. Summary of somatosensory spatial cell types recorded in each rat.

\begin{tabular}{|c|c|c|c|c|c|}
\hline & \multicolumn{5}{|c|}{ Somatosensory Spatial Cell Types } \\
\hline Rat Number & $\begin{array}{c}\text { Place } \\
\text { Cells }\end{array}$ & $\begin{array}{c}\text { Head Direction } \\
\text { Cells }\end{array}$ & $\begin{array}{c}\text { Border } \\
\text { Cells }\end{array}$ & $\begin{array}{c}\text { Grid } \\
\text { Cells }\end{array}$ & $\begin{array}{c}\text { Total } \\
\text { Cells }\end{array}$ \\
\hline $1(\mathrm{~S} 1 \mathrm{HL})$ & 22 & 13 & 6 & 12 & 182 \\
\hline $2(\mathrm{~S} 1 \mathrm{HL})$ & 27 & 15 & 14 & 13 & 312 \\
\hline $3(\mathrm{~S} 1 \mathrm{FL})$ & 19 & 9 & 8 & 10 & 353 \\
\hline $4(\mathrm{~S} 1 \mathrm{HL})$ & 20 & 7 & 12 & 9 & 275 \\
\hline $5(\mathrm{~S} 1 \mathrm{Sh})$ & 20 & 6 & 8 & 12 & 263 \\
\hline $6(\mathrm{~S} 1 \mathrm{HL})$ & 35 & 8 & 16 & 7 & 197 \\
\hline $7(\mathrm{~S} 1 \mathrm{HL})$ & 36 & 8 & 12 & 5 & 301 \\
\hline $8(\mathrm{~S} 1 \mathrm{HL})$ & 16 & 14 & 10 & 4 & 142 \\
\hline Total & 195 & 80 & 86 & 72 & 2025 \\
\hline
\end{tabular}

\section{References}

1. Poulter S, Hartley T, Lever C. The Neurobiology of Mammalian Navigation. Current biology : CB 2018; 28:R1023-R1042.

2. Buzsaki G, Moser EI. Memory, navigation and theta rhythm in the hippocampalentorhinal system. Nat Neurosci 2013; 16:130-138.

3. O'Keefe J, Dostrovsky J. The hippocampus as a spatial map. Preliminary evidence from unit activity in the freely-moving rat. Brain Res $1971 ; 34: 171$ 175.

4. Lever C, Burton S, Jeewajee A, O'Keefe J, Burgess N. Boundary vector cells in the subiculum of the hippocampal formation. J Neurosci 2009; 29:9771-9777.

5. Solstad T, Boccara CN, Kropff E, Moser MB, Moser EI. Representation of geometric borders in the entorhinal cortex. Science 2008; 322:1865-1868.

6. Hafting T, Fyhn M, Molden S, Moser MB, Moser EI. Microstructure of a spatial 
map in the entorhinal cortex. Nature 2005; 436:801-806.

7. Sargolini F, Fyhn M, Hafting T et al. Conjunctive representation of position, direction, and velocity in entorhinal cortex. Science 2006; 312:758-762.

8. Taube JS, Muller RU, Ranck JB, Jr. Head-direction cells recorded from the postsubiculum in freely moving rats. I. Description and quantitative analysis. $J$ Neurosci 1990; 10:420-435.

9. Cullen KE, Taube JS. Our sense of direction: progress, controversies and challenges. Nat Neurosci 2017; 20:1465-1473.

10. Town SM, Brimijoin WO, Bizley JK. Egocentric and allocentric representations in auditory cortex. PLoS Biol 2017; 15:e2001878.

11. Fiser A, Mahringer D, Oyibo HK, Petersen AV, Leinweber M, Keller GB. Experience-dependent spatial expectations in mouse visual cortex. Nat Neurosci 2016; 19:1658-1664.

12. Ji D, Wilson MA. Coordinated memory replay in the visual cortex and hippocampus during sleep. Nat Neurosci 2006.

13. Rancz EA, Moya J, Drawitsch F, Brichta AM, Canals S, Margrie TW. Widespread vestibular activation of the rodent cortex. J Neurosci 2015; 35:59265934.

14. Alexander AS, Nitz DA. Spatially Periodic Activation Patterns of Retrosplenial Cortex Encode Route Sub-spaces and Distance Traveled. Current biology : $C B$ 2017; 27:1551-1560 e1554.

15 . Leutgeb S, Mizumori SJ. Context-specific spatial representations by lateral septal cells. Neuroscience 2002; 112:655-663.

16. Wilber AA, Clark BJ, Forster TC, Tatsuno M, McNaughton BL. Interaction of egocentric and world-centered reference frames in the rat posterior parietal cortex. J Neurosci 2014; 34:5431-5446.

17. Boccara CN, Sargolini F, Thoresen VH et al. Grid cells in pre- and parasubiculum. Nat Neurosci 2010; 13:987-994.

18. Weible AP, Rowland DC, Pang R, Kentros C. Neural correlates of novel object and novel location recognition behavior in the mouse anterior cingulate cortex. $J$ Neurophysiol 2009; 102:2055-2068.

19. Mao D, Kandler S, McNaughton BL, Bonin V. Sparse orthogonal population representation of spatial context in the retrosplenial cortex. Nat Commun 2017; 8:243.

20 . Jacob PY, Casali G, Spieser L, Page H, Overington D, Jeffery K. An independent, landmark-dominated head-direction signal in dysgranular retrosplenial cortex. Nat Neurosci 2017; 20:173-175.

21. Jankowski MM, O'Mara SM. Dynamics of place, boundary and object encoding in rat anterior claustrum. Front Behav Neurosci 2015; 9:250.

22. Barry C, Burgess N. To be a Grid Cell: Shuffling procedures for determining "Gridness". bioRxiv 2017.

23. Constantinescu AO, O'Reilly JX, Behrens TEJ. Organizing conceptual knowledge in humans with a gridlike code. Science 2016; 352:1464-1468.

24. Kunz L, Maidenbaum S, Chen D, Wang L, Jacobs J, Axmacher N. Mesoscopic 
Neural Representations in Spatial Navigation. Trends Cogn Sci 2019; 23:615630.

25. Epstein RA, Patai EZ, Julian JB, Spiers HJ. The cognitive map in humans: spatial navigation and beyond. Nat Neurosci 2017; 20:1504-1513.

26. Stangl M, Achtzehn J, Huber K, Dietrich C, Tempelmann C, Wolbers T. Compromised Grid-Cell-like Representations in Old Age as a Key Mechanism to Explain Age-Related Navigational Deficits. Current biology : CB 2018; 28:1108-1115 e1106.

27. Jacobs J, Weidemann CT, Miller JF et al. Direct recordings of grid-like neuronal activity in human spatial navigation. Nat Neurosci 2013; 16:1188-1190.

28. Doeller CF, Barry C, Burgess N. Evidence for grid cells in a human memory network. Nature 2010; 463:657-661.

29 . Maidenbaum S, Miller J, Stein JM, Jacobs J. Grid-like hexadirectional modulation of human entorhinal theta oscillations. Proceedings of the National Academy of Sciences of the United States of America 2018; 115:10798-10803.

30 . Hawkins J, Lewis M, Klukas M, Purdy S, Ahmad S. A Framework for Intelligence and Cortical Function Based on Grid Cells in the Neocortex. Front Neural Circuits 2018; 12:121.

31. Bonnevie T, Dunn B, Fyhn M et al. Grid cells require excitatory drive from the hippocampus. Nat Neurosci 2013; 16:309-317.

32. Hales JB, Schlesiger MI, Leutgeb JK, Squire LR, Leutgeb S, Clark RE. Medial entorhinal cortex lesions only partially disrupt hippocampal place cells and hippocampus-dependent place memory. Cell reports 2014; 9:893-901.

33. Jezek K, Henriksen EJ, Treves A, Moser EI, Moser MB. Theta-paced flickering between place-cell maps in the hippocampus. Nature 2011; 478:246-249.

34 . Muller RU, Kubie JL. The effects of changes in the environment on the spatial firing of hippocampal complex-spike cells. J Neurosci 1987; 7:1951-1968.

35. Zhang S, Manahan-Vaughan D. Spatial Olfactory Learning Contributes to Place Field Formation in the Hippocampus. Cerebral cortex (New York, NY : 1991) 2013.

36. Gener T, Perez-Mendez L, Sanchez-Vives MV. Tactile modulation of hippocampal place fields. Hippocampus 2013.

37 . Brecht M. The Body Model Theory of Somatosensory Cortex. Neuron 2017; 94:985-992.

38. Yin A, Tseng PH, Rajangam S, Lebedev MA, Nicolelis MAL. Place Cell-Like Activity in the Primary Sensorimotor and Premotor Cortex During Monkey Whole-Body Navigation. Sci Rep 2018; 8:9184.

39. McNaughton BL, Battaglia FP, Jensen O, Moser EI, Moser MB. Path integration and the neural basis of the 'cognitive map'. Nat Rev Neurosci 2006; 7:663-678.

40 . Ravassard P, Kees A, Willers B et al. Multisensory control of hippocampal spatiotemporal selectivity. Science 2013; 340:1342-1346.

41. Long X, Zhang SJ. A novel somatosensory spatial navigation system outside the hippocampal formation. bioRxiv 2018; doi: 10.1101/473090.

42. Harris KD, Hirase H, Leinekugel X, Henze DA, Buzsaki G. Temporal 
interaction between single spikes and complex spike bursts in hippocampal pyramidal cells. Neuron 2001; 32:141-149.

43. Zhang SJ, Ye J, Miao C et al. Optogenetic dissection of entorhinal-hippocampal functional connectivity. Science 2013; 340:1232627.

44. Skaggs WE, McNaughton BL, Wilson MA, Barnes CA. Theta phase precession in hippocampal neuronal populations and the compression of temporal sequences. Hippocampus 1996; 6:149-172.

45 . Cacucci F, Lever C, Wills TJ, Burgess N, O'Keefe J. Theta-modulated place-bydirection cells in the hippocampal formation in the rat. $J$ Neurosci 2004; 24:8265-8277.

46. Burgess N, Cacucci F, Lever C, O'Keefe J. Characterizing multiple independent behavioral correlates of cell firing in freely moving animals. Hippocampus 2005; 15:149-153.

47 . Thompson LT, Best PJ. Place cells and silent cells in the hippocampus of freelybehaving rats. J Neurosci 1989; 9:2382-2390.

48 . Jung MW, Wiener SI, McNaughton BL. Comparison of spatial firing characteristics of units in dorsal and ventral hippocampus of the rat. $J$ Neurosci 1994; 14:7347-7356.

49. Rich PD, Liaw HP, Lee AK. Place cells. Large environments reveal the statistical structure governing hippocampal representations. Science 2014; 345:814-817.

50 . Leutgeb S, Leutgeb JK, Barnes CA, Moser EI, McNaughton BL, Moser MB. Independent codes for spatial and episodic memory in hippocampal neuronal ensembles. Science 2005; 309:619-623.

51 . Lever C, Wills T, Cacucci F, Burgess N, O'Keefe J. Long-term plasticity in hippocampal place-cell representation of environmental geometry. Nature 2002; 416:90-94.

52 . Goodridge JP, Dudchenko PA, Worboys KA, Golob EJ, Taube JS. Cue control and head direction cells. Behavioral neuroscience 1998; 112:749-761.

53. Taube JS. Head direction cells recorded in the anterior thalamic nuclei of freely moving rats. J Neurosci 1995; 15:70-86.

54. Stewart S, Jeewajee A, Wills TJ, Burgess N, Lever C. Boundary coding in the rat subiculum. Philos Trans $R$ Soc Lond B Biol Sci 2014; 369:20120514.

55 . Sofroniew NJ, Svoboda K. Whisking. Current biology : CB 2015; 25:R137-140.

56. Chorev E, Preston-Ferrer P, Brecht M. Representation of egomotion in rat's trident and E-row whisker cortices. Nat Neurosci 2016; 19:1367-1373.

57 . Insausti R, Herrero MT, Witter MP. Entorhinal cortex of the rat: cytoarchitectonic subdivisions and the origin and distribution of cortical efferents. Hippocampus 1997; 7:146-183.

58 . Cenquizca LA, Swanson LW. Spatial organization of direct hippocampal field CA1 axonal projections to the rest of the cerebral cortex. Brain Res Rev 2007.

59. Markram H, Muller E, Ramaswamy S et al. Reconstruction and Simulation of Neocortical Microcircuitry. Cell 2015; 163:456-492.

60 . Li CL, Li KC, Wu D et al. Somatosensory neuron types identified by high- 
coverage single-cell RNA-sequencing and functional heterogeneity. Cell Res 2016; 26:83-102.

61. Gaykema RP, Luiten PG, Nyakas C, Traber J. Cortical projection patterns of the medial septum-diagonal band complex. J Comp Neurol 1990; 293:103-124.

62. Bolding KA, Ferbinteanu J, Fox SE, Muller RU. Place cell firing cannot support navigation without intact septal circuits. Hippocampus 2019.

63. Koenig J, Linder AN, Leutgeb JK, Leutgeb S. The spatial periodicity of grid cells is not sustained during reduced theta oscillations. Science 2011; 332:592595.

64. Brandon MP, Bogaard AR, Libby CP, Connerney MA, Gupta K, Hasselmo ME. Reduction of theta rhythm dissociates grid cell spatial periodicity from directional tuning. Science 2011; 332:595-599.

65 . Feldman DE, Brecht M. Map plasticity in somatosensory cortex. Science 2005; 310:810-815.

66. Petersen CCH. Sensorimotor processing in the rodent barrel cortex. Nat Rev Neurosci 2019; 20:533-546.

67. Killackey HP, Sherman SM. Corticothalamic projections from the rat primary somatosensory cortex. J Neurosci 2003; 23:7381-7384.

68. Yamawaki N, Shepherd GM. Synaptic circuit organization of motor corticothalamic neurons. J Neurosci 2015; 35:2293-2307.

69. Schubert D, Staiger JF, Cho N, Kotter R, Zilles K, Luhmann HJ. Layer-specific intracolumnar and transcolumnar functional connectivity of layer $\mathrm{V}$ pyramidal cells in rat barrel cortex. J Neurosci 2001; 21:3580-3592.

70 . Larkum M. A cellular mechanism for cortical associations: an organizing principle for the cerebral cortex. Trends in neurosciences 2013; 36:141-151.

71 . Lefort S, Tomm C, Floyd Sarria JC, Petersen CC. The excitatory neuronal network of the $\mathrm{C} 2$ barrel column in mouse primary somatosensory cortex. Neuron 2009; 61:301-316.

72 . Buzsaki G, Stark E, Berenyi A et al. Tools for probing local circuits: highdensity silicon probes combined with optogenetics. Neuron 2015; 86:92-105.

73. Sieben K, Roder B, Hanganu-Opatz IL. Oscillatory entrainment of primary somatosensory cortex encodes visual control of tactile processing. J Neurosci 2013; 33:5736-5749.

74. Khazipov R, Sirota A, Leinekugel X, Holmes GL, Ben-Ari Y, Buzsaki G. Early motor activity drives spindle bursts in the developing somatosensory cortex. Nature 2004; 432:758-761.

75. Kaas JH. What, if anything, is SI? Organization of first somatosensory area of cortex. Physiological Reviews 1983; 63:206-231.

76. Welker E, Hoogland PV, Van der Loos H. Organization of feedback and feedforward projections of the barrel cortex: a PHA-L study in the mouse. Exp Brain Res 1988; 73:411-435.

77 . Umeda T, Isa T, Nishimura Y. The somatosensory cortex receives information about motor output. Science advances 2019; 5:eaaw5388.

78 . Karadimas SK, Satkunendrarajah K, Laliberte AM et al. Sensory cortical control 
of movement. Nature Neuroscience 2019.

79. Ashe J, Georgopoulos AP. Movement parameters and neural activity in motor cortex and area 5. Cerebral cortex (New York, NY : 1991) 1994; 4:590-600.

80. Moran DW, Schwartz AB. Motor cortical representation of speed and direction during reaching. J Neurophysiol 1999; 82:2676-2692.

81. Mathis A, Mamidanna P, Cury KM et al. DeepLabCut: markerless pose estimation of user-defined body parts with deep learning. Nat Neurosci 2018; 21:1281-1289.

82. Wilson MA, McNaughton BL. Dynamics of the hippocampal ensemble code for space. Science 1993; 261:1055-1058.

83. Paxinos G, Watson C. The rat brain in stereotaxic coordinates. Amsterdam: Elsevier 2007. 